\title{
Dynamics around the double resonance
}

\author{
Chong-Qing Cheng*
}

\begin{abstract}
In this paper, we study time-periodic perturbation of classical systems with two degrees of freedom. A transition chain is established, by passing through small neighborhood of double resonant point, to connect any two cohomology classes corresponding to resonant frequencies. Applying the result to nearly integrable Hamiltonian systems with three degrees of freedom, one obtains a transition chain along which one is able to construct diffusion orbits suggested by Arnold in [A66].
\end{abstract}

AMS 2000 SUBJECT CLASSIFICATIONS: Primary 37J40, 37J50; secondary 49L25.

\section{Introduction and the main result}

In this paper, we consider time-periodic perturbations of the Lagrange system with two degrees of freedom

$$
L_{\epsilon}(x, \dot{x}, t)=\frac{1}{2}\langle A \dot{x}, \dot{x}\rangle+V(x)+\sqrt{\epsilon} R_{\epsilon}(x, \dot{x}, \theta(t)), \quad(x, \theta) \in \mathbb{T}^{2} \times \mathbb{T}
$$

where $A$ is a $2 \times 2$ positive definite matrix of constants, $V \in C^{r}\left(\mathbb{T}^{2}, \mathbb{R}\right)$ with $r \geq 5, R_{\epsilon} \in C^{r-1}\left(\mathbb{T}^{2} \times \mathbb{R}^{2} \times \mathbb{T}, \mathbb{R}\right)$ is a small perturbation, the variable $\theta$ depends on $t$ in two ways, either $\theta=t$ or $\theta(t)=\frac{t}{\varrho \sqrt{\epsilon}}$ where $\varrho$ is a positive constant. As we shall see later, the system in the latter case emerges when one studies Arnold diffusion in nearly integrable Hamiltonian systems with three degrees of freedom around strong double resonance. Regarding $R_{\epsilon}$ as a function of $(\dot{x}, x, \theta)$, we assume that for $\kappa \in\left(0, \frac{1}{2}\right)$

$$
\sqrt{\epsilon}\left\|R_{\epsilon}\right\|_{C^{r-1}} \leq C \epsilon^{\kappa}, \quad \text { for }\left\{|\dot{x}| \leq \epsilon^{-\kappa},(x, \theta) \in \mathbb{T}^{2} \times \mathbb{T}\right\} .
$$

To apply the variational theory, we assume $L_{\epsilon}$ is a Tonelli Lagrangian.

*Supported by some grants from China including NNSF (Grant 11631006), the National Basic Research Program (2013CB834100) and a program PAPD of Jiangsu Province. 
Definition 1.1. Let $M$ be a closed manifold. A $C^{2}$-function $L: T M \times \mathbb{T} \rightarrow \mathbb{R}$ is called Tonelli Lagrangian if it satisfies the following conditions:

Positive Definiteness. For each $(x, t) \in M \times \mathbb{T}$, the Lagrangian function is strictly convex in velocity: the Hessian $\partial_{\dot{x} \dot{x}} L$ is positive definite.

Super-Linear Growth. For each $(x, t) \in M \times \mathbb{T}$, one has $L /\|\dot{x}\| \rightarrow \infty$ as $\|\dot{x}\| \rightarrow \infty$.

COMPLETENEss. All solutions of the Euler-Lagrangian equation are well defined for the whole $t \in \mathbb{R}$.

\subsection{Brief introduction to Mather theory}

It is established for Tonelli Lagrangian. Let $\left\langle\eta_{c}(x), d x\right\rangle$ be a closed 1-form so that $\left[\left\langle\eta_{c}(x), d x\right\rangle\right]=c \in H^{1}(M, \mathbb{R})$, we introduce a Lagrange multiplier $\eta_{c}(x, \dot{x})=\left\langle\eta_{c}(x), \dot{x}\right\rangle$. Abusing the terminology without danger of confusion, we call it closed 1 -form also.

To define the minimal measure, we notice that, $\forall C^{1}$ curve $\gamma: \mathbb{R} \rightarrow M$ with period $k$, there is a unique probability measure $\mu_{\gamma}$ on $T M \times \mathbb{T}$ so that the following holds

$$
\int_{T M \times \mathbb{T}} f d \mu_{\gamma}=\frac{1}{k} \int_{0}^{k} f(d \gamma(s), s) d s
$$

for each $f \in C^{0}(T M \times \mathbb{T}, \mathbb{R})$, where we use the notation $d \gamma=(\gamma, \dot{\gamma})$. Let

$$
\mathfrak{H}^{*}=\left\{\mu_{\gamma} \mid \gamma \in C^{1}(\mathbb{R}, M) \text { is periodic of } k \in \mathbb{Z}^{+}\right\} .
$$

The set $\mathfrak{H}$ of holonomic probability measures is the closure of $\mathfrak{H}^{*}$ in the vector space of continuous linear functionals. Clearly, $\mathfrak{H}$ is convex.

For each $\mu \in \mathfrak{H}$ the action $A_{c}(\mu)$ is defined as follows

$$
A_{c}(\mu)=\int\left(L-\eta_{c}\right) d \mu .
$$

It is proved in [M91, Man] that for each first class $c$ there exists at least one probability measure $\mu_{c}$ minimizing the action over $\mathfrak{H}$

$$
A_{c}\left(\mu_{c}\right)=\inf _{\mu \in \mathfrak{H}} \int\left(L-\eta_{c}\right) d \mu,
$$

which is invariant for the Lagrange flow $\phi_{L}^{t}$, i.e. $\phi_{L}^{t *} \mu_{c}=\mu_{c}$. We call it $c$ minimal measure. Let $\mathfrak{H}_{c} \subset \mathfrak{H}$ be the set of $c$-minimal measures, the Mather 
set $\tilde{\mathcal{M}}(c)$ is defined as

$$
\tilde{\mathcal{M}}(c)=\bigcup_{\mu_{c} \in \mathfrak{H}_{c}} \operatorname{supp} \mu_{c} .
$$

The $\alpha$-function is defined as $\alpha(c)=-A_{c}\left(\mu_{c}\right): H^{1}(M, \mathbb{R}) \rightarrow \mathbb{R}$, it is convex, finite everywhere with super-linear growth. Its Legendre transformation $\beta$ : $H_{1}(M, \mathbb{R}) \rightarrow \mathbb{R}$ is called $\beta$-function

$$
\beta(\omega)=\max _{c}(\langle\omega, c\rangle-\alpha(c))
$$

It is also convex, finite everywhere with super-linear growth (see [M91]).

Notice that $\int \lambda d \mu_{\gamma}=0$ holds for each exact 1-form $\lambda$ and each $\mu_{\gamma} \in \mathfrak{H}^{*}$. For each measure $\mu \in \mathfrak{H}$ one can define its rotation vector $\omega(\mu) \in H_{1}(M, \mathbb{R})$ such that

$$
\langle[\lambda], \omega(\mu)\rangle=\int \lambda d \mu
$$

holds for each closed 1-form $\lambda$ on $M$. According to the definition of holonomic measure, and due to the work in [Man], one has

$$
\beta(\omega)=\inf _{\nu \in \mathfrak{H}_{\omega}} \int L d \nu
$$

where $\mathfrak{H}_{\omega}$ is the set of holonomic probability measures with the rotation vector $\omega$, not necessarily invariant for $\phi_{L}^{t}$.

The Fenchel-Legendre transformation $\mathscr{L}_{\beta}: H_{1}(M, \mathbb{R}) \rightarrow H^{1}(M, \mathbb{R})$ is defined by the following relation

$$
c \in \mathscr{L}_{\beta}(\omega) \Longleftrightarrow \alpha(c)+\beta(\omega)=\langle c, \omega\rangle .
$$

Let

$$
\begin{gathered}
{\left[\left.A_{c}(\gamma)\right|_{\left[t, t^{\prime}\right]}\right]=\int_{t}^{t^{\prime}}\left(L(d \gamma(t), t)-\eta_{c}(d \gamma(t))\right) d t+\alpha(c)\left(t^{\prime}-t\right),} \\
h_{c}\left((x, \tau),\left(x^{\prime}, \tau^{\prime}\right)\right)=\inf _{\substack{\xi \in C^{1}, \xi(\tau)=x \\
\xi\left(\tau^{\prime}\right)=x^{\prime}}}\left[\left.A_{c}(\xi)\right|_{\left[\tau, \tau^{\prime}\right]}\right] \\
F_{c}\left((x, t),\left(x^{\prime}, t^{\prime}\right)\right)=\inf _{\substack{\tau=t \bmod T \\
\tau^{\prime}=t^{\prime} \bmod T}} h_{c}\left((x, \tau),\left(x^{\prime}, \tau^{\prime}\right)\right) .
\end{gathered}
$$

The concept of semi-static curves is introduced in [M93, Man]. A curve $\gamma$ : $\mathbb{R} \rightarrow M$ is called $c$-semi-static if in time- $T$-periodic case we have

$$
\left[\left.A_{c}(\gamma)\right|_{\left[t, t^{\prime}\right]}\right]=F_{c}\left((\gamma(t), t),\left(\gamma\left(t^{\prime}\right), t^{\prime}\right)\right)
$$


A semi-static curve $\gamma \in C^{1}(\mathbb{R}, M)$ is called $c$-static if, in addition, one has the relation

$$
\left[\left.A_{c}(\gamma)\right|_{\left(t, t^{\prime}\right)}\right]=-F_{c}\left(\left(\gamma\left(t^{\prime}\right), t^{\prime}\right),(\gamma(t), t)\right) .
$$

An orbit $X(t)=(\gamma(t), \dot{\gamma}(t), t)$ is called $c$-static (semi-static) if $\gamma$ is $c$-static (semi-static). We call the Mañé set $\tilde{\mathcal{N}}(c)$ the union of $c$-semi-static orbits

$$
\tilde{\mathcal{N}}(c)=\bigcup\{(\gamma(t), \dot{\gamma}(t), t): \gamma \text { is } c \text {-semi static }\}
$$

and call the Aubry set $\tilde{\mathcal{A}}(c)$ the union of $c$-static orbits

$$
\tilde{\mathcal{A}}(c)=\bigcup\{(\gamma(t), \dot{\gamma}(t), t): \gamma \text { is } c \text {-static }\} .
$$

We use $\mathcal{M}(c), \mathcal{A}(c)$ and $\mathcal{N}(c)$ to denote the standard projection of $\tilde{\mathcal{M}}(c)$, $\tilde{\mathcal{A}}(c)$ and $\tilde{\mathcal{N}}(c)$ from $T M \times \mathbb{T}$ to $M \times \mathbb{T}$ respectively. They satisfy the inclusion relation

$$
\tilde{\mathcal{M}}(c) \subseteq \tilde{\mathcal{A}}(c) \subseteq \tilde{\mathcal{N}}(c) .
$$

It is showed in [M91, M93] that the inverse of the projection is Lipschitz when it is restricted to $\mathcal{A}(c)$. By adding subscript $s$ to $\mathcal{N}$, i.e. $\mathcal{N}_{s}$ we denote its time-s-section. This principle also applies to $\tilde{\mathcal{N}}(c), \tilde{\mathcal{A}}(c), \tilde{\mathcal{M}}(c), \mathcal{A}(c)$ and $\mathcal{M}(c)$ to denote their time-s-section respectively. For autonomous systems, these sets are defined without the time component.

A pseudo-metric $d_{c}$ is introduced on Aubry set $\mathcal{A}(c)$ in [M93], its definition relies on the quantity $h_{c}^{\infty}$. Let

$$
h_{c}^{\infty}\left((x, t),\left(x^{\prime}, t^{\prime}\right)\right)=\liminf _{\substack{\tau=t \text { mod } T \\ \tau^{\prime}=t^{\prime} \text { mod } T \\ \tau^{\prime}-\tau \rightarrow \infty}} h_{c}\left((x, \tau),\left(x^{\prime}, \tau^{\prime}\right)\right) .
$$

The pseudo-metric $d_{c}$ on Aubry set is defined as

$$
d_{c}\left((x, t),\left(x^{\prime}, t^{\prime}\right)\right)=h_{c}^{\infty}\left((x, t),\left(x^{\prime}, t^{\prime}\right)\right)+h_{c}^{\infty}\left(\left(x^{\prime}, t^{\prime}\right),(x, t)\right) .
$$

With the pseudo-metric $d_{c}$ one defines equivalence class in Aubry set. The equivalence $(x, t) \sim\left(x^{\prime}, t^{\prime}\right)$ implies $d_{c}\left((x, t),\left(x^{\prime}, t^{\prime}\right)\right)=0$, with which one can define quotient Aubry set $\mathcal{A}(c) / \sim$. Its element is called Aubry class, denoted by $\mathcal{A}_{i}(c)$, its lift to $T M \times \mathbb{T}$ is denoted by $\tilde{\mathcal{A}}_{i}(c)$. Therefore, $\mathcal{A}(c)=\cup_{i \in \Lambda} \mathcal{A}_{i}(c)$, $\tilde{\mathcal{A}}(c)=\cup_{i \in \Lambda} \tilde{\mathcal{A}}_{i}(c)$. It is proved generic in $[\mathrm{BC}]$ that, for system with $n$ degrees of freedom, each $c$-minimal measure contains not more than $n+1$ ergodic components. In this case, each Aubry set contains at most $n+1$ classes. 


\subsection{The main result}

In this paper we consider the conditions that different Aubry sets are connected by orbits of the Euler-Lagrange flow. An orbit is said to connect two invariant sets if its $\alpha$-limit stays in one set while its $\omega$-limit stays in the other. Two Aubry sets are said to be dynamically connected if there is an orbit connecting them.

One way to make sure that two Aubry sets $\tilde{\mathcal{A}}\left(c^{\prime}\right), \tilde{\mathcal{A}}\left(c^{\prime \prime}\right)$ are dynamically connected is to establish a continuous path in the first cohomology space $\Gamma$ : $[0,1] \rightarrow H^{1}(M, \mathbb{R})$ joining $c^{\prime}$ to $c^{\prime \prime}$, namely, $\Gamma(0)=c^{\prime}$ and $\Gamma(1)=c^{\prime \prime}$ and each $s \in[0,1]$ is associated with $\delta_{s}>0$ so that $\tilde{\mathcal{A}}(\Gamma(s))$ is dynamically connected to $\tilde{\mathcal{A}}\left(\Gamma\left(s^{\prime}\right)\right)$ if $\left|s-s^{\prime}\right|<\delta_{s}$. In this case, we say that $\tilde{\mathcal{A}}(\Gamma(s))$ is connected to $\tilde{\mathcal{A}}\left(\Gamma\left(s^{\prime}\right)\right)$ by local connecting orbits. Two types of local connecting orbits are established in [CY1, LC], one looks like heteroclinic orbit, based on the variational version of Arnold's mechanism [A64], and the other one is based on cohomology equivalence developed from that was introduced in [M93], to be defined in Section 3.1. Following Arnold, we call the path a (generalized) transition chain.

Once two cohomology classes $c^{\prime}$ and $c^{\prime \prime}$ are connected by a transition chain, an orbit connecting $\tilde{\mathcal{A}}\left(c^{\prime}\right)$ to $\tilde{\mathcal{A}}\left(c^{\prime \prime}\right)$, which is called a global connecting orbit, is constructed shadowing a sequence of local connecting orbits, as it was done in [CY1, CY2, LC].

Let $\alpha_{\epsilon}, \beta_{\epsilon}$ denote the $\alpha^{-}, \beta$-function for $L_{\epsilon}$ and let $\mathbb{F}_{\epsilon}=\alpha_{\epsilon}^{-1}\left(\min \alpha_{\epsilon}\right)$. Adding a constant to the Lagrangian $L_{\epsilon}$ we assume that $\min \alpha_{\epsilon}=0$. Then we have

Theorem 1.1. There exists a residual set $\mathfrak{V}_{0} \subset C^{r}\left(\mathbb{T}^{2}, \mathbb{R}\right)$ with $r \geq 2$. Each $V \in \mathfrak{V}_{0}$ is associated with some positive numbers $\Delta_{V}, \epsilon_{V}$ such that for any $E \in\left(0, \Delta_{V}\right), \epsilon \in\left[0, \epsilon_{V}\right]$ the circle $\alpha_{\epsilon}^{-1}(E)$ establishes a transition chain (of cohomology equivalence) for the Lagrangian $L_{\epsilon}, \forall c, c^{\prime} \in \alpha_{\epsilon}^{-1}(E)$, the Aubry sets $\mathcal{A}(c)$ and $\mathcal{A}\left(c^{\prime}\right)$ are dynamically connected. These circles make up an annulus $\mathbb{A}_{\epsilon}$ surrounding $\mathbb{F}_{\epsilon}$.

For the second theorem we consider resonant rotation vector. A vector $\omega \in \mathbb{R}^{n}$ is called $k$-resonant if there are exactly $k$ independent integer vectors $\mathbf{k}_{1}, \cdots, \mathbf{k}_{k} \in \mathbb{Z}^{n}$ such that

$$
\left\langle\omega, \mathbf{k}_{j}\right\rangle=0, \quad j=1,2, \cdots, k .
$$

For $n=2$, there is only one double resonant point $\omega=0$. A resonant vector $\omega \neq 0$ is associated with an irreducible class $g \in H_{1}\left(\mathbb{T}^{2}, \mathbb{Z}\right)$ and positive number $\nu$ such that $g=\nu \omega$. Let $\mathbb{C}_{\omega}=\mathbb{C}_{g}=\cup_{\nu>0} \mathscr{L}_{\beta_{\epsilon}}(\nu g) \subset H^{1}\left(\mathbb{T}^{2}, \mathbb{R}\right)$. 
For $r \geq 5$, let $\mathfrak{B}_{\epsilon} \subset C^{r-1}\left(\mathbb{T}^{2} \times \mathbb{R}^{2} \times \mathbb{T}, \mathbb{R}\right)$ be the set of $\sqrt{\epsilon} R_{\epsilon}$ such that the inequality (1.2) holds.

Theorem 1.2. Each resonant rotation vector $\omega \neq 0$ determines an opendense set $\mathfrak{V}_{\omega} \subset C^{r}\left(\mathbb{T}^{2}, \mathbb{R}\right)$ with $r \geq 5$. Each $V \in \mathfrak{V}_{\omega}$ is associated with small positive numbers $\epsilon_{V}$ and $d<\kappa$, for each $\epsilon \in\left(0, \epsilon_{V}\right]$ certain residual set $\mathfrak{R}_{V} \subset \mathfrak{B}_{\epsilon}$ exists such that for each $\sqrt{\epsilon} R_{\epsilon} \in \mathfrak{R}_{V}$, the Lagrangian $L_{\epsilon}$ admits a generalized transition chain $\Gamma_{\omega} \subset \mathbb{C}_{\omega}$ which joins the level set of low energy $\alpha_{\epsilon}^{-1}\left(\epsilon^{d}\right)$ to the level set of high energy $\alpha_{\epsilon}^{-1}\left(\epsilon^{2 \kappa-1}\right)$.

Given a potential $V \in \mathfrak{V}_{0} \cap \mathfrak{V}_{\omega} \cap \mathfrak{V}_{\omega^{\prime}}$, there exists small $\epsilon_{V}>0$ such that $\Delta_{V}>\epsilon_{V}^{d}$. In this case, both $\mathbb{C}_{\omega}$ and $\mathbb{C}_{\omega^{\prime}}$ extend into $\mathbb{A}_{\epsilon}, \tilde{\mathcal{A}}(c)$ is dynamically connected to $\tilde{\mathcal{A}}\left(c^{\prime}\right)$ provided $c \in \mathbb{C}_{\omega}, c^{\prime} \in \mathbb{C}_{\omega^{\prime}}$ and $\alpha(c), \alpha\left(c^{\prime}\right) \leq \epsilon^{-\kappa}$.

The main part of $L_{\epsilon}$ is a classical system with two degrees of freedom

$$
L_{0}(x, \dot{x})=\frac{1}{2}\langle A \dot{x}, \dot{x}\rangle+V(x) .
$$

Let $\alpha_{0}, \beta_{0}$ denote the $\alpha$-, $\beta$-function for $L_{0}$. As $L_{0}$ is autonomous, two Aubry sets $\tilde{\mathcal{A}}(c)$ and $\tilde{\mathcal{A}}\left(c^{\prime}\right)$ are not dynamically connected if $\alpha_{0}(c) \neq \alpha_{0}\left(c^{\prime}\right)$. However, one has

Theorem 1.3. There exists a residual set $\mathfrak{V}_{0} \subset C^{r}\left(\mathbb{T}^{2}, \mathbb{R}\right)$ with $r \geq 2$. Each $V \in \mathfrak{V}_{0}$ is associated with a number $\Delta_{V}>0$ such that for any $E \in\left(0, \Delta_{V}\right)$, the union of Aubry sets $\cup_{c \in \alpha_{0}^{-1}(E)} \tilde{\mathcal{A}}(c)$ is topologically transitive: there exists an orbit of the Euler-Lagrange flow determined by $L_{0}$ such that its $\omega$-limit set contains $\cup_{c \in \alpha_{0}^{-1}(E)} \tilde{\mathcal{A}}(c)$.

Thus, the dynamics in a level set with the energy slightly above Mañés critical value behaves similar to that in Birkhoff instability zones for twist maps. The problem of topological transitivity for geodesic flow on surfaces with negative curvature was illustrated by Birkhoff (see Chapter 8 of [Bir]). However, it is impossible to construct a geodesic flow on 2-torus with negative curvature everywhere, restricted by Gauss-Bonnet formula. Once cohomology equivalence is established among a set of first cohomology classes, such topological transitivity was observed by Mather in [M93].

Throughout the whole paper, to abbreviate the notation, we shall use " $C^{r+}$-generic" to express " $C^{r^{\prime}}$-generic for any integer $r^{\prime} \geq r$ ".

\section{The dynamics around the hyperbolic fixed point}

In this section we restrict ourselves to the classical system

$$
L_{0}=\frac{1}{2}\langle A \dot{x}, \dot{x}\rangle+V(x), \quad(x, \dot{x}) \in T \mathbb{T}^{n}
$$


where $A$ is a positive definite matrix of constants, $\min V=0$. So, one has $\min \alpha_{0}=0$. For $x \in V^{-1}(0)$, the point $(x, 0)$ supports a minimal measure of $L_{0}$ whose rotation vector is 0 . We consider the Fenchel-Legendre duality of 0 ,

$$
\mathbb{F}_{0}=\left\{c \in H^{1}\left(\mathbb{T}^{n}, \mathbb{R}\right): \alpha_{0}(c)=\min \alpha_{0}\right\}=\mathscr{L}_{\beta_{0}}(0) .
$$

We shall show later that this set is a convex disk with full dimensions.

\subsection{Flat of the $\alpha$-function}

A subset is called flat of certain $\alpha$-function if, restricted on this set, the $\alpha$-function is affine, and no longer affine on any set properly containing the set. Since $\alpha$-function is convex with super-linear growth, each flat is a convex and bounded set. Given an $n$-dimensional flat $\mathbb{F}$, a subset $\mathbb{E}$ in $\partial \mathbb{F}$ is called an edge if it is contained in a $(n-1)$-dimensional hyperplane.

Proposition 2.1. Given a class $c \in H^{1}\left(\mathbb{T}^{n}, \mathbb{R}\right)$, if the Mañé set $\mathcal{N}(c)$ lies in a neighborhood of lower dimensional torus $N$ such that $H_{1}\left(\mathbb{T}^{n}, N, \mathbb{Z}\right)=\mathbb{Z}^{k}$ with $k \geq 1$, then there exists a $k$-dimensional flat $\mathbb{F} \subset H^{1}\left(\mathbb{T}^{n}, \mathbb{R}\right)$ such that $c \in \operatorname{int} \mathbb{F}$.

Proof. Let $\mu_{c}, \mu_{c}^{\prime}$ denote the minimal invariant probability measure for the classes $c$ and $c^{\prime}$ respectively. We obtain from the definition of the $\alpha$-function that

$$
\begin{aligned}
& 0=\int L_{0} d \mu_{c^{\prime}}-\left\langle c^{\prime}, \omega\left(\mu_{c^{\prime}}\right)\right\rangle+\alpha\left(c^{\prime}\right) \leq \int L_{0} d \mu_{c^{\prime}}-\left\langle c, \omega\left(\mu_{c^{\prime}}\right)\right\rangle+\alpha(c), \\
& 0=\int L_{0} d \mu_{c}-\left\langle c, \omega\left(\mu_{c}\right)\right\rangle+\alpha(c) \leq \int L_{0} d \mu_{c}-\left\langle c^{\prime}, \omega\left(\mu_{c}\right)\right\rangle+\alpha\left(c^{\prime}\right),
\end{aligned}
$$

where the inequality is obtained since $\mu_{c^{\prime}}$ and $\mu_{c}$ are not necessarily minimal for $c$ and for $c^{\prime}$ respectively. It follows that

$$
\alpha\left(c^{\prime}\right)=\alpha(c) \quad \text { if } \quad\left\langle c-c^{\prime}, \omega\left(\mu_{c^{\prime}}\right)\right\rangle=\left\langle c-c^{\prime}, \omega\left(\mu_{c}\right)\right\rangle=0 .
$$

Since $\mathcal{N}(c) \subset N$ and $H_{1}\left(\mathbb{T}^{n}, N, \mathbb{Z}\right)=\mathbb{Z}^{k}$, the rotation vector $\rho\left(\mu_{c}\right)$ of each ergodic minimal measure $\mu_{c}$ satisfies $k$-resonant conditions, i.e. there are $k$ independent integer vectors $\mathbf{k}_{1}, \cdots, \mathbf{k}_{k}$ such that $\left\langle\mathbf{k}_{i}, \rho(\mu)\right\rangle=0$ holds for each $i=1,2, \cdots, k$.

Because of the upper semi-continuity of Mañé set, one has $\mathcal{N}\left(c^{\prime}\right) \subset N$ if $\left|c^{\prime}-c\right|$ is suitably small. Therefore, there exist a small ball $B_{\delta}(c) \subset H^{1}\left(\mathbb{T}^{n}, \mathbb{R}\right)$ such that for any $c^{\prime} \in B_{\delta}(c)$, the relation $\left\langle\mathbf{k}_{i}, \rho\left(\mu_{c^{\prime}}\right)\right\rangle=0$ holds for each $c^{\prime}$ minimal measure $\mu_{c^{\prime}}$ and for each $i=1,2, \cdots, k$. 
Treating $H^{1}\left(\mathbb{T}^{n}, \mathbb{R}\right)$ as $\mathbb{R}^{n}$, we set $\Pi_{k}=\operatorname{Span}\left\{\mathbf{k}_{1}, \mathbf{k}_{2}, \cdots, \mathbf{k}_{k}\right\}$. It follows from (2.1) that $\alpha(c)=\alpha\left(c^{\prime}\right)$ provided $c^{\prime} \in B_{\delta}(c)$ and $c-c^{\prime} \in \Pi_{k}$. It proves the existence of $k$-dimensional flat.

Proposition 2.2. For the Lagrangian $L_{0}$ we assume the potential $V$ reaches its minimum at one point, then $\mathbb{F}_{0}$ is an n-dimensional disk containing the origin.

Proof. Introducing a coordinate translation $x \rightarrow x+x_{0}$ and adding a constant to $L_{0}$, we can assume that the potential $V$ reaches its minimum at $x=0$ and $L_{0}(0,0)=0$. As $L_{0}(x, \dot{x})>0$ for $(x, \dot{x}) \neq 0$, the minimal measure is uniquely supported on $(x, \dot{x})=0$.

Let us show $x \notin \mathcal{A}(0)$ if $x \neq 0$. If $x \in \mathcal{A}(0)$, there would be a sequence of closed curves $\gamma_{i}:\left[-T_{i}, T_{i}\right] \rightarrow \mathbb{T}^{n}$ such that $\gamma_{i}\left(-T_{i}\right)=\gamma_{i}\left(T_{i}\right)=x$ and $A\left(\gamma_{i}\right) \rightarrow 0$ as $T_{i} \rightarrow \infty$. If $\left|\gamma_{i}\left(T_{i}-\delta\right)\right|=\frac{|x|}{2}$, then it follows from the convexity of kinetic energy in $\dot{x}$ that

$$
\frac{1}{2} \int_{T_{i}-\delta}^{T_{i}}\left\langle A \dot{\gamma}_{i}, \dot{\gamma}_{i}\right\rangle d t \geq \frac{\delta}{2}\left\langle\frac{A}{\delta} \int_{T_{i}-\delta}^{T_{i}} \dot{\gamma}_{i} d t, \frac{1}{\delta} \int_{T_{i}-\delta}^{T_{i}} \dot{\gamma}_{i} d t\right\rangle \geq \frac{\lambda|x|^{2}}{8 \delta}
$$

where $\lambda>0$ is the smallest eigenvalue of $A$. For $x \neq 0$, we set $t_{0}=$ $\min \left\{|x|, \frac{1}{K}\right\}$ with suitably large $K>0$. If $\left|\gamma\left(t^{\prime}\right)-x\right|=\frac{|x|}{2}$ holds for some $t^{\prime} \in\left[T_{i}-t_{0}, T_{i}\right]$, we obtain from the argument just above that

$$
A\left(\gamma_{i}\right)>\frac{1}{2} \int_{T_{i}-t^{\prime}}^{T_{i}}\left\langle A \dot{\gamma}_{i}, \dot{\gamma}_{i}\right\rangle d t \geq \frac{\lambda|x|^{2}}{8} \max \left\{\frac{1}{|x|}, K\right\} .
$$

If there does not exist $t_{0}=\min \left\{|x|, \frac{1}{K}\right\}$ such that $|\gamma(t)-x|<\frac{|x|}{2}$ holds for any $t \in\left[T_{i}-t_{0}, T_{i}\right]$

$$
A\left(\gamma_{i}\right)>\int_{T_{i}-t_{0}}^{T_{i}} V\left(\gamma_{i}\right) d t \geq \min \left\{|x|, \frac{1}{K}\right\} \min _{\left|x^{\prime}\right| \geq \frac{|x|}{2}} V\left(x^{\prime}\right) .
$$

In both cases, $A\left(\gamma_{i}\right)$ is uniformly bounded from below by a positive number as $T_{i} \rightarrow \infty$. It contradicts the assumption that $A\left(\gamma_{i}\right) \rightarrow 0$. Thus, one has $\mathcal{A}(0)=\mathcal{M}(0)$. As the minimal measure is uniquely supported on the fixed point, one has $\mathcal{N}(0)=\mathcal{M}(0)=0$. Applying Proposition 2.1, we complete the proof.

For a Tonelli Lagrangian $L$ and a class in $g \in H_{1}\left(\mathbb{T}^{n}, \mathbb{Z}\right)$, we define

$$
A(g, c)=\liminf _{T_{i} \rightarrow \infty} \inf _{\xi\left(-T_{i}\right)=\xi\left(T_{i}\right) \in \mathcal{M}(c)} \int_{-T_{i}}^{T_{i}} L_{0}(\xi(t), \dot{\xi}(t)) d t-\langle c, g\rangle+2 T_{i} \alpha(c)
$$


which is non-negative. When $N \supset \mathcal{M}(c)$ is open and connected, this definition extends to $g \in H_{1}\left(\mathbb{T}^{n}, N, \mathbb{Z}\right)$, because each closed curve $\xi$ is uniquely associated with a class $[[\xi]]=g \in H_{1}\left(\mathbb{T}^{n}, N, \mathbb{Z}\right)$ also.

Proposition 2.3. If the Lagrangian $L_{0}$ has two degrees of freedom only, $c \notin \mathbb{F}_{0}$, there exists a neighborhood $N$ of a circle such that $\mathcal{M}(c) \subset N$ and $H_{1}\left(\mathbb{T}^{2}, N, \mathbb{Z}\right)=\mathbb{Z}$, then there exists a 1-dimensional flat such that for all $c$ in this flat, the Mather set $\mathcal{M}(c)$ is the same.

Proof. Since $n=2, c \notin \mathbb{F}_{0}, \mathcal{M}(c) \subset N$ and $H_{1}\left(\mathbb{T}^{2}, N, \mathbb{Z}\right)=\mathbb{Z}$, the Mather set consists of periodic orbits. Each closed curve $\gamma_{i}:\left[-T_{i}, T_{i}\right] \rightarrow \mathbb{T}^{2}$ with $\gamma_{i}\left(-T_{i}\right)=\gamma_{i}\left(T_{i}\right) \in \mathcal{M}(c)$ is associated with a class $\left[\gamma_{i}\right] \in H_{1}\left(\mathbb{T}^{2}, \mathbb{Z}\right)$ and a class $\left[\left[\gamma_{i}\right]\right] \in H_{1}\left(\mathbb{T}^{2}, N, \mathbb{Z}\right)$.

Let $g \in H_{1}\left(\mathbb{T}^{2}, N, \mathbb{Z}\right)$ be a generator over $\mathbb{Z}$, we claim $A(g, c)+A(-g, c)>$ 0 . Since the quantity $A(g, c)$ is non-negative, if $A(g, c)+A(-g, c)=0$ there would be two curves $\gamma^{+}, \gamma^{-}$such that $\left[\gamma^{+}\right]=g,\left[\gamma^{-}\right]=-g$, and $\gamma^{+}, \gamma^{-} \in \mathcal{A}(c)$. To see it, let $\gamma_{i}^{+}:\left[-T_{i}, T_{i}\right] \rightarrow \mathbb{T}^{2}$ be such a sequence of minimizers realizing the quantity $A(g, c)$. Let $\left[t_{i}^{-}, t_{i}^{+}\right]$be a connected component of $\left[-T_{i}, T_{i}\right]$ such that $\gamma_{i}^{+}(t) \notin N$ if $t \in\left(t_{i}^{-}, t_{i}^{+}\right)$and $\gamma_{i}^{+}\left(t_{i}^{ \pm}\right) \in \partial N$. There must be such a connected component such that $\left[\left[\left.\gamma_{i}^{+}\right|_{\left[t_{i}^{-}, t_{i}^{+}\right]}\right]\right]=g$. If $\mathcal{M}(c)$ does not touch the boundary of $N, t_{i}^{+}-t_{i}^{-}$is uniformly bounded. So, there exists a number $t_{0}$ such that $\left[-t_{0}, t_{0}\right] \supset\left[t_{i}^{-}, t_{i}^{+}\right]$, by a time-translation, for all large $T_{i}$. As each $\gamma_{i}^{+}$is a minimizer, $\dot{\gamma}_{i}^{+}$is uniformly bounded. As it solves the Lagrange equation $\ddot{x}=\left(\partial_{\dot{x}, \dot{x}} L\right)^{-1}\left(\partial_{x} L-\dot{x} \partial_{\dot{x}, x} L\right)$ and $L$ is positive definite, we find the set $\left\{\left.\gamma_{i}^{+}\right|_{\left[-t_{0}, t_{0}\right]}\right\}$ is compact in $C^{1}$-topology. Let $t_{0} \rightarrow \infty$. By the diagonal extraction argument some subsequence of $\left\{\gamma_{i}\right\}$ converges $C^{1}$ uniformly, when they are restricted on any compact set containing $\left[-t_{0}, t_{0}\right]$, to a $C^{1}$-curve $\gamma^{+}: \mathbb{R} \rightarrow M$. Clearly, $\left[\left[\gamma^{+}\right]\right]=g$. Because $A(g, c)=0$, this curve must be in the Aubry set $\mathcal{A}(c)$. In the same way, we see that the $\gamma^{-}$ also lies in $\mathcal{A}(c)$ and $\left[\left[\gamma^{-}\right]\right]=-g$.

However, it is impossible since it would violate Lipschitz graph property of Aubry set, for the curve $\gamma^{-}$would intersect the curve $\gamma^{+}$somewhere since the configuration space is 2-torus. If $A(-g, c)=0$, one has $A(g, c)=a>0$.

Let $\mu_{c}$ be a minimal measure for $c$, which is supported on periodic orbit in this case. Given a class $c^{\prime} \in H^{1}\left(\mathbb{T}^{2}, \mathbb{R}\right)$ such that $\left\langle c^{\prime}-c, \omega\left(\mu_{c}\right)\right\rangle=0$, one has

$$
0 \leq \int L_{0} d \mu_{c}-\left\langle c^{\prime}, \omega\left(\mu_{c}\right)\right\rangle+\alpha\left(c^{\prime}\right)=\alpha\left(c^{\prime}\right)-\alpha(c) .
$$

As the $\alpha$-function is convex, the rotation vector $\omega\left(\mu_{c^{\prime}}\right)$ stays in the set of sub-derivative of $\alpha$ at $c^{\prime}$. So, one has $\left\langle\omega\left(\mu_{c^{\prime}}\right), c-c^{\prime}\right\rangle \leq \alpha(c)-\alpha\left(c^{\prime}\right) \leq 0$. 
Let $c^{\prime}$ be a class such that $c^{\prime}-c=\nu g$ with small $\nu>0$. Treating $c, c^{\prime}$ and $g$ as vectors in $\mathbb{R}^{2}$, we claim $\left\langle\omega\left(\mu_{c^{\prime}}\right), c^{\prime}-c\right\rangle=0$. If $\left\langle\omega\left(\mu_{c^{\prime}}\right), c^{\prime}-c\right\rangle>0$, there exists a curve $\gamma$ lying in $\mathcal{M}(c)$ as well as two numbers $t, \delta>0$ such that $B_{\delta}(\gamma(t)) \subset N$. We set

$$
T(\gamma, t, \delta)=\min \left\{t^{\prime}>0: d\left(\gamma(t), \gamma\left(t+t^{\prime}\right)\right)<\delta,\left[\left[\left.\gamma\right|_{\left[t, t+t^{\prime}\right]}\right]\right]=g\right\}
$$

It follows from the upper semi-continuity of Mañé set in the first cohomology class that $T(\gamma, t, \delta) \rightarrow \infty$ as $c^{\prime} \rightarrow c$ and $\delta \rightarrow 0$. Since $\gamma$ lies in $\mathcal{M}\left(c^{\prime}\right)$

$$
\int_{t}^{t+T(\gamma, t, \delta)}\left(L_{0}(\gamma(s), \dot{\gamma}(s))-\left\langle c^{\prime}, \dot{\gamma}(s)\right\rangle+\alpha\left(c^{\prime}\right)\right) d s \leq C \delta .
$$

On the other hand, because of $A(g, c)=a>0, \exists \epsilon \rightarrow 0$ as $T(\gamma, t, \delta) \rightarrow \infty$

$$
\int_{t}^{t+T(\gamma, t, \delta)}\left(L_{0}(\gamma(s), \dot{\gamma}(s))-\langle c, \dot{\gamma}(s)\rangle+\alpha(c)\right) d s \geq a-C \delta-\epsilon .
$$

Putting these two inequalities together, letting $\bar{\gamma}$ denote the lift of $\gamma$ to the universal covering space $\mathbb{R}^{2}$, one has

$$
T(\gamma, t, \delta)\left(\alpha(c)-\alpha\left(c^{\prime}\right)\right)+\left\langle c^{\prime}-c, \bar{\gamma}(t+T(\gamma, t, \delta))-\bar{\gamma}(t)\right\rangle \geq a-2 C \delta-\epsilon
$$

But it is absurd as $\alpha(c)-\alpha\left(c^{\prime}\right) \leq 0,\left[\left[\left.\gamma\right|_{[t, t+T(\gamma, t, \delta)]}\right]\right]=g$ and $c^{\prime}-c=\nu g$ with small $\nu$.

Since $\left\langle c^{\prime}-c, \rho\left(\mu_{c^{\prime}}\right)\right\rangle=\left\langle c^{\prime}-c, \rho\left(\mu_{c}\right)\right\rangle=0$, we obtain from (2.1) that $\alpha\left(c^{\prime}\right)=\alpha(c)$.

For a $k$-dimensional flat $\mathbb{F}$, to define its interior and the boundary, we treat it as a set in $\mathbb{R}^{k}$. Then, they follow from the standard definitions, denoted by int $\mathbb{F}$ and $\partial \mathbb{F}$ respectively.

Proposition 2.4 ([Mas]). Let $\mathbb{F}$ be a flat of $\alpha$-function, then $\mathcal{A}(c)=\mathcal{A}\left(c^{\prime}\right)$ if $c, c^{\prime} \in \operatorname{int} \mathbb{F}$ and $\mathcal{A}(c) \supseteq \mathcal{A}\left(c^{\prime}\right)$ if $c^{\prime} \in \partial \mathbb{F}$ and $c \in \operatorname{int} \mathbb{F}$.

\subsection{Around the flat of the minimum}

In this subsection we will restrict ourselves to the classical system with two degrees of freedom and investigate the structure of the Mather sets and the Mañé sets for each $c \in \partial \mathbb{F}_{0}$. It is $C^{2+}$-generic to assume

(H1): the potential $V$ attains its minimum at one point only, where the Hessian matrix $\partial_{x}^{2} V$ is positive definite and the matrix $J \operatorname{diag}\left\{A^{-1}, \partial_{x}^{2} V\right\}$ has 4 different real eigenvalues $-\lambda_{2}<-\lambda_{1}<0<\lambda_{1}<\lambda_{2}$. 
Up to a translation of coordinates and addition of constant to $V$, one can assume $\min V=0$ which is attained at $x=0$.

In virtue of Proposition 2.2, the flat $\mathbb{F}_{0}$ is a 2 -dimensional disk when the hypothesis (H1) is assumed. Now, let us study the shape of the disk $\mathbb{F}_{0}$ and divide the boundary of $\mathbb{F}_{0}$ into two parts $\partial \mathbb{F}_{0}=\partial^{*} \mathbb{F}_{0} \cup\left(\partial \mathbb{F}_{0} \backslash \partial^{*} \mathbb{F}_{0}\right)$, where

$$
\partial^{*} \mathbb{F}_{0}=\left\{c \in \partial \mathbb{F}_{0}: \mathcal{M}(c) \backslash\{x=0\} \neq \varnothing\right\} .
$$

Clearly $0 \in \operatorname{int} \mathbb{F}_{0}, \mathcal{N}(c)=\{0\}$ is a singleton $\forall c \in \operatorname{int} \mathbb{F}_{0}$. Given $c \in \partial^{*} \mathbb{F}_{0}$, we use $\mu_{c}$ to denote the minimal measure which is not supported on the fixed point. Since the configuration space is a 2-torus, all minimal measures, except the one supported on the fixed point, share the same rotation vector, denoted by $\omega\left(\mu_{c}\right)$.

It is possible that the set $\partial^{*} \mathbb{F}_{0}$ is non-empty. Here is an example:

$$
L=\frac{1}{2} \dot{x}_{1}^{2}+\frac{\nu^{2}}{2} \dot{x}_{2}^{2}+V(x)
$$

where $|\nu| \neq 1$, the potential satisfies the following conditions: $x=0$ is the minimal point of $V$ only, there exist two numbers $d>d^{\prime}>0$ such that

1. $V=d^{\prime}+\left(x_{2}-a\right)^{2}$ as it is restricted in a neighborhood of circle $x_{2}=a$ with $a \neq 0 \bmod 1$;

2. $V=P\left(\sqrt{x_{1}^{2}+x_{2}^{2}}\right)$ for $|x| \leq 2 \delta$, where $P$ is a bump function $P(r)=$ $B e^{-\frac{1}{\delta-|r-\delta|}}$ for $r \in(0,2 \delta)$. By choosing suitably large $B>0$, for any closed curve $\gamma:[-T, T] \rightarrow \mathbb{T}^{2}$ passing through the origin with $[\gamma] \neq 0$ one has

$$
\int_{-T}^{T} L(\gamma(s), \dot{\gamma}(s)) d s \geq d .
$$

In this case, we find that $\partial \mathbb{F}_{0} \cap\left\{c_{2}=0\right\}=\left\{c_{1}= \pm \sqrt{2 d^{\prime}}\right\}$. Indeed,

$$
L \pm c_{1} \dot{x}_{1}=\frac{1}{2}\left(\dot{x}_{1} \pm c_{1}\right)^{2}+\frac{\nu^{2}}{2} \dot{x}_{2}^{2}+V(x)-\frac{1}{2} c_{1}^{2},
$$

the $\mathcal{M}(c)=\{0\} \cup\left\{\left(x_{1,0} \mp \sqrt{2 d^{\prime}} t, a\right): t \in \mathbb{R}\right\}$ for $c=\left( \pm \sqrt{2 d^{\prime}}, 0\right)$. The set $\partial^{*} \mathbb{F}_{0}$ is closed in $\partial \mathbb{F}_{0}$. If it is non-empty, the existence of infinitely many homoclinic orbits has been proved in [Zhe, Zm2]. These orbits are associated with different homological classes $\left\{g_{i}\right\}$, each of these orbits is minimal when it is lifted to certain finite covering manifold. When $\left|g_{i}\right| \rightarrow \infty$, these orbits are getting closer and closer to the support of that minimal measure not supported on the fixed point. 


\subsubsection{The structure of $\partial F_{0}$ and the phase space correspondence.} A homoclinic orbit $(\gamma, \dot{\gamma})$ is called minimal if the action along the curve $\gamma$ is not large than the action along any other curve $\zeta$ homoclinic to the point provided $[\zeta]=[\gamma]$. In this case, $\gamma$ is called minimal homoclinic curve.

If $c \in \partial \mathbb{F}_{0} \backslash \partial^{*} \mathbb{F}_{0}$, the Mañé set is the same as the Aubry set, consisting of the fixed point $(x, \dot{x})=0$ and some minimal homoclinic orbit to the fixed point, it can not be the fixed point alone, since one has

Lemma 2.1. Let $\mathbb{F}_{0}$ be a 2-dimensional flat, the Mather set be a singleton for each class in the interior of $\mathbb{F}_{0}$, and let $\mathbb{E}_{i}$ be an edge of $\mathbb{F}_{0}$, then $\mathcal{A}\left(c^{\prime}\right)=$ $\mathcal{A}(c)$ if $c^{\prime}, c \subset \operatorname{int} \mathbb{E}_{i}$ and

$$
\mathcal{A}\left(c^{\prime}\right) \supsetneq \mathcal{A}(c)
$$

holds for $c^{\prime} \in \partial \mathbb{F}_{0}\left(\partial \mathbb{E}_{i}\right)$ and $c \in \operatorname{int} \mathbb{F}\left(\operatorname{int} \mathbb{E}_{i}\right)$ respectively.

Proof. Recall the proof of Proposition 2.2, we see that $\mathcal{N}(0)$ is a singleton. Applying Proposition 2.4, the Mañé set is that singleton $\forall c \in \operatorname{int} \mathbb{F}_{0}$. For a class $c \in \partial \mathbb{F}_{0} \backslash \partial^{*} \mathbb{F}_{0}$, the Aubry set $\mathcal{A}(c)$ contains at least one minimal homoclinic curve with non-zero first homology. Otherwise, for a class $c^{\prime} \notin \mathbb{F}_{0}$ very close to $c$, the homology of the Mañé set is trivial, the same as that for $c$. It is guaranteed by the upper semi-continuity of Mañe set in cohomology class. It follows that $\left\langle c, \omega\left(\mu_{c}\right)\right\rangle=\left\langle c^{\prime}, \rho\left(\mu_{c}^{\prime}\right)\right\rangle=0$ and

$$
-\alpha_{0}\left(c^{\prime}\right)=A\left(\mu_{c^{\prime}}\right)-\left\langle c^{\prime}, \rho\left(\mu_{c}^{\prime}\right)\right\rangle \geq A\left(\mu_{c}\right)=-\alpha_{0}(c) .
$$

However, one has $\alpha_{0}\left(c^{\prime}\right)>\alpha_{0}(c)$ for $c^{\prime} \notin \mathbb{F}_{0}$. The contradiction verifies our claim. When $c^{\prime} \in \partial^{*} \mathbb{F}_{0}$, the certain $c^{\prime}$-minimal measure $\mu_{c^{\prime}}$ exists with $\rho\left(\mu_{c^{\prime}}\right) \neq 0$. In both cases, we have $\mathcal{A}\left(c^{\prime}\right) \supsetneq \mathcal{A}(c)$ if $c \in \operatorname{int} \mathbb{F}_{0}$ and $c^{\prime} \in \partial \mathbb{F}_{0}$.

To show $\mathcal{A}\left(c^{\prime}\right)=\mathcal{A}(c)$ for $c^{\prime}, c \subset \operatorname{int} \mathbb{E}_{i}$, one follows the way of [Mas]. As $c^{\prime}, c \subset \operatorname{int} \mathbb{E}_{i}, \exists c^{\prime \prime} \in \mathbb{E}_{i}, \lambda \in(0,1)$ such that $c^{\prime}=(1-\lambda) c+\lambda c^{\prime \prime}$. For any small $\epsilon>0, x \in \mathcal{A}(c), \exists$ a curve $\gamma:[0, T] \rightarrow M$ with $\gamma(0)=\gamma(T)=x$ and

$$
\begin{aligned}
\epsilon> & \int_{0}^{T}\left(L(\gamma(t), \dot{\gamma}(t))-\left\langle c^{\prime}, \dot{\gamma}(t)\right\rangle\right) d t \\
= & (1-\lambda) \int_{0}^{T}(L(\gamma(t), \dot{\gamma}(t))-\langle c, \dot{\gamma}(t)\rangle) d t \\
& +\lambda \int_{0}^{T}\left(L(\gamma(t), \dot{\gamma}(t))-\left\langle c^{\prime \prime}, \dot{\gamma}(t)\right\rangle\right) d t .
\end{aligned}
$$

Since $\epsilon>0$ can be arbitrarily small, one has $x \in \mathcal{A}\left(c^{\prime}\right)$.

Let $\mathbb{E}_{i}$ be an edge, then it follows from Proposition 2.4 that either int $\mathbb{E}_{i} \cap$ $\partial^{*} \mathbb{F}_{0}=\varnothing$ or $\operatorname{int} \mathbb{E}_{i} \subset \partial^{*} \mathbb{F}_{0}$. In the former case, the Aubry set contains 
homoclinic orbits which share the same homological class. Indeed, given two homoclinic orbits $\left(\gamma_{1}, \dot{\gamma}_{1}\right)$ and $\left(\gamma_{2}, \dot{\gamma}_{2}\right)$ associated with class $\left[\gamma_{1}\right]$ and $\left[\gamma_{2}\right]$ respectively, one has $\left[A_{c}\left(\gamma_{j}\right)\right]=0$ for $j=1,2$ and for $c=c^{\prime}, c^{\prime \prime} \in \operatorname{int} \mathbb{E}_{i}$ (see $(2.2))$. It follows that $\left\langle c^{\prime}-c^{\prime \prime},\left[\gamma_{j}\right]\right\rangle=0$ holds for $c, c^{\prime} \in \operatorname{int} \mathbb{E}_{i}$ and $j=1,2$. As each curve $\gamma_{j}$ is minimal and the configuration space is a 2-torus, $\left[\gamma_{j}\right]$ has to be irreducible, otherwise, the curve will intersect itself. Therefore, one has $\left[\gamma_{1}\right]=\left[\gamma_{2}\right]$, denoted by $g\left(\mathbb{E}_{i}\right)$. In latter case, all ergodic minimal measures, except the one supported on the fixed point, also share the same rotation vector.

Let $c^{\prime} \in \partial \mathbb{E}_{i}$ and $c \in \operatorname{int} \mathbb{E}_{i}$, one chooses $c^{*} \in \partial \mathbb{F}_{0} \backslash \mathbb{E}_{i}$ arbitrarily close to $c^{\prime}$. As the straight line connecting $c$ to $c^{*}$ passes through the interior of $\mathbb{F}_{0}$, we obtain from Proposition 2.4 that $\mathcal{A}(c) \cap \mathcal{A}\left(c^{*}\right)=\mathcal{A}\left(c_{0}\right)$ with $c_{0} \in \operatorname{int} \mathbb{F}_{0}$. If $\tilde{\mathcal{A}}\left(c^{*}\right) \backslash \tilde{\mathcal{A}}\left(c_{0}\right)$ contains a homoclinic orbit $(\zeta, \dot{\zeta})$, it follows from the following equation

$$
0=\int\left(L_{0}(d \zeta(t))-\left\langle c^{*}, \dot{\zeta}\right\rangle\right) d t=\int\left(L_{0}(d \zeta(t))-\langle c, \dot{\zeta}\rangle\right) d t+\left\langle c-c^{*},[\zeta]\right\rangle
$$

that $\left\langle c-c^{*},[\zeta]\right\rangle \neq 0$ holds. We claim $[\zeta] \neq g\left(\mathbb{E}_{i}\right)$. Let us assume the contrary and consider the case that $\mathcal{A}(c)$ contains a homoclinic orbit $(\gamma, \dot{\gamma})$. Because $\min \alpha_{0}=0$, one has

$$
\int_{-\infty}^{\infty} L_{0}(\zeta, \dot{\zeta}) d t-\left\langle c^{*},[\zeta]\right\rangle=0, \quad \int_{-\infty}^{\infty} L_{0}(\gamma, \dot{\gamma}) d t-\left\langle c, g\left(\mathbb{E}_{i}\right)\right\rangle=0 .
$$

Since the class $c^{*}$ is not on the straight line containing $\mathbb{E}_{i}$, we have $\left\langle c^{*}-\right.$ $\left.c, g\left(\mathbb{E}_{i}\right)\right\rangle \neq 0$. If $\left\langle c^{*}-c, g\left(\mathbb{E}_{i}\right)\right\rangle>0$ one would have

$$
\int_{-\infty}^{\infty} L_{0}(\gamma, \dot{\gamma}) d t-\left\langle c^{*},[\gamma]\right\rangle=\int_{-\infty}^{\infty} L_{0}(\gamma, \dot{\gamma}) d t-\langle c,[\gamma]\rangle-\left\langle c^{*}-c, g\left(\mathbb{E}_{i}\right)\right\rangle<0
$$

If $[\zeta]=g\left(\mathbb{E}_{i}\right)$ and $\left\langle c^{*}-c, g\left(\mathbb{E}_{i}\right)\right\rangle<0$ we would have

$$
\int_{-\infty}^{\infty} L_{0}(\zeta, \dot{\zeta}) d t-\langle c,[\zeta]\rangle=\int_{-\infty}^{\infty} L_{0}(\zeta, \dot{\zeta}) d t-\left\langle c^{*},[\zeta]\right\rangle+\left\langle c^{*}-c, g\left(\mathbb{E}_{i}\right)\right\rangle<0 .
$$

Both cases are absurd since $\alpha_{0}(c)=\alpha_{0}\left(c^{*}\right)=0$, the left hand side of above inequalities can not be negative. Since $[\zeta] \neq g\left(\mathbb{E}_{i}\right)$, some $x^{*} \in \mathcal{A}\left(c^{*}\right)$ remains far away from $\mathcal{A}(c)$. Let $c^{*} \rightarrow c^{\prime}$, the accumulation point of these points does not fall into $\mathcal{A}(c)$, therefore it implies $\mathcal{A}\left(c^{\prime}\right) \supsetneq \mathcal{A}(c)$. The proof is similar if $\zeta$ and $\gamma$ are curves lying in the Mather set. 
Let $G_{c} \subset H_{1}\left(\mathbb{T}^{2}, \mathbb{Z}\right)$ be a set that $g \in G_{c}$ if there is a minimal homoclinic orbit $(\gamma, \dot{\gamma})$ in $\tilde{\mathcal{A}}(c)$ with $[\gamma]=g$. We say that there are $k$-types of minimal homoclinic orbits in $\tilde{\mathcal{A}}(c)$ if $G_{c}$ contains exactly $k$ elements. Given an edge $\mathbb{E}_{i}$, we define $G_{\mathbb{E}_{i}}=G_{c}$ for each $c \in \operatorname{int} \mathbb{E}_{i}$ since all classes in int $\mathbb{E}_{i}$ share the same Aubry set (Proposition 2.4).

Theorem 2.1. Let $\mathbb{F}_{0}=\alpha_{0}^{-1}\left(\min \alpha_{0}\right)$ be a two dimensional flat, $\mathcal{M}\left(c_{0}\right)$ be a singleton for $c_{0} \in$ int $\mathbb{F}_{0}$. Let $\mathbb{E}_{i}$ denote an edge of $\mathbb{F}_{0}$ (not a point), then

1, either $\mathbb{E}_{i} \cap \partial^{*} \mathbb{F}_{0}=\varnothing$ or $\mathbb{E}_{i} \subset \partial^{*} \mathbb{F}_{0}$;

2, if $\mathbb{E}_{i} \cap \partial^{*} \mathbb{F}_{0}=\varnothing$, then $G_{\mathbb{E}_{i}}$ contains exactly one element, if $\mathbb{E}_{i} \subset \partial^{*} \mathbb{F}_{0}$, all curves in $\mathcal{M}\left(\mathbb{E}_{i}\right) \backslash\{0\}$ have the same rotation vector;

3, if $c \in \partial \mathbb{E}_{i}$ and $c \notin \partial^{*} \mathbb{F}_{0}$ then $G_{c}$ contains exactly two elements;

4, if $\mathbb{E}_{i}, \mathbb{E}_{j} \subset \partial^{*} \mathbb{F}_{0}$, then either $\mathbb{E}_{i}=\mathbb{E}_{j}$, or $\mathbb{E}_{i}$ is disjointed to $\mathbb{E}_{j}$;

5, if $\mathbb{E}_{i} \subset \partial^{*} \mathbb{F}_{0}, \mathcal{M}(c)=\mathcal{M}\left(c^{\prime}\right)$ holds for $c \in \partial \mathbb{E}_{i}$ and $c^{\prime} \in$ int $\mathbb{E}_{i}$;

6 , if $\mu_{c}$ is an ergodic c-minimal measure for $c \in \partial^{*} \mathbb{F}_{0}$ and $\omega\left(\mu_{c}\right)$ is irrational, then the class $c$ is an extremal point of $\mathbb{F}_{0}$;

7 , if $c \in \partial \mathbb{F}_{0} \backslash \partial^{*} \mathbb{F}_{0}$ and $\tilde{\mathcal{A}}(c)$ consists of the fixed point and a homoclinic orbit $(\gamma, \dot{\gamma})$ only, then $c$ is located in the interior of certain edge $\mathbb{E}_{i}$;

8 , each edge in $\partial \mathbb{F}_{0} \backslash \partial^{*} \mathbb{F}_{0}$ is joined by two edges in $\partial \mathbb{F}_{0} \backslash \partial^{*} \mathbb{F}_{0}$.

Proof. It follows from Lemma 2.1 that $\mathcal{A}(c)=\mathcal{A}\left(c^{\prime}\right)$ if $c, c^{\prime} \in \operatorname{int} \mathbb{E}_{i}$. So, if the item 1 is not true and $\operatorname{int} \mathbb{E}_{i} \cap \partial^{*} \mathbb{F}_{0}=\varnothing$, there would exist an invariant measure $\mu_{c}$ for the class $c \in \partial \mathbb{E}_{i}$, not supported on the singleton which minimizes the action

$$
\int L_{0} d \mu_{c}-\left\langle\rho\left(\mu_{c}\right), c\right\rangle=-\alpha_{0}(c)=0,
$$

but not minimizes the $c^{\prime}$-action for $c^{\prime} \in \operatorname{int} \mathbb{E}_{i}$. As the configuration space is $\mathbb{T}^{2}$, the Lipschitz graph property of Aubry set will be violated if the rotation vector of the measure $\omega\left(\mu_{c}\right)$ is not parallel to $g \in G_{\mathbb{E}_{i}}$. So, $\left\langle\omega\left(\mu_{c}\right), c-c^{\prime}\right\rangle=0$ holds for $c^{\prime} \in \operatorname{int} \mathbb{E}_{i}$. It follows that $\mu_{c}$ also minimizes the action for $c^{\prime} \in \operatorname{int} \mathbb{E}_{i}$. This leads to a contradiction. If int $\mathbb{E}_{i} \subset \partial^{*} \mathbb{F}_{0}$, then the formula (2.4) holds for each $c \in \operatorname{int} \mathbb{E}_{i}$. Let $\operatorname{int}_{\mathbb{E}_{i}} \ni c^{\prime} \rightarrow \partial \mathbb{E}_{i},(2.4)$ holds for $c \in \partial \mathbb{E}_{i}$, namely, the whole edge is contained in $\partial^{*} \mathbb{F}_{0}$.

The item 2 follows from the fact that $\left\langle c-c^{\prime},[\gamma]\right\rangle=0$ holds for any $c, c^{\prime} \in \operatorname{int} \mathbb{E}_{i}$ and any $\gamma \in \mathcal{A}(c)$. The item 3 follows from that $\mathcal{A}(c) \nsupseteq \mathcal{A}\left(c^{\prime}\right)$ if $c^{\prime} \in \operatorname{int} \mathbb{E}_{i}$, the Lipschitz property would be violated if $G_{c}$ contains three or more elements.

If the item 4 were not true, for the cohomology class in $\mathbb{E}_{i} \cap \mathbb{E}_{j}$ the Mather set would contain two closed circles with different homology, but it 
violates the Lipschitz graph property of Aubry set. With the same reason we have the item 5 .

If the item 6 were not true, the class $c$ lies in the interior of an edge $\mathbb{E}$, whose normal direction is $\omega\left(\mu_{c}\right)$. Let $c^{\prime} \in \partial \mathbb{E}$, it follows from Lemma 2.1 that there exists some point $x \in \mathcal{A}\left(c^{\prime}\right) \backslash \mathcal{A}(c)$. There exists a sequence of closed curves $\gamma_{i}^{\prime}:\left[-T_{i}, T_{i}\right] \rightarrow \mathbb{T}^{2}$ such that $\gamma_{i}^{\prime}\left(-T_{i}\right)=\gamma_{i}^{\prime}\left(T_{i}\right)$ and $\left[A_{c^{\prime}}\left(\gamma_{i}^{\prime}\right)\right] \rightarrow 0$ as $T_{i} \rightarrow \infty$. Regarding $\left[\gamma_{i}^{\prime}\right]$ as a vector in $\mathbb{R}^{2}$, it is impossible that $\left[\gamma_{i}^{\prime}\right] /\left\|\left[\gamma_{i}^{\prime}\right]\right\| \nrightarrow$ $\omega\left(\mu_{c}\right) /\left\|\omega\left(\mu_{c}\right)\right\|$ because it will violate the Lipschitz graph property as $\omega\left(\mu_{c}\right)$ is irrational. The fact $\left[\gamma_{i}^{\prime}\right] /\left\|\left[\gamma_{i}^{\prime}\right]\right\| \rightarrow \omega\left(\mu_{c}\right) /\left\|\omega\left(\mu_{c}\right)\right\|$ implies $x \in \mathcal{A}(c)$.

By the condition of the item 7 , one has $\tilde{\mathcal{N}}(c)=\tilde{\mathcal{A}}(c)$ for $c \in \partial \mathbb{F}_{0} \backslash \partial^{*} \mathbb{F}_{0}$. Due to the upper semi-continuity of Mañé set in the first cohomology class, $\tilde{\mathcal{N}}\left(c^{\prime}\right)$ lies in a small neighborhood of the orbit $(\gamma, \dot{\gamma})$ provided $c^{\prime}$ is close to $c$. If such $c^{\prime}$ lies on $\partial \mathbb{F}_{0}$, the Aubry set for $c^{\prime}$ must contain some homoclinic or periodic orbit $\left(\gamma^{\prime}, \dot{\gamma}^{\prime}\right)$ which entirely stays in a small neighborhood of $\gamma$. Because of the topology of $\mathbb{T}^{2}$, one has $[\gamma]=\left[\gamma^{\prime}\right]$. Let $A_{0}\left(\gamma^{\prime}\right)=\int L_{0}\left(\gamma^{\prime}, \dot{\gamma}^{\prime}\right) d t$. If $\left\langle c-c^{\prime},[\gamma]\right\rangle>0$, one obtains from $\alpha_{0}\left(c^{\prime}\right)=0$ that

$$
A_{0}\left(\gamma^{\prime}\right)-\left\langle c,\left[\gamma^{\prime}\right]\right\rangle=A_{0}\left(\gamma^{\prime}\right)-\left\langle c^{\prime},\left[\gamma^{\prime}\right]\right\rangle+\left\langle c^{\prime}-c,\left[\gamma^{\prime}\right]\right\rangle<0,
$$

but it is absurd because the $c$-action along any closed curve is non-negative. It is also obviously impossible that $\left\langle c-c^{\prime},[\gamma]\right\rangle<0$. Thus, one obtains from

$$
A_{0}\left(\gamma^{\prime}\right)-\left\langle c,\left[\gamma^{\prime}\right]\right\rangle=A_{0}\left(\gamma^{\prime}\right)-\left\langle c^{\prime},\left[\gamma^{\prime}\right]\right\rangle=0 .
$$

that $\gamma^{\prime}=\gamma$, namely, $c$ stays in the interior of certain edge $\mathbb{E}$ and $\left\langle c-c^{\prime},[\gamma]\right\rangle=$ 0 holds $c, c^{\prime} \in \mathbb{E}$.

Let us prove the last one. Given an edge $\mathbb{E} \subset \partial \mathbb{F}_{0} \backslash \partial^{*} \mathbb{F}_{0}$. There exists a homoclinic curve $\gamma$ which is $c$-minimal for all $c \in \mathbb{E}$. For $c^{\prime} \in \partial \mathbb{E}$, the Aubry set $\mathcal{A}\left(c^{\prime}\right)$ must contain, besides the curve $\gamma$, another homoclinic curve $\gamma^{\prime}$ with $\left[\gamma^{\prime}\right] \neq[\gamma]$. We identify $H^{1}\left(\mathbb{T}^{2}, \mathbb{R}\right)$ with $\mathbb{R}^{2}$ where we choose $\mathbb{L}$ to be a straight line passing through the point $c^{\prime}$ and orthogonal to the vector $\left[\gamma^{\prime}\right]$. Since $A_{0}\left(\gamma^{\prime}\right)-\left\langle c^{\prime \prime},\left[\gamma^{\prime}\right]\right\rangle=A_{0}\left(\gamma^{\prime}\right)-\left\langle c^{\prime},\left[\gamma^{\prime}\right]\right\rangle=0$ holds for each $c^{\prime \prime} \in \mathbb{L}$ and the Aubry set contains the fixed point only when the class stays in the interior, any point on $\mathbb{L}$ does not lie in the interior of $\mathbb{F}_{0}$. If there exists a class $c^{\prime \prime} \in \mathbb{L}$ other than $c^{\prime}$ so that $\alpha\left(c^{\prime \prime}\right)=0$ we assert $c^{\prime \prime} \in \partial \mathbb{F} \backslash \partial^{*} \mathbb{F}_{0}$. Otherwise, some $c^{\prime \prime}$-minimal measure $\mu_{c^{\prime \prime}}$ would exist with $\omega\left(\mu_{c^{\prime \prime}}\right) \neq 0, \mu_{c^{\prime \prime}}$ is supported on a periodic orbit $\left(\gamma^{\prime \prime}, \dot{\gamma}^{\prime \prime}\right)$ with $\left[\gamma^{\prime \prime}\right]=\left[\gamma^{\prime}\right]$. Therefore, $\gamma^{\prime \prime}$ also lies in $\mathcal{A}\left(c^{\prime}\right)$. However, it is absurd because it violates the Lipschitz property of Aubry set because $\left[\gamma^{\prime \prime}\right] \neq[\gamma]$. Therefore, if $\exists c^{\prime \prime} \in \mathbb{L} \neq c^{\prime}$ with $\alpha\left(c^{\prime \prime}\right)=0$, the edge containing $c^{\prime}$ to $c^{\prime \prime}$ is contained in $\partial \mathbb{F} \backslash \partial^{*} \mathbb{F}_{0}$, joint to $\mathbb{E}$ at $c^{\prime}$. 
Let us show that there does exist such point on $\mathbb{L}$. The line $\mathbb{L}$ divide the plane $H^{1}\left(\mathbb{T}^{2}, \mathbb{R}\right)$ into two parts. Let $c_{i} \in \partial \mathbb{F}_{0} \backslash \mathbb{E} \rightarrow c^{\prime}$ be a sequence of classes on $\partial \mathbb{F}_{0}$ which approach $c^{\prime}$ from outside of $\mathbb{E}$. If no point on $\mathbb{L}$ belongs to $\mathbb{F}_{0}$ other than $c^{\prime}$, these points and the edge $\mathbb{E}$ stay in the same side of $\mathbb{L}$. As $\alpha_{0}$ is a convex function and $\mathbb{F}_{0}$ is convex set touching the line $\mathbb{L}$ at one point $c^{\prime}$, each vertex $v_{i}$ of the sub-derivative of $\alpha_{0}$ at $c_{i}$ has the expression of $v_{i}=-\nu_{i}[\gamma]+\nu_{i}^{\prime}\left[\gamma^{\prime}\right]$ where both $\nu_{i}$ and $\nu_{i}^{\prime}$ are non-negative. If $\nu_{i}>0$, there would be a segment of certain $c_{i}$-minimal curve falls in the vicinity of $\gamma$ due to the upper semi-continuity $c \rightarrow \mathcal{N}(c)$, but moves in an opposite direction of $\dot{\gamma}$ because of $\nu_{i}>0$. It contradicts the upper semi-continuity $c \rightarrow \tilde{\mathcal{N}}(c)$. Therefore, the rotation vector of each ergodic $c_{i}$-minimal measure $\mu_{i}$ takes the form of $\nu_{i}^{\prime}\left[\gamma^{\prime}\right]$, or $\tilde{\mathcal{A}}\left(c_{i}\right)$ contains a homoclinic orbit $\left(\gamma^{\prime \prime}, \dot{\gamma}^{\prime \prime}\right)$ such that $\left[\gamma^{\prime \prime}\right]=\left[\gamma^{\prime}\right]$. It follows from the convexity of $\mathbb{F}_{0}$ that $c_{i} \in \mathbb{L}$. It contradicts the assumption.

Let $\mathbb{E}_{i} \subset \partial \mathbb{F}_{0}^{*}$ be a edge. When $c$ moves along $\mathbb{E}_{i}$ to its boundary, what emerges in the Aubry set is just heteroclinic orbit connecting the periodic orbit and the fixed point. It is the consequence of upper semi-continuity of Mañé set on the first cohomology class. When $c^{\prime}$ approaches $c \in \partial \mathbb{E}_{i}$ from outside of $\mathbb{E}_{i}, \mathcal{N}\left(c^{\prime}\right)$ falls into the vicinity of $\mathcal{N}(c)$. It would contradicts the condition $\omega\left(c^{\prime}\right) \neq \omega(c)$ if $\tilde{\mathcal{N}}(c)$ consists of the periodic orbit and the fixed point, so it contains some orbit connecting the periodic orbit and the fixed point which is approached by a sequence of $c_{i}$-static orbit with $c_{i} \notin \mathbb{E}_{i} \rightarrow c^{\prime}$. Consequently, the curve is $c^{\prime}$-static, see Fig. 1.

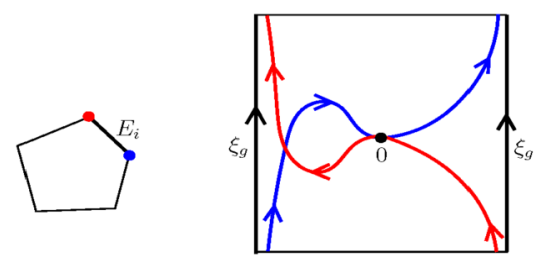

Figure 1: $\mathcal{M}\left(\mathbb{E}_{i}\right)=\{0\} \cup\left\{\xi_{g}\right\}$. The blue curve is in $\mathcal{A}(c)$ for $c$ at one end point of $\mathbb{E}_{i}$, the red curve is in $\mathcal{A}\left(c^{\prime}\right)$ for $c^{\prime}$ at another end point.

Lemma 2.2. Let $\mathbb{E}, \mathbb{E}^{\prime} \subset \partial \mathbb{F}_{0} \backslash \partial^{*} \mathbb{F}_{0}$ be two adjacent edges and assume $c \in$ $\mathbb{E} \cap \mathbb{E}^{\prime}$. If $(m, n)=g \in G_{\mathbb{E}}$ and $\left(m^{\prime}, n^{\prime}\right)=g^{\prime} \in G_{\mathbb{E}^{\prime}}$, then $m^{\prime} n-m n^{\prime}= \pm 1$.

Proof. The Aubry set $\tilde{\mathcal{A}}(c)$ contains homoclinic orbits with the class $(m, n)$ as well as $\left(m^{\prime}, n^{\prime}\right)$, both are irreducible. Guaranteed by the Lipschitz graph property, these two curves intersect each other only at the fixed point. In the 
universal covering space $\mathbb{R}^{2}$, each curve in the lift of the homoclinic curves are determined by the equation

$$
m x_{1}+n x_{2}=k, \quad m^{\prime} x_{1}+n^{\prime} x_{2}=k^{\prime} .
$$

The solution of the equations corresponds to the intersection point which are lattice points in $\mathbb{Z}^{2}$ for any $\left(k, k^{\prime}\right) \in \mathbb{Z}^{2}$. To guarantee this property, the necessary and sufficient condition is $m n^{\prime}-m^{\prime} n= \pm 1$.

We ask if the set $\partial \mathbb{F}_{0} \backslash \partial^{*} \mathbb{F}_{0}$ can be empty. If not, it follows from Theorem 2.1 that the $\alpha$-function will be not differentiable. For the topic of differentiability, see [MS].

\subsubsection{Dynamics in a neighborhood of $\mathbb{F}_{0}$ with $\alpha_{0}(c) \geq \min \alpha_{0}$.} To proceed the demonstration, we assume further conditions on the classical system. The Lagrangian $L_{0}$ induces a Hamiltonian $G=\frac{1}{2}\left\langle A^{-1} y, y\right\rangle-V(x)$. The point $(x, y)=0$ is a fixed point of the Hamiltonian flow, which is hyperbolic as $A$ is positive definite. Recall (H1), the matrix $J \operatorname{diag}\left\{A^{-1}, \partial_{x}^{2} V(0)\right\}$ has 4 different real eigenvalues $\pm \lambda_{1}, \pm \lambda_{2}$. If we denote by $\Lambda_{i}^{+}=\left(\Lambda_{i, x}, \Lambda_{i, y}\right)$ the eigenvector for the eigenvalue $\lambda_{i}(i=1,2)$, where $\Lambda_{i, x}$ and $\Lambda_{i, y}$ are for the $x$ - and $y$-coordinate respectively, then the eigenvector for $-\lambda_{i}$ will be $\Lambda_{i}^{-}=\left(\Lambda_{i, y},-\Lambda_{i, y}\right)$.

Let $z=(x, y)$. The fixed point $z=0$ has its stable and unstable manifold, denoted by $W^{+}$and $W^{-}$respectively. Each point $z \in W^{+} \cap W^{-} \backslash\{0\}$ lies on certain orbit homoclinic to the point $z=0$. As each homoclinic orbit entirely stays in the energy level set $H^{-1}(0)$, along the orbit their intersection can not be transversal in the standard definition, but can be transversal when they are restricted to the energy level, namely

$$
T_{z} W^{-} \oplus T_{z} W^{+}=T_{z} H^{-1}(0)
$$

holds for each $z$ is on homoclinic orbit. Without causing danger of confusion, we call the intersection transversal also. Since $H_{1}\left(\mathbb{T}^{2}, \mathbb{Z}\right)$ contains countably many elements, it is also a $C^{2+}$-generic condition that

(H2): For each $g \in H_{1}\left(\mathbb{T}^{2}, \mathbb{Z}\right)$, there is at most one minimal homoclinic curve $\gamma$ such that $[\gamma]=g$, the stable manifold intersects the unstable manifold transversally along each minimal homoclinic orbit. Each minimal homoclinic orbit approaches to the fixed point in the direction $\Lambda_{1}: \dot{\gamma}(t) /\|\dot{\gamma}(t)\| \rightarrow \Lambda_{x 1}$ as $t \rightarrow \pm \infty$.

Next, we fix an irreducible class $g \in H_{1}\left(\mathbb{T}^{2}, \mathbb{Z}\right) \backslash\{0\}$ and study the structure of $\mathcal{M}\left(c_{\nu}\right)$ with $c_{\nu} \in \mathscr{L}_{\beta_{0}}(\nu g)$ and $\alpha_{0}\left(c_{\nu}\right)>\min \alpha_{0}$. As the configuration 
space is $\mathbb{T}^{2}$, the rotation vector is rational, each ergodic $c$-minimal measure is supported on a periodic orbit. To specify the topological information of a minimal periodic orbit (curve), we call it $\nu g$-minimal if some irreducible class $g \in H_{1}\left(\mathbb{T}^{2}, \mathbb{Z}\right)$ and $\nu>0$ exist such that it is $c$-minimal $\forall c \in \mathscr{L}_{\beta}(\nu g)$. An orbit is called $\nu g$-periodic if it is merely $\frac{1}{\nu}$-periodic with the homology type $g$, no minimal property is implied.

Let $\nu>0$ decrease such that $\alpha_{0}\left(c_{\nu}\right) \downarrow \min \alpha_{0}$. There are two possibilities. Either $\mathscr{L}_{\beta_{0}}(\nu g) \notin \partial \mathbb{F}_{0}$ for any $\nu>0$, or $\exists \nu_{0}>0$ such that $\mathscr{L}_{\beta_{0}}\left(\nu_{0} g\right) \in \partial \mathbb{F}_{0}$, see the example of (2.3). In the latter case, $\mathscr{L}_{\beta_{0}}\left(\nu_{0} g\right) \in \partial^{*} \mathbb{F}_{0}$. For the latter case, one has

Proposition 2.5. Assume some $\nu_{0}>0$ exists such that $\mathscr{L}_{\beta_{0}}\left(\nu_{0} g\right) \in \partial^{*} \mathbb{F}_{0}$. For $\nu_{1}>\nu_{0}$, there is an open-dense set $\mathfrak{V} \subset C^{r}\left(\mathbb{T}^{2}, \mathbb{R}\right)$ with $r \geq 5$ such that for each $V \in \mathfrak{V}, c \in \mathscr{L}_{\beta_{0}}(\nu g)$ with $\nu \in\left(\nu_{0}, \nu_{1}\right]$, the Mather set consists of hyperbolic $\nu$-minimal periodic orbits. Indeed, there are finitely many $\nu_{i} \in$ $\left(\nu_{0}, \nu_{1}\right)$ such that the set contains exactly two periodic orbits for $c \in \mathscr{L}_{\beta_{0}}\left(\nu_{i} g\right)$ and contains exactly one periodic orbit if $\nu \neq \nu_{i}$ and $\nu \in\left(\nu_{0}, \nu_{1}\right]$.

Proof. We apply a result in [CZ2]. For a Tonelli Lagrangian $L \in C^{r}\left(T \mathbb{T}^{2}, \mathbb{R}\right)$, let $\alpha^{\prime}, \beta^{\prime}$ denote the $\alpha$-, $\beta$-function of $L+V$ respectively, one has

Theorem $2.2([\mathrm{CZ} 2])$. For a class $g \in H_{1}\left(\mathbb{T}^{2}, \mathbb{Z}\right) \backslash\{0\}$ and a closed interval $\left[E_{a}, E_{b}\right]$ with $E_{a}>\min \alpha$, there exists an open-dense set $\mathfrak{V} \subset C^{r}\left(\mathbb{T}^{2}, \mathbb{R}\right)$ with $r \geq 5$ such that for each $V \in \mathfrak{V}$ it holds simultaneously for all $E \in\left[E_{a}, E_{b}\right]$ that the Mather set $\tilde{\mathcal{M}}(c)$ for $L+V$ consists of hyperbolic $\nu$ g-periodic orbits if $c \in \alpha^{\prime-1}(E) \cap \cup_{\nu>0} \mathscr{L}_{\beta^{\prime}}(\nu g)$. Indeed, there are finitely many $E_{i} \in\left[E_{a}, E_{b}\right]$ such that the Mather set contains exactly two periodic orbits for $E=E_{i}$ and contains one periodic orbit only if $E \neq E_{i}$.

It is clearly an open-dense condition on $V$ in $C^{r}(r \geq 2)$ that the Mather set for $c \in \mathscr{L}_{\beta}\left(\nu_{0} g\right)$ is constituted by the fixed point and a hyperbolic periodic orbit $\left(\xi_{g}, \dot{\xi}_{g}\right)$. Choosing a perturbation $V \rightarrow V-\delta V$ such that $\delta V \geq 0$ and $\operatorname{supp} \delta V$ is a small disk containing the point $x=0$, disjoint with the periodic curve $\xi_{g}$. Clearly, for $\nu>\nu_{0}$ with small $\left|\nu-\nu_{0}\right|$, the support of $\mathscr{L}_{\beta_{0}}(\nu g)$-minimal measure of $L_{0}$ does not touch $\operatorname{supp} \delta V$. It follows that the support of $\mathscr{L}_{\beta_{0}}(\nu g)$-minimal measure of $L_{0}-\delta V$ is the same as that for $L_{0}$. Let $\alpha^{\prime}$ be the $\alpha$-function of $L_{0}-\delta V$, then $\min \alpha^{\prime}<\alpha^{\prime}\left(\mathscr{L}_{\beta_{0}}\left(\nu_{0} g\right)\right)$. We then finish the proof by applying Theorem 2.2.

Theorem 2.2 is proved by variational method. Let $\xi$ be a closed curve on the torus $\mathbb{T}^{2}$ such that $H_{1}\left(\mathbb{T}^{2}, \mathbb{Z}\right)$ is spanned by $\{g,[\xi]\}$. Define

$$
F(x, E)=\min _{\substack{\gamma(0)=\gamma\left(T_{E}\right)=x \\[\gamma]=g}} \int_{0}^{T_{E}} L_{0}(\gamma(t), \dot{\gamma}(t)) d t
$$


where the time $T_{E}$ is set so that the minimal curve $\gamma$ generates an orbit $\left(\gamma, \partial_{\dot{x}} L_{0}(\gamma, \dot{\gamma})\right)$ lying on the energy level set $G^{-1}(E)$. If $x_{E}$ is a minimal point of $F(\cdot, E)$, the minimal curve passing through the point $x_{E}$ generates a periodic orbit. The orbit is hyperbolic if and only if the minimal point is non-degenerate. So, the problem becomes to check whether it holds simultaneously for all $E \in\left[E_{a}, E_{b}\right]$ that all minimal points of $F(\cdot, E)$ is nondegenerate. The Lipschitz dependence of $F$ in $E$ plays key role, one is unable to get the non-degeneracy if some Hölder instead of Lipschitz continuity is assumed [Zm3].

By Proposition 2.5, there exist finitely many pieces of normally hyperbolic invariant cylinders (NHICs) made up by $\nu g$-minimal periodic orbits where $\nu$ ranges over the set $\left[\nu_{0}, \nu_{1}\right]$. Next, let us consider the former case that $\mathscr{L}_{\beta_{0}}(\nu g) \rightarrow \partial \mathbb{F}_{0}$ as $\nu \downarrow 0$.

Proposition 2.6. We assume the hypotheses $(\mathbf{H 1}, \mathbf{2})$ for $L_{0}$, for some $g \in$ $H_{1}\left(\mathbb{T}^{2}, \mathbb{Z}\right)$ one has $\alpha_{0}\left(\mathscr{L}_{\beta_{0}}(\nu g)\right)>0$ for all $\nu>0$. Then, there exist $\nu_{0}>0$ and finitely many NHICs which are foliated by $\nu$ g-periodic orbits $\nu \in\left(0, \nu_{0}\right]$, each $\nu$ g-minimal periodic orbit lies on one of the cylinders.

Proof. Since $L_{0}$ is autonomous and the configuration space is $\mathbb{T}^{2}, \mathscr{L}_{\beta_{0}}(\nu g)$ is either an interval or a point, each ergodic $c$-minimal measure is supported on certain $\nu g$-minimal periodic orbit if $c \in \mathscr{L}_{\beta_{0}}(\nu g)$ and $\nu>0$. It follows that some set $\mathbb{E}_{g} \subset \partial \mathbb{F}_{0} \backslash \partial^{*} \mathbb{F}_{0}$ exists such that $\mathscr{L}_{\beta_{0}}(\nu g) \rightarrow \mathbb{E}_{g}$ as $\nu \rightarrow 0$ in the sense of Hausdorff. Therefore, for $c \in \mathbb{E}_{g}$, the Aubry set is the same as the Mañé set, consists of homoclinic orbits and the hyperbolic fixed point. Due to the upper semi-continuity of Mañe set in the first cohomology, the Kuratowski upper limit set of the sequence of periodic orbits consists of homoclinic orbit(s) staying in $\tilde{\mathcal{A}}(c)$ for $c \in \mathbb{E}_{g}$.

According to Theorem 2.1 (the items 7 and 8), the set $\partial \mathbb{F}_{0} \backslash \partial^{*} \mathbb{F}_{0}$ consists of edges $\left\{\mathbb{E}_{i}\right\}$ only. Under the hypothesis (H2), the Aubry set consists of one homoclinic curve for $c \in \operatorname{int} \mathbb{E}_{i}$, and consists of exactly two homoclinic curves $\gamma_{g_{i}}$ and $\gamma_{g_{i+1}}$ if $c \in \mathbb{E}_{i} \cap \mathbb{E}_{i+1}$ (a vertex). In latter case, $\operatorname{det}\left(g_{i}, g_{i+1}\right)= \pm 1$ if $g_{i}$ and $g_{i+1}$ are treated as vectors in $\mathbb{Z}^{2}$, see Lemma 2.2. It is impossible that $\mathcal{A}(c)$ contains more than two homoclinics with different homology types, which violates the Lipschitz property of Aubry set, one can refer to [M11] for more details.

Let us study a general case $g=k_{i} g_{i}+k_{i+1} g_{i+1}$. Through the Legendre transformation $y=A \dot{x}$, one obtains from the Lagrangian $L_{0}$ the Hamiltonian $G=\frac{1}{2}\left\langle A^{-1} y, y\right\rangle-V(x)$. Each $c$-minimal orbit $(x(t), \dot{x}(t))$ lies in the energy level set $\left\{G^{-1}\left(\alpha_{0}(c)\right)\right\}$ in the sense that $G(x(t), y(t))=\alpha_{0}(c)$. Denote by $z_{i}(t)=\left(x_{i}(t), y_{i}(t)\right)$ the homoclinic orbit such that $\left[x_{i}\right]=g_{i}$ and $\left[x_{i+1}\right]=$ 
$g_{i+1}$. Because of $(\mathbf{H 2})$, one has

$$
\lim _{t \rightarrow \pm \infty} \frac{\dot{z}_{i}(t)}{\left|\dot{z}_{i}(t)\right|}=\lim _{t \rightarrow \pm \infty} \frac{\dot{z}_{i+1}(t)}{\left|\dot{z}_{i+1}(t)\right|} .
$$

To proceed the proof, we quote a result (Lemma 2.2 in [C15]):

Lemma 2.3 ([C15]). Assume the hypotheses $(\mathbf{H 1}, \mathbf{2})$. For a class $g=k_{i} g_{i}+$ $k_{i+1} g_{i+1}\left(k_{i}, k_{i+1}>0\right)$, we assume that, as $\nu \downarrow 0$, there is a $\nu$-periodic orbit $z_{\nu}(t)$ approaching the minimal homoclinic orbits $z_{i}$ and $z_{i+1}$. Then, some $\nu_{0}>0$ exists such that for each $\nu \in\left(0, \nu_{0}\right]$, there is a two-dimensional disk $\Sigma_{\nu} \subset G^{-1}\left(\alpha_{0}\left(\mathscr{L}_{\beta_{0}}(\nu g)\right)\right)$ intersecting the orbit $z_{\nu}(t)$ transversally. Restricted $\Sigma_{\nu}$, the Hamiltonian flow $\Phi_{G}^{t}$ induces a Poincaré return map $\Phi_{\nu}: \Sigma_{\nu} \rightarrow \Sigma_{\nu}$, and there exists some $\lambda>1, C>1$ independent of $\nu \leq \nu_{0}$ such that

$$
\begin{aligned}
& \left\|D \Phi_{\nu}\left(z_{\nu, 0}\right) v^{-}\right\| \geq C E_{\nu}^{-\lambda}\left\|v^{-}\right\|, \quad \forall v^{-} \in T_{z_{\nu, 0}} W_{\nu}^{-} \\
& \left\|D \Phi_{\nu}\left(z_{\nu, 0}\right) v^{+}\right\| \leq C^{-1} E_{\nu}^{\lambda}\left\|v^{-}\right\|, \quad \forall v^{+} \in T_{z_{\nu, 0}} W_{\nu}^{+},
\end{aligned}
$$

where $E_{\nu}=\alpha_{0}\left(\mathscr{L}_{\beta_{0}}(\nu g)\right), z_{\nu, 0}$ is the point where the periodic orbit intersects $\Sigma_{\nu}$, and $W_{\nu}^{ \pm}$denotes the stable (unstable) manifold of the periodic orbit.

As each $\nu g$-minimal periodic orbit falls into the vicinity of the homoclinic orbits, the Lemma guarantees the hyperbolicity when it is restricted on the energy level of 3 -dimension $G^{-1}\left(\alpha_{0}\left(\mathscr{L}_{\beta_{0}}(\nu g)\right)\right)$. By the estimate on the normal hyperbolicity and the implicit function theorem, each $\nu g$-minimal periodic orbit has its continuation of a continuous family of hyperbolic $\nu g$ periodic orbits as $\nu$ ranges over the interval $\left(0, \nu_{0}\right]$. We do not study the uniqueness of the cylinder, it is enough for our approach.

To study what is the shape of the set $\mathbb{C}_{g}=\cup_{\nu>0} \mathscr{L}_{\beta_{0}}(\nu g)$, letting $E_{a}$ in Theorem 2.2 be a sequence of numbers $E_{i} \downarrow \min \alpha$, each $E_{i}$ is associated with an open-dense set $\mathfrak{V}_{i} \subset C^{r}\left(\mathbb{T}^{2}, \mathbb{R}\right)$ with $r \geq 5$ such that Theorem 2.2 holds. Taking the intersection of $\mathfrak{V}_{i}$ one obtains a $C^{r}$-residual property that each $\nu g$-minimal orbit is hyperbolic. It follows from Proposition 2.3 that $\mathscr{L}_{\beta_{0}}(\nu g)$ is an interval provided $\nu>0$, namely, $\mathbb{C}_{g}$ is foliated by intervals $I_{\nu, g}$. Therefore, the set looks like a channel. The length of $I_{\nu, g}$ may or may not approach zero as $\nu \downarrow 0$, depending on whether there is some edge $\mathbb{E}_{i} \subset$ $\partial \mathbb{F}_{0} \backslash \partial^{*} \mathbb{F}_{0}$ such that $g \in G_{\mathbb{E}_{i}}$.

If $g \in G_{\mathbb{E}_{i}}$ holds for some $\mathbb{E}_{i} \subset \partial \mathbb{F}_{0} \backslash \partial^{*} \mathbb{F}_{0}$, the length of $\mathscr{L}_{\beta_{0}}(\nu g)$ does not shrink to zero as $\nu \downarrow 0$ and the Hausdorff distance between two sets $\mathbb{E}_{i}$ and $\mathscr{L}_{\beta_{0}}(\nu g)$ approaches zero as $\nu \downarrow 0$, forced by the upper semi-continuity of $c \rightarrow \tilde{\mathcal{N}}(c)$. 
If there does not exist such an edge, then there are a pair of adjacent edges $\mathbb{E}_{i}, \mathbb{E}_{i+1}$ and positive integers $k_{i}, k_{i+1}$ such that $g=k_{i} g_{i}+k_{i+1} g_{i+1}$, the channels $\mathbb{C}_{g_{i}}$ and $\mathbb{C}_{g_{i+1}}$ join at a vertex of $\mathbb{F}_{0}$. The interval $\mathscr{L}_{\beta_{0}}(\nu g)$ is located between $\mathbb{C}_{i}$ and $\mathbb{C}_{i+1}$. When $\nu \downarrow 0$, it shrinks to the vertex where $\mathbb{E}_{i}$ is joined to $\mathbb{E}_{i+1}$. Therefore, the channel $\mathbb{C}_{g}$ appears to be wedge-shaped, joint to the disk $\mathbb{F}_{0}$ at one point. As $\nu \downarrow 0$, one obtains a family of $\nu g$ minimal periodic orbits $\left\{\gamma_{\nu}, \dot{\gamma}_{\nu}\right\}$. Forced by the upper semi-continuity, its Kuratowski upper limit set is exactly the Mañé set for the class at the vertex, made up by two minimal homoclinic orbits to the fixed point, looks like a figure eight:

$$
\mathcal{M}(c) \rightarrow \gamma_{i} * \gamma_{i+1}
$$

where $\gamma_{\ell}$ is a minimal homoclinic curve such that $\left[\gamma_{\ell}\right]=g_{\ell}$ for $\ell=i, i+1$.
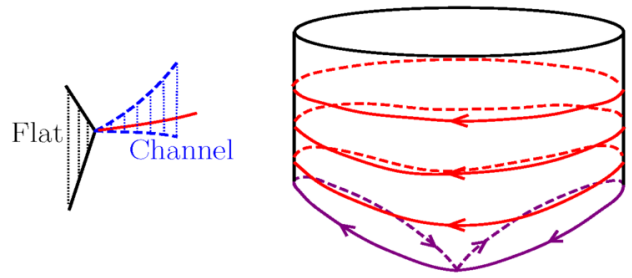

Figure 2: For $c$ in the channel, $\tilde{\mathcal{A}}(c)$ is a closed orbit, it approaches a curve of figure eight, $k_{i}$-folded over $\gamma_{i}$ and $k_{i+1}$-folded over $\gamma_{i+1}$.

To be more precise, let us consider it in the finite covering space $\bar{M}=$ $\bar{k}_{1} \mathbb{T} \times \bar{k}_{2} \mathbb{T}$ where $\bar{k}_{m}=k_{i} g_{i, m}+k_{i+1} g_{i+1, m}$ for $m=1,2$ if we write $g_{\ell}=$ $\left(g_{\ell, 1}, g_{\ell, 2}\right)$ for $\ell=i, i+1$. Let $\sigma:\left\{1,2, \cdots, k_{i}+k_{i+1}\right\} \rightarrow\{i, i+1\}$ be a permutation such that the cardinality $\# \sigma^{-1}(i)=k_{i}$ and $\# \sigma^{-1}(i+1)=$ $k_{i+1}$. The lift of homoclinic curve $\gamma_{i}$ as well as $\gamma_{i+1}$ to $\bar{M}$ contains several curves. Pick up one curve $\bar{\gamma}_{\sigma(1)}$ in the lift of $\gamma_{\sigma(1)}$, it determines a unique curve $\bar{\gamma}_{\sigma(2)}$ such that the end point of $\bar{\gamma}_{\sigma(1)}$ is the starting point of $\bar{\gamma}_{\sigma(2)}$, and so on. We shall see that there exists a unique permutation $\sigma$ (up to a translation) such that, as $c$ approaches the vertex through in channel, each Aubry class in $\mathcal{A}(c, \bar{M})$ approaches (up to a translation) the curve $\bar{\gamma}_{\sigma(1)} * \bar{\gamma}_{\sigma(2)} * \cdots * \bar{\gamma}_{\sigma\left(k_{i}+k_{i+1}\right)}$ without folding. Here $\mathcal{A}(c, \bar{M})$ denotes the Aubry set with respect to $M$. Although $\mathcal{A}(c)$ is made up by one periodic curve, $\mathcal{A}(c, \bar{M})$ may contain several periodic curves.

As the minimal curve $\gamma_{\nu}$ is periodic with the homological class $\left[\gamma_{\nu}\right]=$ $k_{i} g_{i}+k_{i+1} g_{i+1}$, the permutation $\sigma: \mathbb{Z} \rightarrow\{i, i+1\}$ is $\left(k_{i}+k_{i+1}\right)$-periodic. Since $k_{i}$ is prime to $k_{i+1}$, we have $k_{i}=k_{i+1}=1$ if $k_{i}=k_{i+1}$. 
Proposition 2.7. The permutation is uniquely (up to a translation) determined by $k_{i}$ and $k_{i+1}$. For $k_{i}>k_{i+1}, \exists j_{0}$ such that the following holds for $j=1, \cdots, k_{i}+k_{i+1}$

$$
\begin{array}{ll}
\sigma\left(j+j_{0}\right)=i, & \text { if } j=2,4, \cdots, 2 k_{i+1}, 2 k_{i+1}+1, \cdots, k_{i}+k_{i+1}, \\
\sigma\left(j+j_{0}\right)=i+1, & \text { if } j=1,3, \cdots, 2 k_{i+1}-1 .
\end{array}
$$

Proof. By the assumption, there exists only one minimal homoclinic curve $\gamma_{\ell}$ such that $\left[\gamma_{\ell}\right]=g_{\ell}$ for $\ell=i, i+1$. Because of the lemma 2.2, we can assume that $g_{i}=(1,0)$ and $g_{i+1}=(0,1)$ by choosing suitable coordinates on $\mathbb{T}^{2}$. We choose two sections $I^{-}$and $I^{+}$in a small neighborhood of the origin such that, emanating from the origin, these homoclinic curves pass through $I^{-}$ and $I^{+}$successively before they return back to the origin as $t \rightarrow \infty$, see the figure below. In the section $I^{ \pm}$we choose disjoint subsections $I_{i}^{ \pm}$and $I_{i+1}^{ \pm}$ such that the curve $\gamma_{\ell}$ passes through $I_{\ell}^{ \pm}$for $\ell=i, i+1$.
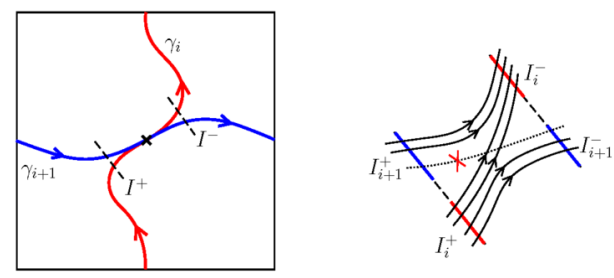

Let $\gamma_{\nu}$ be the minimal periodic curve with rotation vector $\nu g$. For small $\nu>0, \gamma_{\nu}$ falls into a small neighborhood of these two homoclinic curves. So it has to pass either through $I_{i}^{ \pm}$or through $I_{i+1}^{ \pm}$. Let $t_{j}^{ \pm}$be the time for $\gamma_{\nu}$ passing through $I^{ \pm}$with $\cdots<t_{j-1}^{-}<t_{j}^{+}<t_{j}^{-}<t_{j+1}^{+}<\cdots$, and it does not touch these sections whenever $t \neq t_{k}^{ \pm}$. By definition, the period of the curve equals $t_{k_{1}+k_{2}}^{ \pm}-t_{0}^{ \pm}$. If the curve intersects $I_{i}^{+}$at $t_{j}^{+}$and intersects $I_{i+1}^{-}$at $t_{j}^{-}$, then the segment $\left.\gamma_{\nu}\right|_{\left[t_{j-1}^{-}, t_{j}^{+}\right]}$keeps close to $\gamma_{i}$ and $\left.\gamma_{\nu}\right|_{\left[t_{j}^{-}, t_{j+1}^{+}\right]}$keeps close to $\gamma_{i+1}$, so one has $\gamma_{\nu}\left(t_{j-1}^{-}\right) \in I_{i}^{-}$and $\gamma_{\nu}\left(t_{j+1}^{+}\right) \in I_{i+1}^{+}$.

Since the curve $\gamma_{\nu}$ is minimal, it does not have self-intersection. Thus, once there exists $t_{j}^{ \pm}$such that $\gamma_{\nu}\left(t_{j}^{+}\right) \in I_{i}^{+}$and $\gamma_{\nu}\left(t_{j}^{-}\right) \in I_{i}^{-}$, then there does not exist $t_{j^{\prime}}^{ \pm}$such that $\gamma_{\nu}\left(t_{j^{\prime}}^{+}\right) \in I_{i+1}^{+}$and $\gamma_{\nu}\left(t_{j^{\prime}}^{-}\right) \in I_{i+1}^{-}$. Up to a shift, we set $J=\left\{2 k_{i+1}+1,2 k_{i+1}+2, \cdots, k_{i}+k_{i+1}\right\}$ with cardinality $\#(J)=k_{i}-k_{i+1}$ such that for $j \in J$ one has $\gamma_{\nu}\left(t_{j}^{ \pm}\right) \in I_{i}^{ \pm}$, for $j \notin J$ one either has $\gamma_{\nu}\left(t_{j}^{+}\right) \in I_{i}^{+}$ and $\gamma_{\nu}\left(t_{j}^{-}\right) \in I_{i+1}^{-}$or has $\gamma_{\nu}\left(t_{j}^{+}\right) \in I_{i+1}^{+}$and $\gamma_{\nu}\left(t_{j}^{-}\right) \in I_{i}^{-}$. In this way, we obtained a unique permutation $\sigma$ up to a translation.

To make it more clear we introduce a coordinate transformation on $T$ : $\mathbb{T}^{2} \rightarrow \mathbb{T}^{2}$ such that $T_{*} g=g$ for each $g \in H_{1}\left(\mathbb{T}^{2}, \mathbb{Z}\right)$ and $T \gamma_{\nu}$ is a straight 
line projected down to the unit square, a fundamental domain of $\mathbb{T}^{2}$. Starting from a point $z_{0}^{h}=\left(x_{0}, 0\right)$, the line successively reaches to the points $z_{1}^{h}=\left(x_{1}, 0\right), \cdots, z_{m}^{h}=\left(x_{m}, 0\right), \cdots, z_{k_{i}}^{h}=z_{0}^{h}$ where $x_{m}=\left(x_{0}+m k_{i+1} / k_{i}\right.$ mod 1,0) with small $x_{0}>0$. To connect the point $\left(x_{m-1}, 0\right)$ to the point $\left(x_{m}, 1\right)$, the curve $T \gamma_{\nu}$ does not touch the vertical boundary lines if

$$
\left[(m-1) \frac{k_{i+1}}{k_{i}}\right]=\left[m \frac{k_{i+1}}{k_{i}}\right],
$$

where $[a]$ denote the largest integer not bigger than the number $a$, and it has to pass through the vertical lines at some point $z_{m}^{v}=\left(0 \bmod 1, y_{m}\right)$ if

$$
\left[(m-1) \frac{k_{i+1}}{k_{i}}\right]+1=\left[m \frac{k_{i+1}}{k_{i}}\right] .
$$

We define an order $\prec$ for these $k_{i}+k_{i+1}$ points such that $z_{j}^{h} \prec z_{k}^{h}$ iff $j<k$ and $z_{j}^{h} \prec z_{j+1}^{v} \prec z_{j+1}^{h}$ iff $\left[j k_{i} / k_{j+1}\right]+1=\left[(j+1) k_{i} / k_{j+1}\right]$.
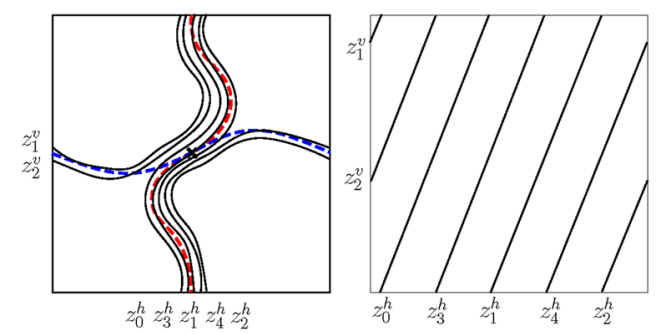

Back to the original coordinates, the curve $\gamma_{\nu}$ falls into a neighborhood of the curves $\gamma_{i}$ and $\gamma_{i+1}$, intersects the horizontal line $\Gamma_{h}=T^{-1}\left\{\left(x_{1}, x_{2}\right)\right.$ : $\left.x_{1}=\frac{1}{2} \bmod 1\right\}$ at $T^{-1} z_{j}^{h}$ and intersects the vertical line $\Gamma_{v}=T^{-1}\left\{\left(x_{1}, x_{2}\right)\right.$ : $\left.x_{2}=\frac{1}{2} \bmod 1\right\}$ at $T^{-1} z_{j}^{v},\left[\Gamma_{h}\right]=g_{i+1}$ and $\left[\Gamma_{v}\right]=g_{i}$. The map $T$ naturally induces the order among these points: $T^{-1} z_{j}^{h, v} \prec T^{-1} z_{k}^{h, v}$ if and only if $z_{j}^{h, v} \prec z_{k}^{h, v}$. If the curve passes the point $T^{-1} z_{j}^{h}$ at $t \in\left(t_{j}^{-}, t_{j+1}^{+}\right)$, the segment $\left.\gamma_{\nu}\right|_{\left[t_{j}^{-}, t_{j+1}^{+}\right]}$falls into a neighborhood of $\gamma_{i}$, otherwise, it falls into a neighborhood of $\gamma_{i+1}$.

\section{Cohomology equivalence around the flat $\mathbb{F}_{0}$}

As it was done in [CY1, CY2, LC], the orbits connecting two Aubry sets are constructed by variational method shadowing a sequence of local connecting orbits. Two types of local connecting orbits are found for this purpose, one is the variational version of Arnold's mechanism and the other is based on the principle of cohomology equivalence. 


\subsection{A new version of cohomology equivalence}

In [M93], Mather introduced the concept of cohomology equivalence for the construction of connecting orbit. However, it does not apply to interesting problem in autonomous systems and one tries to modify the notion (see for example [B02, B08]).

A new version of cohomology equivalence was introduced in $[\mathrm{LC}]$ for autonomous system. For a Tonelli Lagrangian defined on $T \mathbb{T}^{n}$, it is defined not with respect to the whole $\mathbb{T}^{n}$ as in [M93], but to a section. For $n$-torus $\mathbb{T}^{n}$, the section is chosen as a non-degenerately embedded section $(n-1)$-dimensional torus. We call $\Sigma_{c}$ non-degenerately embedded $(n-1)$-dimensional torus by assuming a smooth injection $\varphi: \mathbb{T}^{n-1} \rightarrow \mathbb{T}^{n}$ such that $\Sigma_{c}$ is the image of $\varphi$, and the induced map $\varphi_{*}: H_{1}\left(\mathbb{T}^{n-1}, \mathbb{Z}\right) \rightarrow H_{1}\left(\mathbb{T}^{n}, \mathbb{Z}\right)$ is an injection.

Let $\mathfrak{C} \subset H^{1}\left(\mathbb{T}^{n}, \mathbb{R}\right)$ be a set where we are going to define cohomology equivalence. For each class $c \in \mathfrak{C}$, we assume that there exists a nondegenerate embedded $(n-1)$-dimensional torus $\Sigma_{c} \subset \mathbb{T}^{n}$ such that each $c$-semi static curve $\gamma$ transversally intersects $\Sigma_{c}$. Let

$$
\mathbb{V}_{c}=\bigcap_{U}\left\{i_{U *} H_{1}(U, \mathbb{R}): U \text { is a neighborhood of } \mathcal{N}(c) \cap \Sigma_{c}\right\},
$$

here $i_{U}: U \rightarrow M$ denotes inclusion map. $\mathbb{V}_{c}^{\perp}$ is defined to be the annihilator of $\mathbb{V}_{c}$, i.e. if $c^{\prime} \in H^{1}\left(\mathbb{T}^{n}, \mathbb{R}\right)$, then $c^{\prime} \in \mathbb{V}_{c}^{\perp}$ if and only if $\left\langle c^{\prime}, h\right\rangle=0$ for all $h \in \mathbb{V}_{c}$. Clearly,

$$
\mathbb{V}_{c}^{\perp}=\bigcup_{U}\left\{\operatorname{ker} i_{U}^{*}: U \text { is a neighborhood of } \mathcal{N}(c) \cap \Sigma_{c}\right\} .
$$

Note that there exists a neighborhood $U$ of $\mathcal{N}(c) \cap \Sigma_{c}$ such that $\mathbb{V}_{c}=$ $i_{U *} H_{1}(U, \mathbb{R})$ and $\mathbb{V}_{c}^{\perp}=\operatorname{ker}_{U}^{*}$ (see [M93]).

We say that $c, c^{\prime} \in H^{1}\left(\mathbb{T}^{n}, \mathbb{R}\right)$ are cohomologically equivalent if there is a continuous curve $\Gamma:[0,1] \rightarrow \mathfrak{C}$ such that $\Gamma(0)=c, \Gamma(1)=c^{\prime}, \alpha(\Gamma(s))$ keeps constant along $\Gamma$, and for each $s_{0} \in[0,1]$ there exists $\delta>0$ such that $\Gamma(s)-\Gamma\left(s_{0}\right) \in \mathbb{V} \frac{\perp}{\Gamma\left(s_{0}\right)}$ whenever $s \in[0,1]$ and $\left|s-s_{0}\right|<\delta$.

It is proved in $[\mathrm{LC}]$ that the Aubry set $\tilde{\mathcal{A}}(c)$ is dynamically connected to the Aubry set $\tilde{\mathcal{A}}\left(c^{\prime}\right)$ if the class $c$ is equivalent to $c^{\prime}$.

Here, we extend the cohomology equivalence for autonomous system to time-periodic system and obtain a new version if we treat the time $t$ as a new angle variable and choose a section in the extended configuration space $\mathbb{T}^{n+1}$ where the extra dimension is for $t$. If we write the cohomology class in 
coordinates $\left(c_{1}, \cdots, c_{n}, c^{*}\right), c_{i}$ corresponds to $x_{i}$, then $c^{*}=\alpha(c)$ corresponds to $t$. It appears to be more flexible than that introduced by Mather, which turns out to be a special case of the new version where the section is chosen as the time-0-section.

To illustrate how two Aubry sets are dynamically connected by the mechanism of cohomology equivalence, we consider the Lagrangian system (1.1).

Lemma 3.1. Given $c \in H^{1}\left(\mathbb{T}^{2}, \mathbb{R}\right)$ such that $\alpha_{0}(c)=E>\min \alpha_{0}$ we assume that the Mañé set $\tilde{\mathcal{N}}(c)$ for $L_{0}$ does not project surjectively on $\mathbb{T}^{2}$. Then there exist $\delta>0$ and $\epsilon_{0}>0$ such that for each $\epsilon \in\left[0, \epsilon_{0}\right]$ and $c^{\prime} \in \alpha_{\epsilon}^{-1}\left(\alpha_{\epsilon}(c)\right)$ with $\left|c^{\prime}-c\right|<\delta$, the Aubry sets $\tilde{\mathcal{A}}(c)$ and $\tilde{\mathcal{A}}\left(c^{\prime}\right)$ are dynamically connected.

Proof. Let $\omega(\mu)=\left(\omega_{1}(\mu), \omega_{2}(\mu)\right)$ denote the rotation vector of the measure $\mu$, which obviously depends on the coordinate system. As $\alpha_{0}(c)>\min \alpha_{0}$, there is a line passing through the origin $\omega=0 \in H_{1}\left(\mathbb{T}^{2}, \mathbb{R}\right)$ such that the convex set $\mathscr{L}_{\beta_{0}}^{-1}(c)$ stays one side of the line. So, there is a coordinate transformation $x \rightarrow M x$ where $M$ is a uni-module matrix such that in the new coordinates $\omega_{1}\left(\mu_{c}\right)>0$ holds for each ergodic $c$-minimal measure $\mu_{c}$.

By the condition, there exists a section $S_{c}$ of $\mathbb{T}^{2}$ homotopic to $\left\{\left(x_{1}, x_{2}\right)\right.$ : $\left.x_{1}=0\right\}$ such that $S_{c} \cap \mathcal{N}_{L_{0}}(c) \subset \operatorname{int} \cup I_{c, i}$ where we use the notation $\mathcal{N}_{L_{2}}(c)$ to denote the Mañé set for $L_{\imath}$ at $c$ with $\imath=0, \epsilon,\left\{I_{c, i}\right\}$ denote disjoint closed intervals. In the extended configuration space $\mathbb{T}^{3}$ we choose $\Sigma_{c}=S_{c} \times \mathbb{T}$.

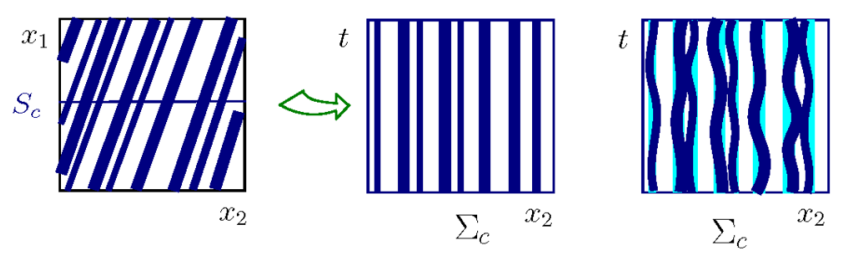

Figure 3 .

Because of the upper semi-continuity of the Mañé set in the Lagrangian and in the cohomology class, there exist $\delta, \epsilon_{0}>0$ such that for each $\epsilon \in\left[0, \epsilon_{0}\right]$ and $\left|c^{\prime}-c\right| \leq \delta$ the following

$$
\mathcal{N}_{L_{\epsilon}}\left(c^{\prime}\right) \cap \Sigma_{c} \subset\left(\cup I_{c, i}\right) \times \mathbb{T}
$$

holds in the extended configuration space. Treating $\left(c, \alpha_{\epsilon}(c)\right)$ as a class for the extended space, one has $V_{c, \alpha_{\epsilon}(c)}=\operatorname{span}\{(0,0,1)\}$, from which one obtains

$$
\left(c^{\prime}, \alpha_{\epsilon}\left(c^{\prime}\right)\right)-\left(c, \alpha_{\epsilon}(c)\right) \in V_{c, \alpha_{\epsilon}(c)}^{\perp}, \quad \text { if } \alpha_{\epsilon}\left(c^{\prime}\right)=\alpha_{\epsilon}(c) .
$$


Regarding each $I_{c, i}$ as a segment of line embedded in the torus $\mathbb{T}^{2}$, we find that the set $\cup I_{c, i}$ is topologically trivial. Therefore, there exists a smooth function $u: \mathbb{T}^{2} \rightarrow \mathbb{R}$ such that $u=\left\langle x, c^{\prime}-c\right\rangle$ when it is restricted in a neighborhood $U_{c}=[-d, d] \times \cup I_{c, i}$. The 1-form $\mu=\left\langle c^{\prime}-c, d x\right\rangle-d u$ is closed with $[\mu]=c^{\prime}-c$ and its support does not touch $U_{c}$. We can choose $u$ so that $|u|_{C^{2}}$ is sufficiently small provided $\left|c^{\prime}-c\right|$ is sufficiently small.

To construct orbits connecting $\tilde{\mathcal{A}}(c)$ to $\tilde{\mathcal{A}}\left(c^{\prime}\right)$, we work in the covering space $\bar{M}=\mathbb{R} \times \mathbb{T} \ni\left(\bar{x}_{1}, \bar{x}_{2}\right)=\bar{x}$ in the new coordinates, where $\bar{x}_{1} \in \mathbb{R}$ and $\bar{x}_{2} \in \mathbb{T}$.

We can think the Lagrangian $L_{\epsilon}$ and the 1-form $\eta_{c}$ to be defined on the covering space, just think it is periodic in $x_{1}$. Let $\rho: \bar{M} \rightarrow \mathbb{R}$ be a smooth function such that $\rho=1$ for $\bar{x}_{1} \geq d$ and $\rho=0$ for $\bar{x}_{1} \leq-d$. The lift of the section $\Sigma_{c}$ to the covering space contains infinitely many connected components, denoted by $\left\{\Sigma_{i}: \bar{x}_{1}=i\right\}$. Let $\bar{M}^{+}=\left\{\bar{x}: \bar{x}_{1} \geq 1\right\}$ and $\bar{M}^{-}=\left\{\bar{x}: \bar{x}_{1} \leq-1\right\}$. For $\bar{x} \in \bar{M}^{-}$and $\bar{x}^{\prime} \in \bar{M}^{+}$, we set

$$
h_{L_{\epsilon}, \eta_{c}, \rho \mu}\left(\bar{x}, \bar{x}^{\prime}\right)=\inf _{k \in \mathbb{Z}_{+}} \inf _{\substack{\bar{\gamma}(-k)=\bar{x} \\ \bar{\gamma}(k)=\bar{x}^{\prime}}}\left[A_{L_{\epsilon}, \eta_{c}, \rho \mu}(\bar{\gamma})\right],
$$

where

$$
\left[A_{L_{\epsilon}, \eta_{c}, \rho \mu}(\bar{\gamma})\right]=\int_{-k}^{k}\left(L_{\epsilon}-\eta_{c}-\rho \mu\right)(\bar{\gamma}(s), \dot{\bar{\gamma}}(s), s) d s+2 k \alpha_{\epsilon}(c) .
$$

If $c^{\prime}$ is close to $c$ such that $\omega_{1}\left(\mu^{\prime}\right)>0$ holds for each ergodic $c^{\prime}$-minimal measure $\mu^{\prime}$, the infimum is reached at some $k<+\infty$. To see why, let $\bar{\gamma}_{k}:[-k, k] \rightarrow \bar{M}$ be the minimal curve of $\inf _{\bar{\gamma}(-k)=\bar{x}, \bar{\gamma}(k)=\bar{x}^{\prime}}\left[A_{L_{\epsilon}, \eta_{c}, \rho \mu}(\gamma)\right]$. If $h_{L_{\epsilon}, \eta_{c}, \rho \mu}\left(\bar{x}, \bar{x}^{\prime}\right)$ is attained as $k \rightarrow \infty$, the orbit $\left(\bar{\gamma}_{k}, \overline{\bar{\gamma}}_{k}\right)$ will accumulate some invariant measure $\mu$ such that $\omega_{1}(\mu)=0$ if we write $\omega(\mu)=\left(\omega(\mu)_{1}, \omega(\mu)_{2}\right)$. For both $c^{*}=c, c^{\prime}$, the average action of $L_{\epsilon}-\left\langle c^{*}, \dot{x}\right\rangle+\alpha_{\epsilon}\left(c^{*}\right)$ over this measure is bigger than the action over the minimal measure with $\omega_{1}>0$ which is equal to zero. Consequently, one would have $h_{L_{\epsilon}, \eta_{c}, \rho \mu}\left(\bar{x}, \bar{x}^{\prime}\right)=\infty$. For the details, one can refer to the proof of Lemma 2.1 in [LC].

With positive integers $\ell$ and $\ell^{\prime}$ we define the Deck transformations $\ell^{*}$ : $\bar{M} \rightarrow \bar{M}: \ell^{*} \bar{x}=\left(\bar{x}_{1}-\ell, \bar{x}_{2}\right)$ and $\ell^{\prime *} \bar{x}^{\prime}=\left(\bar{x}_{1}^{\prime}+\ell^{\prime}, \bar{x}_{2}^{\prime}\right)$. Let $\bar{\gamma}_{\ell, \ell^{\prime}}$ be the minimal curve for $h_{\epsilon, c^{\prime}, c}\left(\ell^{*} \bar{x}, \ell^{\prime *} \bar{x}^{\prime}\right)$ which is defined on the interval $\left[-k\left(\ell, \ell^{\prime}\right), k\left(\ell, \ell^{\prime}\right)\right]$. Because of super-linear growth of the Lagrangian, one has $k\left(\ell, \ell^{\prime}\right) \rightarrow \infty$ as $\ell, \ell^{\prime} \rightarrow \infty$.

Let $\bar{\gamma}_{\ell_{i}, \ell_{j}^{\prime}}:\left[-k\left(\ell_{i}, \ell_{j}^{\prime}\right), k\left(\ell_{i}, \ell_{j}^{\prime}\right)\right] \rightarrow \bar{M}$ be the sequence of minimal curves such that

$$
\liminf _{\ell, \ell^{\prime} \rightarrow \infty} h_{\epsilon, c^{\prime}, c}\left(\ell^{*} \bar{x}, \ell^{\prime *} \bar{x}^{\prime}\right)=\lim _{\ell_{i}, \ell_{j}^{\prime} \rightarrow \infty}\left[\left.A_{L_{\epsilon}, \eta_{c}, \rho \mu}\left(\bar{\gamma}_{\ell_{i}, \ell_{j}^{\prime}}\right)\right|_{\left[-k\left(\ell, \ell^{\prime}\right), k\left(\ell, \ell^{\prime}\right)\right]}\right] .
$$


For any large $T$, there exists $i_{0}>0$ such that $k\left(\ell_{i}, \ell_{j}^{\prime}\right)>T$ holds for all $\ell_{i}, \ell_{j}^{\prime} \geq i_{0}$ and the set $\left\{\left.\bar{\gamma}_{\ell_{i}, \ell_{j}^{\prime}}\right|_{[-T, T]}: \ell_{i}, \ell_{j}^{\prime} \geq i_{0}\right\}$ is pre-compact in $C^{1}([-T, T], \bar{M})$. Let $T \rightarrow \infty$, by the diagonal extraction argument, there is a subsequence of $\left\{\bar{\gamma}_{\ell_{i}, \ell_{j}^{\prime}}\right\}$ which $C^{1}$-converges on each compact set to a $C^{1}$-curve $\bar{\gamma}: \mathbb{R} \rightarrow \bar{M}$. Let $\mathscr{C}_{L_{\epsilon}, \eta_{c}, \rho \mu}$ denote the set of all curves obtained in this way. Let

$$
\tilde{\mathcal{C}}_{L_{\epsilon}, \eta_{c}, \rho \mu}=\bigcup_{\bar{\gamma} \in \mathscr{C}_{L_{\epsilon}, \eta_{c}, \rho \mu}}(\bar{\gamma}(t), \dot{\bar{\gamma}}(t), t), \quad \mathcal{C}_{L_{\epsilon}, \eta_{c}, \rho \mu}=\bigcup_{\bar{\gamma} \in \mathscr{C}_{L_{\epsilon}, \eta_{c}, \rho \mu}}(\bar{\gamma}(t), t)
$$

let $\pi_{1}: \mathbb{R} \times \mathbb{T} \rightarrow \mathbb{T}^{2}$ be the standard projection, then one has

1. let $\gamma=\pi_{1} \bar{\gamma}$, for each $\bar{\gamma} \in \mathscr{C}_{L_{\epsilon}, \eta_{c}, \rho \mu}$, the orbit $(\gamma, \dot{\gamma})$ has $\tilde{\mathcal{A}}(c)$ and $\tilde{\mathcal{A}}\left(c^{\prime}\right)$ as its $\alpha$-limit, $\omega$-limit set respectively;

2. if $\mu=0$, then $\pi_{1} \tilde{\mathcal{C}}_{L_{\epsilon}, \eta_{c}, \rho \mu}=\tilde{\mathcal{N}}(c)$ and $\pi_{1} \mathcal{C}_{L_{\epsilon}, \eta_{c}, \rho \mu}=\mathcal{N}(c)$;

3. both $\tilde{\mathcal{C}}_{L_{\epsilon}, \eta_{c}, \rho \mu}$ and $\mathcal{C}_{L_{\epsilon}, \eta_{c}, \rho \mu}$ are upper-semi continuous in $\left(L_{\epsilon}, \eta_{c}, \rho \mu\right)$.

The first two points are obvious. The third one follows from the observation: restricted on any large interval $[-T, T]$ the set $\left\{\bar{\gamma}_{i}\right\}$ is $C^{1}$-precompact, where $\bar{\gamma}_{i} \in \mathscr{C}_{L_{\epsilon, i}, \eta_{c}, \rho \mu_{i}}$ and $\left(L_{\epsilon, i}, \eta_{c}, \rho \mu_{i}\right) \rightarrow\left(L_{\epsilon}, \eta_{c}, \rho \mu\right)$. The accumulation point must be in $\mathscr{C}_{L_{\epsilon}, \eta_{c}, \rho \mu}$.

Because of the choice of $d u$, each curve $\bar{\gamma}$ of $\mathscr{C}_{L_{0}, \eta_{c}, 0}$ does not touch the support of $d u$ when it passes through the set $\Sigma_{0}+d=\left\{\left|\bar{x}_{1}\right| \leq d\right\}$. Due to the upper semi-continuity, such property remains true if $\epsilon$ and $\left|c^{\prime}-c\right|$ is suitably small, see the figure on the right in Fig. 3. One can construct small $\rho d u$ provided $\left|c^{\prime}-c\right|$ is small. Therefore, along each curve $\bar{\gamma} \in \mathscr{C}_{L_{\epsilon}, \eta_{c}, \rho \mu}$ the term $\rho d u$ does not contribute to the Lagrange equation of $L_{\epsilon}-\eta_{c}-\rho \mu$, namely, $(\gamma, \dot{\gamma})$ is an orbit of $\phi_{L_{\epsilon}}^{t}$, the Euler-Lagrange flow determined by $L_{\epsilon}$, connecting $\tilde{\mathcal{A}}(c)$ to $\tilde{\mathcal{A}}\left(c^{\prime}\right)$.

\subsection{Topology of orbits in the lowest energy level set}

Before getting involved into the details about how such cohomology equivalence is found, let us establish a lemma, reminiscent of Shilnikov's $\lambda$-lemma. It is technically crucial for the follow-up demonstration. For $\theta>0$ and $v \in \mathbb{R}^{n} \backslash\{0\}$, we define a cone

$$
C(v, \theta, d)=\left\{x \in \mathbb{R}^{n}:|\langle x, v\rangle| \geq \theta\|v\|\|x\|,\|x\|=d\right\} .
$$

Lemma 3.2. For mechanical Hamiltonian of $G=\frac{1}{2}\left\langle A^{-1} y, y\right\rangle-V(x)$, we assume that $(x, y)=(0,0) \in\left\{G^{-1}(0)\right\}$ is a hyperbolic fixed point for $\Phi_{G}^{t}$, 
where all eigenvalues are real and different:

$$
\operatorname{Spec}\{J \nabla G(0,0)\}=\left\{ \pm \lambda_{1}, \pm \lambda_{2} ; 0<\lambda_{1}<\lambda_{2}\right\} .
$$

Let $\left(\Lambda_{i, x}^{ \pm}, \Lambda_{i, y}^{ \pm}\right)$be the eigenvector for $\pm \lambda_{i}$, where $\Lambda_{i, x}^{ \pm}, \Lambda_{i, y}^{ \pm}$denote the $x$-, $y$-coordinate component respectively. Let $(x(t), y(t)) \subset\left\{G^{-1}(0)\right\}$ be an orbit such that $x(t)$ passes through a ball $B_{\delta}(0) \subset \mathbb{R}^{n}$ with $x(-T) \in \partial B_{\delta}(0)$, $x(T) \in \partial B_{\delta}(0)$ and $x(t) \in \operatorname{int} B_{\delta}(0)$ for all $t \in(-T, T)$. Then, for small $\delta>0$ and $\theta \geq \frac{1}{2}$, there exist sufficiently large $T_{\theta}>0$ such that for $T \geq T_{\theta}$ one has

$$
(x(-T), x(T)) \notin C\left(\Lambda_{1, x}^{+}, \theta, \delta\right) \times C\left(\Lambda_{1, x}^{-}, \theta, \delta\right) .
$$

If $x( \pm T) \in C\left(\Lambda_{1, x}^{ \pm}, \theta, \delta\right)$, for $T \rightarrow \infty$, there exist constant $d_{2}^{+}, d_{2}^{-}$such that

$$
\left|x(\mp T)-d_{2}^{\mp} \Lambda_{2, x}^{\mp}\right|=o(\delta) .
$$

Proof. By some symplectic transformation of coordinates, the Hamiltonian is reduced to the normal form

$$
G^{\prime}(x, y)=\sum_{i=1}^{2} \frac{1}{2}\left(y_{i}^{2}-\lambda_{i}^{2} x_{i}^{2}\right)+P_{3}(x, y)
$$

where $P_{3}(x, y)=O\left(|(x, y)|^{3}\right)$. By the method of variation of constants, we obtain the solution of the Hamilton equation produced by the normal form

$$
\begin{aligned}
& x_{i}(t)=e^{-\lambda_{i} t}\left(b_{i}^{-}+F_{i}^{-}\right)+e^{\lambda_{i} t}\left(b_{i}^{+}+F_{i}^{+}\right), \\
& y_{i}(t)=-\lambda_{i} e^{-\lambda_{i} t}\left(b_{i}^{-}+F_{i}^{-}\right)+\lambda_{i} e^{\lambda_{i} t}\left(b_{i}^{+}+F_{i}^{+}\right),
\end{aligned}
$$

where $b_{i}^{ \pm}$are constants determined by boundary condition and

$$
\begin{aligned}
F_{i}^{-} & =\frac{1}{2 \lambda_{i}} \int_{0}^{t} e^{\lambda_{i} s}\left(\lambda_{i} \partial_{y_{i}} P_{3}+\partial_{x_{i}} P_{3}\right)(x(s), y(s)) d s \\
F_{i}^{+} & =\frac{1}{2 \lambda_{i}} \int_{0}^{t} e^{-\lambda_{i} s}\left(\lambda_{i} \partial_{y_{i}} P_{3}-\partial_{x_{i}} P_{3}\right)(x(s), y(s)) d s
\end{aligned}
$$

Be aware that autonomous Hamiltonian keeps constant along its orbits. Substituting the formula (3.3) at $t=0$ for the variable $(x, y)$ in the normal form, we get a constraint for the constants $b_{i}^{ \pm}$:

$$
G^{\prime}(x(t), y(t))=-2 \sum_{i=1}^{2} \lambda_{i}^{2} b_{i}^{-} b_{i}^{+}+P_{3}\left(\left(b_{i}^{+}+b_{i}^{-}\right), \lambda_{i}\left(b_{i}^{+}-b_{i}^{-}\right)\right) .
$$


Let $z=(x, y)$ and let $e^{\mathrm{A} t} z$ denote the flow of the linearized equation $\dot{z}=$ $J \nabla G^{\prime}(0) z$, where $\mathrm{A}=J \nabla G^{\prime}(0)$. Although a conjugacy $h=\mathrm{id}+v$ between the Hamiltonian flow $\Phi_{G^{\prime}}^{t}(z)$ and the linear flow $e^{\mathrm{A} t} z$ is only $C^{0}$, according to Hartman-Grobman Theorem, we can see from the proof that $|v(z)|=$ $o(|z|)$ when $|z|$ is small (we will do it later). From the conjugate relation $\Phi_{G^{\prime}}^{t}(h(z))=e^{\mathrm{A} t} z+v\left(e^{\mathrm{A} t} z\right)$ we find the solution of the Hamiltonian equation takes the form

$$
\begin{aligned}
& x_{i}(t)=b_{i}^{-} e^{-\lambda_{i} t}+b_{i}^{+} e^{\lambda_{i} t}+o(\delta), \\
& y_{i}(t)=\lambda_{i}\left(-b_{i}^{-} e^{-\lambda_{i} t}+b_{i}^{+} e^{\lambda_{i} t}\right)+o(\delta),
\end{aligned}
$$

provided $|x(t)|,|y(t)| \leq \delta$. Given a boundary condition $x(-T), x(T)$ with $|x( \pm T)|=\delta$ with small $\delta>0$, there is a unique solution $x(t), y(t)$ such that $|x(t)|,|y(t)| \leq \delta$ for $t \in[-T, T]$ provided $T>0$ is suitably large.

Let us investigate how the constants $b_{i}^{ \pm}$depend on the boundary condition $x(T)=\left(x_{1}^{+}, x_{2}^{+}\right) \in \partial B_{\delta}(0), x(-T)=\left(x_{1}^{-}, x_{2}^{-}\right) \in \partial B_{\delta}(0)$ by assuming

$$
\min \left\{\left|x_{1}^{-}\right|,\left|x_{1}^{+}\right|\right\} \geq \frac{\delta}{2} .
$$

For $\theta=1 / 2,(x(-T), x(T)) \in C\left(\Lambda_{1, x}^{+}, \theta, \delta\right) \times C\left(\Lambda_{1, x}^{-}, \theta, \delta\right)$ implies (3.6) holds. Because the curve $\left.x\right|_{[-T, T]}$ stays inside of the ball $B_{\delta}(0)$ and $T$ is sufficiently large, the orbit $\left.(x, y)\right|_{[-T, T]}$ stays near the stable and unstable manifold of the fixed point. From (3.5) one immediately obtains the solution

$$
\begin{aligned}
& b_{i}^{-}=\frac{e^{-\lambda_{i} T}\left(x_{i}^{-}+o(\delta)\right)-e^{-3 \lambda_{i} T}\left(x_{i}^{+}+o(\delta)\right)}{1-e^{-4 \lambda_{i} T}}, \\
& b_{i}^{+}=\frac{e^{-\lambda_{i} T}\left(x_{i}^{+}+o(\delta)\right)-e^{-3 \lambda_{i} T}\left(x_{i}^{-}+o(\delta)\right)}{1-e^{-4 \lambda_{i} T}} .
\end{aligned}
$$

For sufficiently large $T>0$, it follows from the assumption (3.6) and Equation (3.7) that

$$
\left|b_{1}^{ \pm}\right| \geq \frac{\delta}{3} e^{-\lambda_{1} T}, \quad\left|b_{2}^{ \pm}\right| \leq 2 \delta e^{-\lambda_{2} T} .
$$

It follows from $\lambda_{1}<\lambda_{2}$ that $\left|b_{2}^{ \pm}\right| \ll\left|b_{1}^{ \pm}\right|$if $T$ is sufficiently large. In this case, we get from (3.4) that

$$
|G(x(t), y(t))|>\left|\lambda_{1}^{2} b_{1}^{+} b_{1}^{-}\right|>0 .
$$

It contradicts the assumption that $(x(t), y(t)) \in\left\{G^{-1}(0)\right\}$. Let $T \rightarrow \infty$, one obtains the last item immediately. 
To complete the proof, let us verify Formula (3.5). Consider the time1-map defined by $e^{\mathrm{A} t} z$ and $\Phi_{G^{\prime}}^{t}(z)$ and let $B z=e^{\mathrm{A}} z,\left.\Phi_{G^{\prime}}^{t}\right|_{t=1}=B z+g(z)$. Since

$$
\frac{d}{d t}\left(\Phi_{G^{\prime}}^{t}(z)-e^{\mathrm{A} t} z\right)=\mathrm{A}\left(\Phi_{G^{\prime}}^{t}(z)-e^{\mathrm{A} t} z\right)+J \nabla P_{3}\left(\Phi_{G^{\prime}}^{t}(z)\right)
$$

by using the method of variation of constants one obtains a formal solution

$$
\Phi_{G^{\prime}}^{t}(z)-e^{\mathrm{A} t} z=e^{\mathrm{A} t} \int_{0}^{t} e^{-\mathrm{A} s} J \nabla P_{3}\left(\Phi_{G^{\prime}}^{s}(z)\right) d s .
$$

one has $\left|\Phi_{G^{\prime}}^{t}(z)-e^{\mathrm{A} t} z\right| \leq \sup \left|J \nabla P_{3}\left(\Phi_{G^{\prime}}^{t}(z)\right)\right|\left|\mathrm{A}^{-1}\left(e^{\mathrm{A} t}-1\right)\right|$. Since $\left|\nabla P_{3}(z)\right|=$ $O\left(|z|^{2}\right)$, one obtains $|g(z)|=O\left(|z|^{2}\right)$.

In the proof of Hartman-Grobman Theorem, the conjugacy id $+v$ solves the equation

$$
(B+g) \circ(\mathrm{id}+v)=(\mathrm{id}+v) \circ B,
$$

which is equivalent to the equation

$$
\Psi(g, v)=v-B \circ v \circ B^{-1}-g \circ(\mathrm{id}+v) \circ B^{-1}=0
$$

Since $B$ is hyperbolic, the linear map $v \rightarrow v-B \circ v \circ B^{-1}: C^{0}\left(\mathbb{R}^{n}, \mathbb{R}^{n}\right) \rightarrow$ $C^{0}\left(\mathbb{R}^{n}, \mathbb{R}^{n}\right)$ is an isomorphism. Due to the Implicit Function Theorem, for small $g$ the equation $\Psi(g, v)=0$ has a unique solution $v$. Since $v$ is continuous and $v(0)=0, v(z) \rightarrow 0$ as $z \rightarrow 0$. It follows from $g(z)=O\left(|z|^{2}\right)$ that $|v(z)|=o(|z|)$ as $|z|$ is small.

Because of the relation between the eigenvalues $\left|\lambda_{1}\right|<\left|\lambda_{2}\right|$, on the unstable manifold there exist exactly two orbits $\left(\gamma^{-}(t), \dot{\gamma}^{-}(t)\right)$ and $\left(\gamma^{\prime-}(t), \dot{\gamma}^{\prime-}(t)\right)$ which approach the origin as $t \rightarrow-\infty$ in the direction of $\Lambda_{2, x}$ :

$$
\lim _{t \rightarrow-\infty} \frac{\dot{\gamma}^{-}(t)}{\left|\dot{\gamma}^{-}(t)\right|}=\Lambda_{2, x}, \quad \lim _{t \rightarrow-\infty} \frac{\dot{\gamma}^{\prime-}(t)}{\left|\dot{\gamma}^{\prime-}(t)\right|}=-\Lambda_{2, x} .
$$

On the stable manifold there exist exactly two orbits $\left(\gamma^{+}(t), \dot{\gamma}^{+}(t)\right)$ and $\left(\gamma^{\prime+}(t), \dot{\gamma}^{\prime+}(t)\right)$ which approach the origin as $t \rightarrow \infty$ in the direction of $\Lambda_{2, x}$ :

$$
\lim _{t \rightarrow \infty} \frac{\dot{\gamma}^{+}(t)}{\left|\dot{\gamma}^{+}(t)\right|}=\Lambda_{2, x}, \quad \lim _{t \rightarrow \infty} \frac{\dot{\gamma}^{\prime+}(t)}{\left|\dot{\gamma}^{\prime+}(t)\right|}=-\Lambda_{2, x} .
$$

These curves intersect the circle $\partial B_{\delta}(0)$ at four points: $x_{\delta}^{ \pm}$is the intersection point of $\gamma^{ \pm}$with $\partial B_{\delta}(0)$ and $x_{\delta}^{\prime \pm}$ is the intersection point of $\gamma^{\prime \pm}$ with $\partial B_{\delta}(0)$. Obviously, for small $\delta>0, x_{\delta}^{+}$is close to $x_{\delta}^{-}$and $x_{\delta}^{\prime+}$ is close to $x_{\delta}^{\prime-}$. 


\subsection{The Mañé set for $c \in \partial \mathbb{F}_{0}$}

To establish the cohomology equivalence around double resonant point, let us first consider the classical Lagrangian with two degrees of freedom

$$
L_{0}(x, \dot{x})=\frac{1}{2}\langle A \dot{x}, \dot{x}\rangle+V(x) .
$$

Recall the flat $\mathbb{F}_{0}=\alpha_{0}^{-1}\left(\min \alpha_{0}\right)$ where $\alpha_{0}$ denotes the $\alpha$-function of $L_{0}$. The main goal of this subsection is to prove

Theorem 3.1. For the Lagrangian $L_{0}$, there exists a residual set $\mathfrak{V} \subset$ $C^{r}\left(\mathbb{T}^{2}, \mathbb{R}\right)$ with $r \geq 2$ such that for each $V \in \mathfrak{V}$ it holds simultaneously for all $c \in \partial \mathbb{F}_{0}$ that the Mañé set does not project surjectively on the configuration space: $\mathcal{N}(c) \subsetneq \mathbb{T}^{2}$.

Proof. We shall show in the following that, if $\mathcal{N}(c)=\mathbb{T}^{2}$, there is some $c$ semi static curve approaching the origin $\{x=0\}$ in the direction of $\pm \Lambda_{2, x}$, namely, one of the formulae in (3.8) and (3.9) holds for this curve.

There are only four orbits $\left(\gamma^{ \pm}(t), \dot{\gamma}^{ \pm}(t)\right),\left(\gamma^{\prime \pm}(t), \dot{\gamma}^{\prime \pm}(t)\right)$ approaching the fixed point in the direction of $\pm \Lambda_{2}$ as $t \rightarrow \infty$ or as $t \rightarrow-\infty$. These orbits connect the fixed point to at most four Aubry classes. Because of the work $[\mathrm{BC}]$ it is a $C^{\infty}$-generic property that, for each first cohomology class, the Mather set contains at most three connected components. Since each Aubry class is compact, some $\delta>0$ exists so that $\bar{B}_{\delta}(0)$ is disjoint with these Aubry sets, unless one of them is the fixed point itself.

In the disk $B_{\delta}(0)$, there exists a smaller disk $U$ such that none of the curves $\gamma^{ \pm}, \gamma^{\prime \pm}$ touch $\bar{U}$ provided it is semi-static, because the duration of a semi static curve staying outside of $\delta$-neighborhood of Mather set is finite.

We construct potential perturbation $V \rightarrow V+V_{\delta}$ such that non-negative $V_{\delta}$ is small in $C^{r}$-topology and $\operatorname{supp} V_{\delta} \subseteq \bar{U}$. In this way, these Aubry classes remains unchanged. Since $V_{\delta}$ is non-negative, each curve of $\gamma^{ \pm}, \gamma^{\prime \pm}$ remains semi-static for certain $c$ if it is already semi-static before the perturbation is added. So, if $\mathcal{N}(c)=\mathbb{T}^{2}$, under the small perturbation, none of the $c$-semistatic curves passes through the set $U$.

It is possible that, if the perturbation is added, another curve of $\gamma^{ \pm}, \gamma^{\prime \pm}$ becomes semi-static for other cohomology class $c^{\prime}$. In this case, we construct a new perturbation further so that the support of potential perturbation touches none of $\gamma^{ \pm}, \gamma^{ \pm}$if it is $c^{\prime}$-semi static for the perturbed Lagrangian. Since there are only four curves $\gamma^{ \pm}, \gamma^{\prime \pm}$ are concerned about, we can do it one by one. As these four curves are already in the Mañé sets containing that four Aubry classes, they do not lie in any other Mañé set, namely, any other Mañé 
set does not project surjectively on $\mathbb{T}^{2}$. Therefore, we complete the proof under the condition which will be proved in the following proposition.

We mention a result in opposite direction. In $T^{*} \mathbb{T}^{n}$, a Tonelli Hamiltonian without conjugate point is $C^{0}$-integrable [AABZ], the Riemannian tori are flat $[\mathrm{BI}]$. Next, let us verify that the condition of Theorem 3.1 is really generic.

Proposition 3.1. Assume the hypotheses $(\mathbf{H 1}, \mathbf{2})$ for the Lagrangian $L_{0}$ and assume $\mathcal{N}(c)=\mathbb{T}^{2}$ for some $c \in \partial \mathbb{F}_{0}$, then there exist $c$-semi static curves which approaches the fixed point in the direction of $\pm \Lambda_{2, x}$ as $t \rightarrow \infty$ or $t \rightarrow-\infty$.

Proof. First, let us study the case of $c \in \partial^{*} \mathbb{F}_{0}$. Other than the minimal measure supported on the fixed point, another minimal measure $\mu_{c}$ exists with non-zero rotation vector. For typical potential $V$, there exist at most three ergodic minimal measures for any first cohomology class $[\mathrm{BC}]$. So, in the universal covering space $\bar{\pi}: \mathbb{R}^{2} \rightarrow \mathbb{T}^{2}$, there exists a strip $\mathcal{S}_{c} \ni\{x=0\}$, bounded by two $c$-static curves $\xi_{c}$ and $\xi_{c}^{\prime}$ in the sense that $\bar{\pi} \xi_{c}, \bar{\pi} \xi_{c}^{\prime} \subset \mathcal{M}(c)$, such that int $\bar{\pi} \mathcal{S}_{c} \cap \mathcal{M}(c)=\{0\}$. If the Mañé set projects surjectively onto $\mathbb{T}^{2}$, this strip is filled with $c$-semi-static curves $\xi$ in the sense that $\bar{\pi} \xi$ is $c$ semi static, i.e. passing through every point in the strip there is at least one $c$-semi static curve.

Lemma 3.3. If the strip $\mathcal{S}_{c}$ is filled with $c$-semi-static curves and $\omega\left(\mu_{c}\right)$ is irrational, passing through each point in the strip there is only one semistatic curve.

Proof. As the configuration space is two-torus, any two orbits $\bar{\pi}\left(\xi_{c}, \dot{\xi}_{c}\right)$ and $\bar{\pi}\left(\xi_{c}^{\prime}, \dot{\xi}_{c}^{\prime}\right)$ share the same rotation vector, where we extend the standard projection $\bar{\pi}: \mathbb{R}^{2} \rightarrow \mathbb{T}^{2}$ in a natural way to $\bar{\pi}: T \mathbb{R}^{2} \rightarrow T \mathbb{T}^{2}$, it keeps the velocity unchanged. Properly choosing a section $\Sigma_{c}$ of $\mathbb{T}^{2}$ which is a circle, the closure of $\bar{\pi} \xi_{c} \cap \Sigma_{c}$ and of $\bar{\pi} \xi_{c}^{\prime} \cap \Sigma_{c}$ are Denjoy set. So, two curves $\bar{\pi} \xi_{c}$ and $\bar{\pi} \xi_{c}^{\prime}$ are in the same Aubry class, i.e. $h_{c}^{\infty}\left(x, x^{\prime}\right)+h_{c}^{\infty}\left(x^{\prime}, x\right)=0$ holds for any $x \in \bar{\pi} \xi_{c}, x^{\prime} \in \bar{\pi} \xi_{c}^{\prime}$.

If there were two semi-static curves $\gamma_{c}, \gamma_{c}^{\prime}$ intersecting each other at $\gamma_{c}(0)=\gamma_{c}^{\prime}(0)$, the $\omega$-limit set of the orbit $\left(\gamma_{c}, \dot{\gamma}_{c}\right)$ must be different from the $\omega$-limit set of the orbit $\left(\gamma_{c}^{\prime}, \dot{\gamma}_{c}^{\prime}\right)$. It is a consequence of the Lipschitz property of Aubry set. Let us assume $\gamma_{c}(t) \rightarrow 0$ and $\gamma_{c}^{\prime}(t) \rightarrow \bar{\pi} \xi_{c}$ as $t \rightarrow \infty$. There are four possibilities for $t \rightarrow-\infty$

1. $\gamma_{c}(t) \rightarrow\{0\}$ and $\gamma_{c}^{\prime}(t) \rightarrow \bar{\pi} \xi_{c}$ as $t \rightarrow-\infty$;

2. $\gamma_{c}(t) \rightarrow\{0\}$ and $\gamma_{c}^{\prime}(t) \rightarrow\{0\}$ as $t \rightarrow-\infty$; 
3. $\gamma_{c}(t) \rightarrow \bar{\pi} \xi_{c}$ and $\gamma_{c}^{\prime}(t) \rightarrow\{0\}$ as $t \rightarrow-\infty$;

4. $\gamma_{c}(t) \rightarrow \bar{\pi} \xi_{c}$ and $\gamma_{c}^{\prime}(t) \rightarrow \bar{\pi} \xi_{c}$ as $t \rightarrow-\infty$.

In Case 3, we join $\gamma_{c}(-\epsilon)$ and $\gamma_{c}^{\prime}(\epsilon)$ by a minimal curve $\xi$, join $\gamma_{c}^{\prime}(-\epsilon)$ and $\gamma_{c}(\epsilon)$ by a minimal curve $\xi^{\prime}$. The action along $\xi$ plus it along $\xi^{\prime}$ is smaller than the action along $\left.\gamma_{c}\right|_{[-\epsilon, \epsilon]}$ plus it along $\left.\gamma_{c}^{\prime}\right|_{[-\epsilon, \epsilon]}$. Notice $A\left(\left.\gamma_{c}\right|_{[-\epsilon, \epsilon]}\right)+$ $A\left(\left.\gamma_{c}^{\prime}\right|_{[-\epsilon, \epsilon]}\right)>A(\xi)+A\left(\xi^{\prime}\right)$ (the curve shortening lemma in Riemannian geometry). Because the curve $\left.\left.\gamma_{c}^{\prime}\right|_{(-\infty,-\epsilon]} * \xi^{\prime} * \gamma_{c}\right|_{[\epsilon, \infty)}$ is closed, along which the action is non-negative, the action along the curve $\left.\left.\gamma_{c}\right|_{\left[-K^{\prime},-\epsilon\right]} * \xi * \gamma_{c}^{\prime}\right|_{[\epsilon, K]}$ is smaller than the action along the curve $\left.\left.\gamma_{c}\right|_{\left[-K^{\prime}, 0\right]} * \gamma_{c}^{\prime}\right|_{[0, K]}$ for any large $K, K^{\prime}>0$. It implies that $h_{c}^{\infty}(x, 0)+h_{c}^{\infty}\left(0, x^{\prime}\right)>h^{\infty}\left(x, x^{\prime}\right)$ holds for any $x, x^{\prime} \in \bar{\pi} \xi_{c}$. It contradicts the assumption that the Mañe set covers the whole torus: for any $m \in \mathbb{T}^{2}, x, x^{\prime} \in \mathcal{M}(c)$ one has $h_{c}^{\infty}(x, m)+h_{c}^{\infty}\left(m, x^{\prime}\right)=$ $h^{\infty}\left(x, x^{\prime}\right)$.

In Case 4, there exists a semi-static curve $\gamma_{c}^{\prime \prime}$ which approaches $\{0\}$ as $t \rightarrow-\infty$ and approaches $\xi_{c}$ as $t \rightarrow \infty$. We join $\gamma_{c}^{\prime}(-\epsilon)$ to $\gamma_{c}(\epsilon)$ by a minimal curve $\xi^{\prime}$, join $\gamma_{c}(-\epsilon)$ to $\gamma_{c}^{\prime}(\epsilon)$ by a minimal curve $\xi$. Given $x, x^{\prime} \in \bar{\pi} \xi_{c}$, $\exists$ sequence $K_{i}, K_{i}^{\prime} \rightarrow \infty$ such that $\gamma_{c}^{\prime}\left(-K_{i}^{\prime}\right) \rightarrow x, \gamma_{c}^{\prime \prime}\left(K_{i}\right) \rightarrow x^{\prime}$. Also $\exists$ sequences $N_{i}, N_{i}^{\prime} \rightarrow \infty$ such that $\gamma_{c}\left(-N_{i}^{\prime}\right) \rightarrow x, \gamma_{c}^{\prime}\left(N_{i}\right) \rightarrow x^{\prime}$. As the action along $\gamma_{c}\left|\left[-N_{i}^{\prime},-\epsilon\right] * \xi * \gamma_{c}^{\prime}\right|_{\left[\epsilon, N_{i}\right]}$ plus the quantity $h_{c}^{\infty}\left(x^{\prime}, x\right)$ is non-negative, the action along $\left.\left.\left.\gamma_{c}^{\prime}\right|_{\left[-K_{i}^{\prime},-\epsilon\right]} * \xi^{\prime} * \gamma_{c}\right|_{[\epsilon, \infty)} * \gamma_{c}^{\prime \prime}\right|_{\left(-\infty, K_{i}\right]}$ is smaller than the action along $\left.\left.\gamma_{c}\right|_{\left.\left[-N_{i}^{\prime}, \infty\right)\right]} * \gamma_{c}^{\prime \prime}\right|_{\left(-\infty, K_{i}\right.}$. Again, it implies that $h_{c}^{\infty}(x, 0)+h_{c}^{\infty}\left(0, x^{\prime}\right)>$ $h^{\infty}\left(x, x^{\prime}\right)$ holds for $x, x^{\prime} \in \bar{\pi} \xi_{c}$. It is absurd. Other cases can be proved similarly.

Next, let us study the case $c \in \partial^{*} \mathbb{F}_{0}$ with rational $\omega\left(\mu_{c}\right)$. In this case, the strip $\mathcal{S}_{c}$ is bounded by two curves $\bar{\pi} \xi_{c}$ and $\bar{\pi} \xi_{c}^{\prime}$. In typical case, $\xi_{c}=\xi_{c}^{\prime}$ and there exists an edge $\mathbb{E}_{g} \subset \partial \mathbb{F}_{0}$ such that, for each $c \in \mathbb{E}_{g}$, the Mather set $\mathcal{M}(c)$ is made up by the fixed point and the curve $\xi_{g}$, see Figure 1. In this case one has $\bar{\pi} \mathcal{S}_{c}=\mathbb{T}^{2}$. Using the same argument to prove Lemma 3.3, one also has

Lemma 3.4. If the Mather set $\mathcal{M}(c)$ consists of a fixed point and a closed curve $\xi_{c}, \mathcal{N}(c)=\mathbb{T}^{2}$, then, passing through each point on $\mathbb{T}^{2}$ there is only one semi-static curve.

For $c \in \partial \mathbb{F}_{0} \backslash \partial^{*} \mathbb{F}_{0}$, the Mañé set is the same as the Aubry set. Therefore, passing through each point on $\mathbb{T}^{2}$ there is exactly one semi-static curve when $\mathcal{N}(c)=\mathbb{T}^{2}$.

As the second step of the proof of the proposition, let us study what will happen if the strip $\mathcal{S}_{c}$ is filled with semi-static curves. As the curves $\xi_{c}$ as well as $\xi_{c}^{\prime}$ is disjoint with the origin, some number $\delta>0$ exists so that these two curves do not hit the ball $B_{\delta}(0)$. Let $I_{c, \delta}^{ \pm} \subset \partial B_{\delta}(0)$ be such a set that 
passing through each point $x \in I_{c, \delta}^{ \pm}$the $c$-semi static curve approaches to the origin, as $t \rightarrow \pm \infty$. Obviously, the set $I_{c, \delta}^{ \pm} \neq \varnothing$ is closed and $I_{c, \delta}^{-} \cap I_{c, \delta}^{+}=\varnothing$ (see the proof of Lemma 3.3). Indeed, one has even stronger property as follows:

Lemma 3.5. Assume the Mañé set covers a neighborhood of the hyperbolic fixed point. Then, some number $\nu>0$ exists so that for suitably small $\delta>0$, the distance between $I_{c, \delta}^{+}$and $I_{c, \delta}^{-}$is not smaller than $\nu \delta$.

Proof. Let $x_{c, \delta}^{-} \in I_{c, \delta}^{-}$and $x_{c, \delta}^{+} \in I_{c, \delta}^{+}$be the endpoint of $I_{c, \delta}^{-}$and $I_{c, \delta}^{+}$respectively so that $d\left(x_{c, \delta}^{-}, x_{c, \delta}^{+}\right)=\nu \delta$. Passing through the point $x_{c, \delta}^{ \pm}$there is a $c$-semi static curve $\gamma_{c}^{ \pm}$such that $\gamma_{c}^{ \pm}(t) \rightarrow 0$ as $t \rightarrow \pm \infty$. One then has a wedge-shaped region in $B_{\delta}(0)$, bounded by $\gamma_{c}^{-}$and $\gamma_{c}^{+}$and denoted by $\mathbb{W}$, see the left of Figure 4. No semi-static curve passes through int $\mathbb{W}$ to approach the origin. Since the fixed point is hyperbolic, it has its stable and unstable manifold, determined by the generating functions $U^{+}$and $U^{-}$respectively, namely, the stable (unstable) manifold is the graph of the differential of $U^{+}$ $\left(U^{-}\right)$. Restricted in $\mathbb{W}$, these functions satisfy the condition

$$
U^{-}(x)-U^{-}(0) \geq \frac{\lambda_{1}^{2}}{3}\|x\|^{2}, \quad U^{+}(0)-U^{+}(x) \geq \frac{\lambda_{1}^{2}}{3}\|x\|^{2}, \quad \forall\|x\| \leq \delta .
$$

Let $\left\{x_{c, i, \delta}^{+}\right\} \in \partial B_{\delta}(0)$ be a sequence of points which are located between $x_{c, \delta}^{+}$and $x_{c, \delta}^{-}$so that $x_{c, i, \delta}^{+} \rightarrow x_{c, \delta}^{+}$as $i \rightarrow \infty$. By the assumption, passing through $x_{c, i, \delta}^{+}$there is a semi static curve $\gamma_{c, i}$ which keeps close to the curve $\gamma_{c, \delta}^{+}$before getting close to the origin. After that, it moves roughly along the curve $\gamma_{c, \delta}^{-}$and intersect the circle $\partial B_{\delta}(0)$ at a point $x_{c, i, \delta}^{-}$, see the left of Figure 4. Clearly, $x_{c, i, \delta}^{-} \rightarrow x_{c, \delta}^{-}$as $i \rightarrow \infty$. Up to a time translation, we
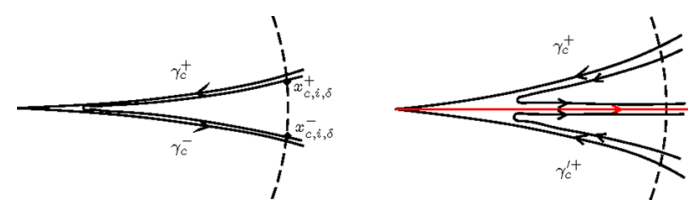

Figure 4: The red line is a semi-static curve departing from the origin.

assume $\gamma_{c, i}(0)=x_{c, i, \delta}^{+}, \gamma_{c, i}\left(T_{i}\right)=x_{c, i, \delta}^{-}$. If we set $U^{-}(0)=U^{+}(0)$, the action along the curve $\gamma_{c, i}$

$$
A\left[\left.\gamma_{c, i}\right|_{\left[0, T_{i}\right]}\right] \rightarrow U^{-}\left(x_{c, \delta}^{-}\right)-U^{+}\left(x_{c, \delta}^{+}\right) \geq \frac{2 \lambda_{1}^{2}}{3} \delta^{2}, \quad \text { as } i \rightarrow \infty .
$$


On the other hand, we connect the point $x_{c, i, \delta}^{-}$to $x_{c, i, \delta}^{+}$by a straight line $\zeta$ : $[0, \tau] \rightarrow M$, then $|\dot{\zeta}| \leq \frac{\nu \delta}{\tau}$. Since $L=\frac{1}{2}\left\langle A^{-1} \dot{x}, \dot{x}\right\rangle+V(x)$ there exists some constant $a_{1}>0$ which is independent of $\delta$ such that $L(\zeta, \dot{\zeta}) \leq a_{1}\left(\left(\frac{\nu \delta}{\tau}\right)^{2}+\delta^{2}\right)$. Let $\tau=\nu$, then one has

$$
A\left[\left.\zeta\right|_{[0, \nu]}\right] \leq 2 a_{1} \nu \delta^{2} .
$$

If $\nu<\frac{\lambda_{1}^{2}}{3 a_{1}}$, one would have $A\left[\left.\zeta\right|_{[0, \nu]}\right]<A\left[\left.\gamma_{c, i}\right|_{\left[0, T_{i}\right]}\right]$ for sufficiently large $i$. It is absurd since the curve $\gamma_{c, i}$ is assumed to be semi-static.

Lemma 3.6. Assume the Mañé set for a class c covers a neighborhood of the hyperbolic fixed point. Let $\gamma_{c}^{+}$and $\gamma_{c}^{-}$be c-semi static curve passing through $I_{c, \delta}^{+}$and $I_{c, \delta}^{-}$respectively, if both $\gamma_{c}^{+}$and $\gamma_{c}^{-}$approach the origin in the direction of $\Lambda_{1, x}$ as $t \rightarrow \pm \infty$, then, they approach it in opposite direction, i.e.

$$
\lim _{t \rightarrow \infty} \frac{\dot{\gamma}_{c}^{+}(t)}{\left\|\dot{\gamma}_{c}^{+}(t)\right\|}=-\lim _{t \rightarrow-\infty} \frac{\dot{\gamma}_{c}^{-}(t)}{\left\|\dot{\gamma}_{c}^{-}(t)\right\|} ;
$$

Proof. If both curves $\gamma_{c}^{+}$and $\gamma_{c}^{-}$approach the origin in the direction of $\Lambda_{1, x}$ such that

$$
\lim _{t \rightarrow \infty} \frac{\dot{\gamma}_{c}^{+}(t)}{\left\|\dot{\gamma}_{c}^{+}(t)\right\|}=\lim _{t \rightarrow-\infty} \frac{\dot{\gamma}_{c}^{-}(t)}{\left\|\dot{\gamma}_{c}^{-}(t)\right\|},
$$

there would be a sharp wedge-shaped region in $B_{\delta}(0)$, bounded by $\gamma_{c}^{+}$and $\gamma_{c}^{-}$, with a vertex at the origin and denoted $\mathbb{W}$. None of semi static curves passes through $\mathbb{W}$ to approach the origin. The rest of the proof is applying Lemma 3.5.

Next, let us study the case that both $I_{c, \delta}^{-}$and $I_{c, \delta}^{+}$are connected. Denote by $x_{c, \delta}^{+}, x_{c, \delta}^{+}$and $x_{c, \delta}^{-}, x_{c, \delta}^{--}$the end points of $I_{c, \delta}^{+}$and $I_{c, \delta}^{-}$respectively. $I_{c, \delta}^{ \pm}$is a point if $x_{c, \delta}^{ \pm}=x_{c, \delta}^{\prime \pm}$. Passing through a point $x_{c, i, \delta}^{+} \in \partial B_{\delta}(0) \backslash I_{c, \delta}^{+}$, close to $I_{c, \delta}^{+}$, there is a $c$-semi static curve $\gamma_{c, i}$ which will get close to the origin and eventually depart from the disk $B_{\delta}(0)$. Let $x_{c, i, \delta}^{+}, x_{c, i, \delta}^{\prime+} \in \partial B_{\delta}(0)$ be two sequence of points approaching $I_{c, \delta}^{+}$from different sides, $x_{c, i, \delta}^{+} \rightarrow x_{c, \delta}^{+}$and $x_{c, i, \delta}^{\prime+} \rightarrow x_{c, \delta}^{\prime+}$. Let $\gamma_{c, i}$ and $\gamma_{c, i}^{\prime}$ be the semi static curves passing through the points $x_{c, i, \delta}^{+}$and $x_{c, i, \delta}^{\prime+}$ respectively, they shall intersect the circle $\partial B_{\delta}(0)$ at points $x_{c, i, \delta}^{-}$and $x_{c, i, \delta}^{\prime-}$ when they are going to leave the disk. Some points $x_{c, \delta}^{-}, x_{c, \delta}^{--} \in \partial B_{\delta}(0)$ exist such that $x_{c, i, \delta}^{-} \rightarrow x_{c, \delta}^{-}$and $x_{c, i, \delta}^{\prime-} \rightarrow x_{c, \delta}^{\prime-}$ as $i \rightarrow \infty$.

If $x_{c, \delta}^{-} \neq x_{c, \delta}^{-}$, they divide the circle $\partial B_{\delta}(0)$ into two arcs. The arc not containing $I_{c, \delta}^{+}$is nothing else but $I_{c, \delta}^{-}$. Indeed, by a time translation, certain $T_{i}^{ \pm}>0$ exists such that $\gamma_{c, i}\left(-T_{i}^{+}\right)=x_{c, i, \delta}^{+}, \gamma_{c, i}\left(T_{i}^{-}\right)=x_{c, i, \delta}^{-}$and 
$d\left(\gamma_{c, i}(0), 0\right)=\min _{t \in\left[-T_{i}^{+}, T_{i}^{-}\right]} d\left(\gamma_{c, i}(t), 0\right)$. When $x_{c, i, \delta}^{-} \rightarrow x_{c, \delta}^{-}$, one has $T_{i}^{-} \rightarrow$ $\infty$. It follows that both $x_{c, \delta}^{-}$and $x_{c, \delta}^{\prime-}$ are in $I_{c, \delta}^{-}$. As it is connected, the arc is exactly the set $I_{c, \delta}^{-}$itself. If $x_{c, \delta}^{-}=x_{c, \delta}^{\prime}, I_{c, \delta}^{-}$is just a point.

Recall four points $\left(x_{\delta}^{+}, x_{\delta}^{\prime+}, x_{\delta}^{-}, x_{\delta}^{\prime-}\right)$ defined before: there are exactly four orbits $\left(\gamma^{ \pm}, \dot{\gamma}^{ \pm}\right),\left(\gamma^{\prime \pm}, \dot{\gamma}^{\prime \pm}\right)$ intersecting the circle $\partial B_{\delta}(0)$ at these four points such that the formulae (3.8) (3.9) hold for $\gamma^{ \pm}, \gamma^{\prime \pm}$. Let us consider the location of $x_{c, \delta}^{+}$and $x_{c, \delta}^{\prime+}$ with respect to the points $x_{\delta}^{+}$and $x_{\delta}^{\prime+}$. All possibilities are listed below:

1. the two-point set $\left\{x_{c, \delta}^{+}, x_{c, \delta}^{++}\right\}$does not overlap the set $\left\{x_{\delta}^{+}, x_{\delta}^{\prime+}\right\}$;

2. the set $\left\{x_{c, \delta}^{+}, x_{c, \delta}^{\prime+}\right\}$ overlaps the set $\left\{x_{\delta}^{+}, x_{\delta}^{\prime+}\right\}$.

The following property is guaranteed by the condition $\lambda_{1}<\lambda_{2}$ : by shrinking the size of $\delta$, the direction of $x_{c, \delta}^{+}\left(x_{c, \delta}^{\prime+}\right)$ is not close to the direction of $\pm \Lambda_{2, x}$ if it is neither the point $x_{\delta}^{+}$nor the point $x_{\delta}^{\prime+}$ itself.

For Case 1, because of Lemma 3.6, the semi static curves passing through $x_{c, \delta}^{+}$and passing through $x_{c, \delta}^{++}$approach the origin in the same direction. By applying Lemma 3.2, one sees that, when $x_{c, i, \delta}^{+}$approaches $x_{c, \delta}^{+}$from one side of $I_{c, \delta}^{+}$, the semi-static curve $\gamma_{c, i}$ will depart from the disk $B_{\delta}(0)$ in a direction close to $\Lambda_{2, x}$; the semi-static curve $\gamma_{c, i}^{\prime}$ will depart from the disk $B_{\delta}(0)$ in a direction close to $-\Lambda_{2, x}$ when $x_{c, i, \delta}^{\prime+}$ approaches $x_{c, \delta}^{++}$from another side of $I_{c, \delta}^{+}$. So, the direction of $x_{c, i, \delta}^{-}$is nearly opposite to the direction of $x_{c, i, \delta}^{\prime-}$ if $i \rightarrow \infty$. In this case, we claim that $\left\{x_{c, \delta}^{-}, x_{c, \delta}^{\prime-}\right\}=\left\{x_{\delta}^{-}, x_{\delta}^{\prime-}\right\}$. To see it, let us shrink the radius $\delta$ to a smaller $\delta^{\prime}$. The curve $\gamma_{c, i}$ will intersect the circle $\partial B_{\delta^{\prime}}(0)$ at a point $x_{c, i, \delta^{\prime}}^{-}$when it is going to leave the disk $B_{\delta^{\prime}}(0)$. If $x_{c, \delta}^{-} \notin\left\{x_{\delta}^{-}, x_{\delta}^{-}\right\}$, the direction of $x_{c, i, \delta^{\prime}}^{-}$would not be close to the direction of $\pm \Lambda_{2, x}$ if $\delta^{\prime}$ is sufficiently small and $i$ is sufficiently large. Along the curve we retreat from the point $x_{c, i, \delta^{\prime}}^{-}$to the point $x_{c, i, \delta^{\prime}}^{+}$where the curve enters the disk, it is guaranteed by Lemma 3.2 that the direction of $x_{c, i, \delta^{\prime}}^{+}$, and consequently, the direction of $x_{c, \delta}^{+}$is close to the direction of $\pm \Lambda_{2, x}$. It is obviously absurd. For the sequence $\left\{x_{c, i, \delta}^{\prime-}\right\}$ one has the same conclusion. Therefore, the set $I_{c, \delta}^{-}$occupies an arc with length close to $\pi \delta$. Because the fixed point is hyperbolic, semi static curves crossing the arc $I_{c, \delta}^{-}$produces orbits staying on the unstable manifold. Therefore, some number $d>0$ exists, no matter how small the number $\delta>0$ could be, such that the circle $\partial B_{d}(0)$ contains an arc $I_{c, d}^{-}$with length close to $\pi d$. Through each point of $I_{c, d}^{-}$there is a semi static curve which approaches the origin as the time approaches minus infinity, see the left figure in Figure 5. The figure in 


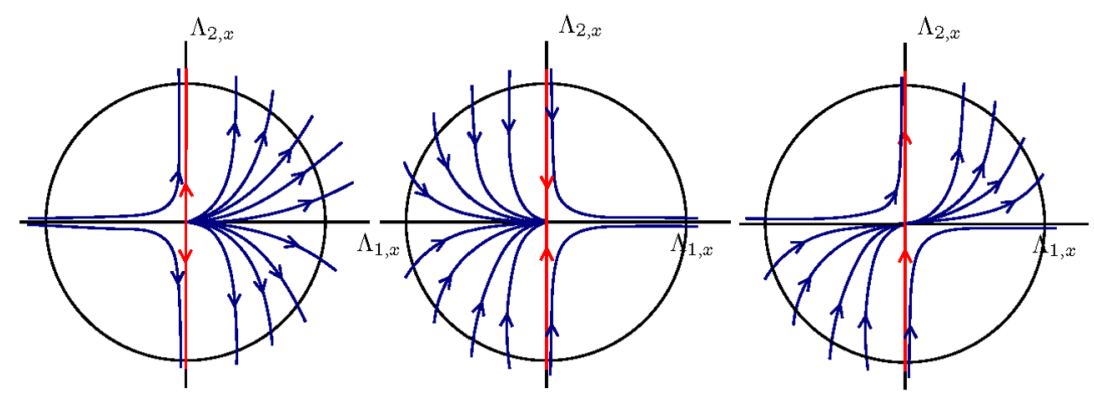

Figure 5: The red lines are the semi-static curves approach the origin along the direction of $\Lambda_{2, x}$, as $t \rightarrow \pm \infty$.

the middle corresponds to case that the two-point set $\left\{x_{c, \delta}^{-}, x_{c, \delta}^{\prime-}\right\}$ does not overlap the set $\left\{x_{\delta}^{-}, x_{\delta}^{\prime-}\right\}$. In both cases, there are $c$-semi static curves which approaches the origin in the direction of $\Lambda_{2, x}$ as $t \rightarrow-\infty$ or as $t \rightarrow \infty$. The figure on the right is in the case when the set $\left\{x_{c, \delta}^{+}, x_{c, \delta}^{\prime+}\right\}$ overlaps the set $\left\{x_{\delta}^{+}, x_{\delta}^{\prime+}\right\}$, namely, the case 2 .

It follows from the argument above that each point $x_{c, \delta}^{+} \in \partial I_{c, \delta}^{+} \backslash\left\{x_{\delta}^{+}, x_{\delta}^{\prime+}\right\}$ is associated with a point of $\left\{x_{\delta}^{-}, x_{\delta}^{--}\right\} \cap I_{c, \delta}^{-}$, a non-empty set, the circle $\partial B_{\delta}(0)$ is divided into two arcs by these two points. One of the arcs does not contain points of $I_{c, \delta}^{+} \cup I_{c, \delta}^{-}$except for the end points. Consequently, $I_{c, \delta}^{ \pm}$ contains at most two connected components; and if $I_{c, \delta}^{+}$has two connected components, then so does $I_{c, \delta}^{-}$.

Let $\gamma_{c}^{+}, \gamma_{c}^{\prime+}$ be the semi-static curve passing through different connected components of $I_{c, \delta}^{+}$. If $\gamma_{c}^{+}$approach the origin in the direction of $\pm \Lambda_{i, x}$, then $\gamma_{c}^{\prime+}$ approaches in the direction of $\mp \Lambda_{i, x}$ :

$$
\lim _{t \rightarrow \infty} \frac{\dot{\gamma}_{c}^{+}(t)}{\left\|\dot{\gamma}_{c}^{+}(t)\right\|}=-\lim _{t \rightarrow \infty} \frac{\dot{\gamma}_{c}^{+}(t)}{\left\|\dot{\gamma}_{c}^{+}(t)\right\|} .
$$

If not, there would be a sharp wedge-shaped region $\mathbb{W}$ in $B_{\delta}(0)$, bounded by $\gamma_{c}^{+}$and $\gamma_{c}^{\prime+}$ with a vertex at the origin such that through each point in the interior of $\mathbb{W}$, there is a semi-static curve which does not approach the origin as $t \rightarrow \infty$. As these semi-static curves do not intersect each other, both $\gamma_{c}^{+}$ and $\gamma_{c}^{\prime+}$ approach the origin as $t \rightarrow \infty$, there must be a semi static curve which passes through $\mathbb{W}$ and approaches the origin as $t \rightarrow-\infty$, see the right of Figure 4. This contradicts Lemma 3.4. If $\gamma_{c}^{+}, \gamma_{c}^{\prime+}$ approach the fixed point in the direction of $\Lambda_{1, x}$, then $\left\{x_{\delta}^{-}, x_{\delta}^{\prime-}\right\}=I_{c, \delta}^{-}$, i.e. there exist semi-static 
curve approaching the fixed point in the direction of $\Lambda_{2, x}$ as $t \rightarrow \pm \infty$. This completes the proof of Proposition 3.1.

It holds unconditionally that $\mathcal{N}(c) \subsetneq \mathbb{T}^{2}$ if $c \in \operatorname{int} \mathbb{E}_{i} \subset \partial \mathbb{F}_{0} \backslash \partial^{*} \mathbb{F}_{0}$, since one has $\mathcal{N}(c)=\mathcal{A}(c) \subsetneq \mathcal{A}\left(c^{\prime}\right)$ for $c \in \operatorname{int} \mathbb{E}_{i}$ and $c^{\prime} \in \partial \mathbb{E}_{i}$. It also holds unconditionally that $\mathcal{N}(c) \subsetneq \mathbb{T}^{2}$ if $c \in \operatorname{int} \mathbb{E}_{i} \subset \partial^{*} \mathbb{F}_{0}$ if $\mathcal{M}(c)$ consists of the fixed point and a periodic curve.

In the latter case, if $\mathcal{N}(c)=\mathbb{T}^{2}$, one can see from the proof of Lemma 3.4 that $\mathcal{N}(c)=\mathcal{A}(c)$. But it is impossible since $\mathcal{A}(c) \subsetneq \mathcal{A}\left(c^{\prime}\right)$ for $c^{\prime} \in \partial \mathbb{E}_{i}$. A typical portrait of semi-static curves is shown in Figure 1.

In the former case, let $\mathbb{E}_{i} \subset \partial \mathbb{F}_{0} \backslash \partial^{*} \mathbb{F}_{0}$ be an edge joined to other two edges at the vertex $c_{i}, c_{i+1}$ respectively. By Theorem 2.1, the Aubry set for $c_{j}$ consists of two minimal homoclinic curves $\gamma_{j-1}$ and $\gamma_{j}$ with $j=i, i+1$. Denote by $g_{j} \in \mathbb{Z}^{2}$ the homology class of $\gamma_{j}$, then the matrix $\left(g_{j-1}, g_{j}\right)$ is uni-module. By introducing suitable coordinates on $\mathbb{T}^{2}$, we can assume

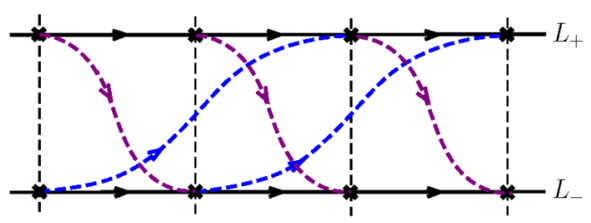

$g_{i}=(1,0)$. In this coordinate system, $g_{i-1}=(k, 1)$ and $g_{i+1}=\left(k^{\prime},-1\right)$. In this figure, each unit square represents a fundamental domain of $\mathbb{T}^{2}$ in the universal covering space, the horizontal line represents the lift of the homoclinic curve $\gamma_{i}$, which stays in the Aubry set for each $c \in \mathbb{E}_{i}$. The blue dashed lines represent the lift of the $\gamma_{i-1}$ which stays in the Aubry set for the class at one end-point of $\mathbb{E}_{i}$. The purple dashed lines represents the lift of the $\gamma_{i+1}$ which stays in the Aubry set for the class at another end-point of $\mathbb{E}_{i}$.

\subsection{Annulus of cohomology equivalence around the disk $\mathbb{F}_{0}$}

To establish the new version of cohomology equivalence around the flat $\mathbb{F}_{0}$, we make use of the upper semi-continuity of Mañé set in the first cohomology class. According to Theorem 3.1, for $C^{2+}$-generic potential $V$ in the Lagrangian $L_{0}$, it holds simultaneously for all $c \in \partial \mathbb{F}_{0}$ that the Mañé set does not cover the configuration space: $\mathcal{N}(c) \subsetneq \mathbb{T}^{2}$. As $\partial \mathbb{F}_{0}$ is compact, certain $\Delta_{V}>0$ exists such that for each $c \in \alpha_{0}^{-1}(\Delta)$ with $\Delta \leq 2 \Delta_{V}$, the Mañé set does not project surjectively on the 2-torus too. Recall $\alpha_{0}$ denotes the $\alpha$-function for $L_{0}$.

Since the Lagrangian $L_{0}$ is defined on 2-torus, for each average action $\Delta>\min \alpha_{0}$, the dynamics on the energy level $G^{-1}(\Delta)$ is similar to an area- 
preserving twist map, where $G=\frac{1}{2}\left\langle A^{-1} y, y\right\rangle-V(x)$. First of all, the rotation vector of each minimal measure is not zero. Thus, any minimal measure is not supported on fixed points. Next, for any class $c \in \alpha_{0}^{-1}(\Delta)$, all $c$-minimal measures share the same rotation direction. Otherwise, the Lipschitz graph property of Mather set will be violated. Each ergodic minimal measure is supported on a periodic orbit if the rotation direction is rational.

Therefore, $\forall c \in \alpha_{0}^{-1}(\Delta)$, some circle $S_{c} \subset \mathbb{T}^{2}$ exists so that each semistatic curve intersects the circle transversally and $\mathcal{N}(c) \cap S_{c} \subsetneq S_{c}$. As Mañé set is closed, there exist finitely many intervals $I_{c, i} \subset S_{c}$ disjoint to each other such that $\mathcal{N}(c) \cap S_{c} \subset \cup I_{c, i}$, see the left figure in Figure 3. To establish cohomology equivalence, we work in the extended configuration space $\mathbb{T}^{3}$ where the extra dimension is for the time $t$.

In the extended configuration space $\mathbb{T}^{3}$, we choose a section $\Sigma_{c}=S_{c} \times \mathbb{T}$. As $L_{0}$ is independent of $t$, the Mañé set in the extended space, denoted by $\mathcal{N}_{L_{0}}(c)$, stays in the strips: $\mathcal{N}_{L_{0}}(c) \cap \Sigma_{c} \subset\left(\cup I_{c, i}\right) \times \mathbb{T}$, see the figure in middle of Figure 3.

Recall the Lagrangian $L_{0}$ is a truncation of the Lagrangian $L_{\epsilon}$ of (1.1) rewritten as the following

$$
L_{\epsilon}=\frac{1}{2}\langle A \dot{x}, \dot{x}\rangle+V(x)+\sqrt{\epsilon} R_{\epsilon}(x, \dot{x}, t), \quad(x, y) \in \mathbb{T}^{2} \times \mathbb{R}^{2} .
$$

Let $\mathbb{F}_{\epsilon}=\left\{c \in H^{1}\left(\mathbb{T}^{2}, \mathbb{R}\right): \alpha_{\epsilon}(c)=\min \alpha_{\epsilon}\right\}$. Because of the upper-semi continuity of Mañé set with respect to small perturbation of Lagrangian, for each $c \in \partial \mathbb{F}_{\epsilon}$ there is $\epsilon_{c}>0$ such that for each $\epsilon \leq \epsilon_{c}$ one has

$$
\mathcal{N}_{L_{\epsilon}}(c) \cap \Sigma_{c} \subset\left(\cup I_{c, i}\right) \times \mathbb{T},
$$

see the right figure of Figure 3.

Because $\partial \mathbb{F}_{\epsilon}$, the boundary of $\mathbb{F}_{\epsilon}$, is compact, some $\epsilon_{V}>0$ exists such that the relation (3.12) holds simultaneously for any $c \in \partial \mathbb{F}$ and any $\epsilon \leq \epsilon_{V}$. Applying the upper semi-continuity of Mañé set with respect to cohomology class again, one obtains that Formula (3.12) holds for any $c$ such that $\alpha_{\epsilon}(c) \in$ $\left(\min \alpha_{\epsilon}, \min \alpha_{\epsilon}+\Delta_{V}\right)$. Therefore, one has

Theorem 3.2. Each $C^{2+}$-generic potential $V$ in (3.11) is associated with positive constants $\epsilon_{V}, \Delta_{V}>0$ such that for each $\epsilon \in\left[0, \epsilon_{V}\right]$ some annulus

$$
\mathbb{A}_{\epsilon}=\left\{c \in H^{1}\left(\mathbb{T}^{2}, \mathbb{R}\right): \alpha_{\epsilon}(c) \in\left(\min \alpha_{\epsilon}, \Delta_{V}\right)\right\}
$$

admits a foliation of circles $\Gamma_{E}=\left\{c: \alpha_{\epsilon}(c)=E\right\}$, each circle establishes a relation of cohomology equivalence. 
Proof. We choose the section $\Sigma_{c}=S_{c} \times \mathbb{T}$ and obtain from (3.12) that

$$
V_{c}=\operatorname{span}\{(0,0,1)\}, \quad \forall c \in \alpha_{\epsilon}^{-1}\left(\min \alpha_{\epsilon}, \Delta_{V}\right) \text {. }
$$

In this case, one has $V_{c}^{\perp}=\operatorname{span}\{(1,0,0),(0,1,0)\}$. For any two classes $c$, $c^{\prime}$ on the same circle $\Gamma_{E}$, one has $\left(c, \alpha_{\epsilon}(c)\right)-\left(c^{\prime}, \alpha_{\epsilon}\left(c^{\prime}\right)\right)=\left(c-c^{\prime}, 0\right) \in V_{c}^{\perp}$, namely, if two classes are located on the same circle, they are cohomologically equivalent.

Theorem 1.1 follows immediately from this theorem. A special case of the theorem is $\epsilon=0$, when it appears to be a classical system with two degrees of freedom, far from integrable. By this theorem, one is able to show the topological transitivity among all Aubry sets $\tilde{\mathcal{A}}(c)$ on any energy level set where $\alpha_{0}(c)-\min \alpha_{0}>0$ is small. The proof will be finished later.

By this theorem, one also obtains the cohomology equivalence in Tonelli Lagrangian with three degrees of freedom. Restricted on energy level set, a Hamiltonian with $n$-degrees of freedom can be reduced to a Hamiltonian with $n-\frac{1}{2}$ degrees of freedom. Let us study what is the relation between the $\alpha$-function of the original autonomous Hamiltonian and the reduced Hamiltonian.

Theorem 3.3. For the Hamiltonian $H\left(x, x_{n}, y, y_{n}\right)$, we assume that $\partial_{y_{n}} H \neq$ 0 holds on $\left(\mathbb{T}^{n} \times B\right) \cap\left\{H^{-1}(E)\right\}$ where $E>\min \alpha_{H}, B \subset \mathbb{R}^{n}$ is a ball. Let $y_{n}=-\lambda G(x, y, t)$ be the solution of $H\left(x, \frac{1}{\lambda} t, y,-\lambda G\right)=E$, let $\alpha_{H}, \alpha_{G}$ be the $\alpha$-function for the Lagrangian $L_{H}$ and $L_{G}$, determined by $H$ and $G$ respectively. For a class $c \in H^{1}\left(\mathbb{T}^{n-1}, \mathbb{R}\right)$, if the c-minimal curve $x(t)$ of $L_{G}$ satisfies the condition

$$
\left(x(t), \lambda^{-1} t, y(t),-\lambda G(x(t), y(t), t)\right) \in \mathbb{T}^{n} \times B, \quad \forall t \in \mathbb{R}
$$

where $y(t)=\partial_{\dot{x}} L_{G}(x(t), \dot{x}(t), t)$, then one has $\left(c,-\lambda \alpha_{G}(c)\right) \in \alpha_{H}^{-1}(E)$.

Proof. Let $\tilde{c}=\left(c,-\lambda \alpha_{G}(c)\right), \tilde{\gamma}=\left(\gamma, \gamma_{n}\right), \tilde{x}=\left(x, x_{n}\right)$ and $\tilde{y}=\left(y, y_{n}\right)$. Let $\gamma$ be $c$-minimal curve for the Lagrange flow $\phi_{L_{G}}^{t}, \tilde{\gamma}$ is then $\tilde{c}$-minimal curve for the Lagrange flow $\phi_{L_{\tilde{G}}}^{t}$ if $\gamma_{n}=x_{n}$ and $\tilde{\gamma}$ is re-parameterized $\tau \rightarrow t$. If $x=x(\tau)$ is a solution of $\phi_{L_{G}}^{t}$, one obtains $y=y(\tau)$ from the Hamiltonian equations. As $H(\tilde{x}(t), \tilde{y}(t)) \equiv E$, we find

$$
\begin{aligned}
{\left[A_{G}(\gamma)\right] } & =\int\left(\left\langle\frac{d x}{d \tau}, y-c\right\rangle-G+\alpha_{G}(c)\right) d \tau \\
& =\int(\langle\dot{\tilde{x}}, \tilde{y}-\tilde{c}\rangle-H+E) d t=\left[A_{H}(\tilde{\gamma})\right] .
\end{aligned}
$$

This completes the proof. 
We restrict ourselves to the case $n=3$. Let $\pi_{3}: \mathbb{R}^{3} \rightarrow \mathbb{R}^{2}$ be the projection so that $\pi_{3} \tilde{x}=x$. According to this theorem, $\pi_{3}^{-1}: H^{1}\left(\mathbb{T}^{2}, \mathbb{R}\right) \rightarrow$ $\alpha_{H}^{-1}(E)$ is a homeomorphism when $c$ is restricted in $\mathbb{F}+d$, a $d$-neighborhood of $\mathbb{F}=\alpha_{G}^{-1}\left(\min \alpha_{G}\right)$.

Let $G_{\epsilon}$ be the Hamiltonian obtained from $L_{\epsilon}$ which is reduced from a Tonelli Hamiltonian $H_{\epsilon}\left(x, x_{3}, y, y_{3}\right)=E$. What obtained in Theorem 3.2 have their counterpart on the level set $\left\{\alpha_{H_{\epsilon}}^{-1}(E)\right\}$ where $\tilde{c} \in \pi_{3}^{-1}(\mathbb{F}+d) \cap$ $\alpha_{H_{\epsilon}}^{-1}(E)$. Therefore, restricted on $\alpha_{H_{\epsilon}}^{-1}(E)$ one establishes some cohomology equivalence provided $E>\min \alpha_{H_{\epsilon}}$.

Theorem 3.4. Each $C^{2+}{ }_{-}$generic potential $V$ in (1.1) is associated with a constant $\Delta_{V}>0$ such that an annulus-like surface

$$
\tilde{\mathbb{A}}_{\epsilon}=\alpha_{H_{\epsilon}}^{-1}(E) \cap\left\{c_{3} \in\left(\min \alpha_{\epsilon}, \min \alpha_{\epsilon}+\Delta_{V}\right)\right\}
$$

admits a foliation of contour circles of $\Gamma_{\lambda}=\left\{\tilde{c}: \alpha_{H_{\epsilon}}(\tilde{c})=E, c_{3}=\lambda\right\}$, each of these circles establishes a relation of cohomology equivalence.

\section{Generalized transition chain}

The goal of this section is to prove Theorem 1.2. The concept of transition chain was proposed by Arnold in [A64] for the construction of diffusion orbits. It was formulated in geometric language. The generalized transition chain formulated in our previous work [CY1, CY2, LC] is a combination of Arnold's mechanism and the mechanism of cohomology equivalence. It is in variational language which requires less information about the geometric structure.

\subsection{Genericity of the generalized transition chain}

By variational method, two types of orbit have been constructed to connect one Aubry set to another one nearby. One is based on Arnold's mechanism, the other is based on cohomology equivalence.

We recall Arnold's example in [A64]. If the stable and unstable manifold of a circle intersect transversally each other, the unstable manifold intersects the stable manifold of other circles nearby. To understand this phenomenon from variational point of view, let us work in a finite covering space $\check{\pi}$ : $\mathbb{T} \times 2 \mathbb{T} \rightarrow \mathbb{T}$ such that the lift of the circle consists of two copies $\left\{x_{2}=\right.$ $0\} \cup\left\{x_{2}=1\right\}$. In the covering space, the Aubry set consists of two circles, the Mañé set is composed by the Aubry set and the minimal heteroclinic orbit connecting the circles. The transversal intersection of the stable and unstable manifold implies that $\mathcal{N}_{0}(c) \backslash\left(\mathcal{A}_{0}(c)+\delta\right)$ consists of discrete points only. 
Hinted by this observation one obtains the variational version of Arnold's mechanism.

Let $\check{\pi}: \check{M} \rightarrow M$ be a finite covering of the space $M$, let $\mathcal{N}(c, \check{M})$ and $\mathcal{A}(c, \check{M})$ be the Mañé set, Aubry set with respect to $\check{M}$. The following condition HA (hypothesis of Arnold) ensures that the $\tilde{\mathcal{A}}(c)$ is dynamically connected to $\tilde{\mathcal{A}}\left(c^{\prime}\right)$ if $c^{\prime}$ is sufficiently close to $c$ and $\tilde{\mathcal{A}}\left(c^{\prime}\right)$ is contained in a small neighborhood of $\tilde{\mathcal{A}}(c)$

(HA): there exists a finite covering $\check{\pi}: \check{M} \rightarrow M$ such that

1. for time-periodic systems: $\check{\pi} \mathcal{N}_{0}(c, \check{M}) \backslash\left(\mathcal{A}_{0}(c, \check{M})+\delta^{\prime}\right)$ is non-empty and totally disconnected;

2. for autonomous systems: $\left.\check{\pi} \mathcal{N}(c, \check{M})\right|_{D} \backslash\left(\mathcal{A}(c, \check{M})+\delta^{\prime}\right)$ is non-empty and totally disconnected, where $D$ is a section of $\check{M}$.

It is not necessary to work always in nontrivial finite covering space. If the Aubry set contains more than one class, one can choose $\check{M}=M$. The condition (HA) appears weaker than the condition of transversal intersection of stable and unstable manifolds. It also works if the intersection is only topologically transversal.

Definition 4.1. Two cohomology classes $c, c^{\prime} \in H^{1}(M, \mathbb{R})$ are joined by a generalized transition chain if a continuous curve $\Gamma:[0,1] \rightarrow H^{1}(M, \mathbb{R})$ exists such that and for each $s \in[0,1]$ at least one of the following cases takes place:

1. the condition (HA) holds for $\Gamma(s)$ and $\mathcal{A}\left(\Gamma\left(s^{\prime}\right)\right)$ lies in a small neighborhood of $\mathcal{A}(\Gamma(s))$ if $\left|s^{\prime}-s\right|$ is small;

2. there is $\delta_{s}>0$, for each $s^{\prime} \in\left(s-\delta_{s}, s+\delta_{s}\right), \Gamma\left(s^{\prime}\right)$ is cohomologically equivalent to $\Gamma(s)$.

If the Lagrangian is autonomous, it is also required that $\alpha(\Gamma(s))$ keeps constant.

By the definition, for each cohomology class $\Gamma(s)$, the Aubry set $\tilde{\mathcal{A}}(\Gamma(s))$ can be connected to certain Aubry set $\tilde{\mathcal{A}}\left(\Gamma\left(s^{\prime}\right)\right)$ nearby, either by Arnold's mechanism which looks like heteroclinic orbits as shown in the first case, or by cohomology equivalence. The existence of generalized transition chain implies the existence of sequence of local connecting orbits $\left(\gamma_{i}, \dot{\gamma}_{i}\right)$, a sequence of numbers $s_{i}$ such that $\alpha\left(\gamma_{i}, \dot{\gamma}_{i}\right) \subset \mathcal{A}\left(\Gamma\left(s_{i}\right)\right)$ and $\left.\omega\left(\gamma_{i}, \dot{\gamma}_{i}\right)\right) \subset \mathcal{A}\left(\Gamma\left(s_{i+1}\right)\right)$. Global connecting orbits are constructed shadowing these local connecting orbits, one can refer [CY2, LC] for the details.

To establish a transition chain for the problem under consideration, let us consider what candidates of transition chain we already have. 
Given an irreducible class $g$, let $\nu_{g}<\nu_{g}^{h}$ such that $\alpha_{\epsilon}\left(\nu_{g} g\right)<\Delta_{V}$ and $\alpha_{\epsilon}\left(\nu_{g}^{h} g\right)=\epsilon^{2 \kappa-1}$ where $\kappa \in\left(0, \frac{1}{2}\right)$. We consider the set

$$
\mathbb{C}_{g, \nu_{g}, \nu_{g}^{h}}=\bigcup_{\nu \in\left[\nu_{g}, \nu_{g}^{h}\right]} \mathscr{L}_{\beta_{\epsilon}}(\nu g) .
$$

According to Theorem 1.1 in [C15], for $C^{5+}$-generic potential $V$ in the Lagrangian $L_{\epsilon}$ of (3.11), the set $\mathbb{C}_{g, \nu_{g}, \nu_{g}^{h}}$ looks like a channel. For each class $c$ in the channel, the Aubry set lies on some normally hyperbolic invariant cylinder. Because the thickness of the annulus $\mathbb{A}_{\epsilon}$ is of order $\sqrt{\Delta_{V}}>0$, independent of $\epsilon$, one has

Theorem 4.1 (Overlap Property). Given any two irreducible classes $g, g^{\prime} \in$ $H_{1}\left(\mathbb{T}^{2}, \mathbb{Z}\right)$, there exists a positive number $\epsilon_{V}=\epsilon_{V}\left(g, g^{\prime}\right)>0$ such that the channels intersect the annulus: $\mathbb{C}_{g, \nu_{g}, \nu_{g}^{h}} \cap \mathbb{A}_{\epsilon} \neq \varnothing$ and $\mathbb{C}_{g^{\prime}, \nu_{g^{\prime}}, \nu_{g^{\prime}}^{h}} \cap \mathbb{A}_{\epsilon} \neq \varnothing$ provided $0<\epsilon \leq \epsilon_{V}$. Moreover,

1. the channel $\mathbb{C}_{g, \nu_{g}, \nu_{g}^{h}}$ is connected to the channel $\mathbb{C}_{g^{\prime}, \nu_{g^{\prime}}, \nu_{g^{\prime}}^{h}}$ by circles $\Gamma_{\lambda}$ of cohomology equivalence;

2. For each $c \in \operatorname{int} \mathbb{C}_{g, \nu_{g}, \nu_{g}^{h}}\left(\operatorname{int} \mathbb{C}_{g^{\prime}, \nu_{g^{\prime}}, \nu_{g^{\prime}}^{h}}\right)$ the Aubry set is located in a normally hyperbolic invariant cylinder.

By the theorem, we obtains a candidate of transition chain that connects any class $c \in \mathbb{C}_{g, \nu_{g}, \nu_{g}^{h}}$ to another class $c^{\prime} \in \mathbb{C}_{g^{\prime}, \nu_{g^{\prime}}, \nu_{g^{\prime}}^{h}}$. To make sure the candidate is indeed a transition chain we need to verify the condition (HA) for those cohomology classes in the channels for which the time-0-section of the Aubry set is an invariant circle.

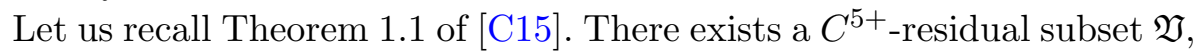
for each $V \in \mathfrak{V}$ and each irreducible class $g \in H_{1}\left(\mathbb{T}^{2}, \mathbb{Z}\right)$, there exists $\epsilon_{V}>0$ such that $\forall \epsilon \in\left[0, \epsilon_{V}\right]$ and $\forall c \in \mathbb{C}_{g, \nu_{g}, \nu_{g}^{h}}$ the time-0-section of the Mather set $\tilde{\mathcal{M}}_{0}(c)$ lies on some normally hyperbolic invariant cylinder. The number of the cylinders is independent of $\epsilon$. Indeed, $\exists$ some large $E_{i_{0}}$ such that all Mather sets $\left\{\tilde{\mathcal{M}}_{0}(c)\right\}$ lie on one cylinder if $c \in \mathbb{C}_{g, \nu_{g}, \nu_{g}^{h}}$ with $\alpha_{\epsilon}(c) \geq E_{i_{0}}$. Let $\left\{E_{i}: i=0,1, \cdots i_{0}\right\}$ be the bifurcation points, i.e. for $c \in \mathbb{C}_{g, \nu_{g}, \nu_{g}^{h}}$ with $\alpha_{0}(c)=E_{i}$, the set $\tilde{\mathcal{M}}(c)$ of $L_{0}$ is composed by two periodic orbits, while for $c$ with $\alpha_{0}(c) \neq E_{i}$, it consists of one periodic orbit (see Theorem 2.2, proved in [CZ2]).

To illustrate the result, let us work in the space $T^{*} \mathbb{T}^{2} \times \mathbb{T}$. The counterpart of $\tilde{\mathcal{M}}(c), \tilde{\mathcal{A}}(c)$ and $\tilde{\mathcal{N}}(c)$ in the space are still called Mather, Aubry and Mañé set respectively. 
Denote by $\Pi_{g, E_{i-1}, E_{i}}$ the cylinder composed of the Mather sets which are periodic orbits $\left\{x_{E}(t), y_{E}(t)\right\}$ of the Hamiltonian flow $\Phi_{G}^{t}$

$$
\Pi_{g, E_{i-1}, E_{i}}=\left\{\left(x_{E}(t), y_{E}(t)\right):\left[x_{E}\right]=g, E \in\left[E_{i-1}, E_{i}\right], t \in \mathbb{R}\right\} .
$$

As $E_{i}$ is a bifurcation point, the Mather set consists of two periodic orbits in $G^{-1}\left(E_{i}\right)$, one makes up the upper boundary of $\Pi_{g, E_{i-1}, E_{i}}$, denoted by $z_{E_{i}}^{+}$, another one makes up the lower boundary of $\Pi_{g, E_{i}, E_{i+1}}$, denoted by $z_{E_{i}}^{-}$. Due to the hyperbolicity of the orbits at both ends, the cylinder has its continuous extension $\Pi_{g, E_{i-1}-\delta, E_{i}+\delta}$ composed of the hyperbolic periodic orbits such that $G\left(x_{E}(t), y_{E}(t)\right) \in\left[E_{i-1}-\delta, E_{i}+\delta\right]$. Denoted by $x_{E}^{+}(t), y_{E}^{+}(t)$ the periodic orbit lying in $\Pi_{g, E_{i-1}-\delta, E_{i}+\delta}$ such that $G\left(x_{E}^{+}(t), y_{E}^{+}(t)\right) \in\left[E_{i}, E_{i}+\right.$ $\delta]$, by $\left(x_{E}^{-}(t), y_{E}^{-}(t)\right)$ the periodic orbit lying in $\Pi_{g, E_{i}-\delta, E_{i+1}+\delta}$ such that $G\left(x_{E}^{-}(t), y_{E}^{-}(t)\right) \in\left[E_{i}-\delta, E_{i}\right]$. It is clearly $C^{5+}$-generic condition that

(H3): $\frac{\partial G}{\partial E}\left(x_{E_{i}}^{+}(0), E_{i}\right)>\frac{\partial G}{\partial E}\left(x_{E_{i}}^{-}(0), E_{i}\right)$.

Let $G_{\epsilon}$ be the Hamiltonian obtained from $L_{\epsilon}, \Phi_{\epsilon}^{t}$ be the Hamiltonian flow of $G_{\epsilon}, \Phi_{\epsilon}=\Phi_{\epsilon}^{T}$ with $T=\left[\frac{\varrho}{\sqrt{\epsilon}}\right] \frac{\sqrt{\epsilon}}{\varrho}$. As $G_{\epsilon}$ is a perturbation of $G$, the main body of $\Pi_{g, E_{i-1}-\delta, E_{i}+\delta}$ survives perturbation $\Phi_{0} \rightarrow \Phi_{\epsilon}$, denoted by $\Pi_{g, E_{i-1}-\delta_{\epsilon}, E_{i}+\delta_{\epsilon}}^{\epsilon}$ where $\delta_{\epsilon} \uparrow \delta$ when $\epsilon \rightarrow 0$.

A point $(x, y) \in \Pi_{g, E_{i-1}-\delta_{\epsilon}, E_{i}+\delta_{\epsilon}}^{\epsilon} \operatorname{implies} G_{\epsilon}(x, y, 0) \in\left[E_{i-1}-\delta_{\epsilon}, E_{i}+\delta_{\epsilon}\right]$ as well as $\Phi_{\epsilon}(x, y) \in \Pi_{g, E_{i-1}-\delta_{\epsilon}, E_{i}+\delta_{\epsilon}}^{\epsilon}$ unless $G_{\epsilon}\left(\Phi_{\epsilon}(x, y), 0\right) \notin\left[E_{i-1}-\delta_{\epsilon}, E_{i}+\delta_{\epsilon}\right]$.

Because of the hypothesis (H3), some $E_{i, \epsilon} \rightarrow E_{i}$ exists such that the Aubry set $\tilde{\mathcal{A}}(c)$ consists of two classes if $c \in \mathbb{C}_{g, \nu_{g}, \nu_{g}^{h}}$ and $\alpha_{\epsilon}(c)=E_{i, \epsilon}$, one lies in $\Pi_{g, E_{i-1}-\delta_{\epsilon}, E_{i}+\delta_{\epsilon}}^{\epsilon}$ and the other one lies in $\prod_{g, E_{i}-\delta_{\epsilon}, E_{i+1}+\delta_{\epsilon}}^{\epsilon}$. For each $c \in \mathbb{C}_{g, \nu_{g}, \nu_{g}^{h}}$ with $\alpha_{\epsilon}(c) \in\left(E_{i-1, \epsilon}, E_{i, \epsilon}\right)$ the Aubry set lies in $\prod_{g, E_{i-1}-\delta_{\epsilon}, E_{i}+\delta_{\epsilon}}^{\epsilon}$, guaranteed by Proposition 5.2 of [C15].

Let us study the genericity of the transition chain $\Gamma_{i} \rightarrow \mathbb{C}_{g, \nu_{g}, \nu_{g}^{h}}$ so that $\alpha_{\epsilon}\left(\Gamma_{i}(0)\right)=E_{i-1, \epsilon}$ and $\alpha_{\epsilon}\left(\Gamma_{i}(1)\right)=E_{i, \epsilon}$.

Lemma 4.1. For $C^{5+}{ }_{-}$generic $V$ and $C^{4+}{ }_{\text {-generic }} R_{\epsilon}$ in Lagrangian $L_{\epsilon}$, the condition (HA) holds for each invariant circle in $\Pi_{g, E_{i-1}-\delta_{\epsilon}, E_{i}+\delta_{\epsilon}}$ with $i=1,2, \cdots i_{0}$.

Proof. The cylinder $\Pi_{g, E_{i-1}-\delta_{\epsilon}, E_{i}+\delta_{\epsilon}}^{\epsilon}$ can be thought as the image of a standard cylinder $\Pi=\left\{(x, y)^{\prime}:\left(x_{2}, y_{2}\right)^{\prime}=0, x_{1} \in \mathbb{T}, y_{1} \in[0,1]\right\}$ under the map $\psi: \Pi \rightarrow \Pi_{g, E_{i-1}-\delta_{\epsilon}, E_{i}+\delta_{\epsilon}}^{\epsilon}$. This map induces a 2 -form $\psi^{*} \omega$ on $\Pi$

$$
\psi^{*} \omega=D \psi d x_{1} \wedge d y_{1}
$$

where $D \psi$ is the Jacobian of $\psi$. Since the second de Rham cohomology group of $\Pi$ is trivial, it follows from Moser's argument on the isotopy of symplectic 
forms $[\mathrm{Mo}]$ that there exists a diffeomorphism $\psi_{1}$ on $\Pi$ such that

$$
\left(\psi \circ \psi_{1}\right)^{*} \omega=d x_{1} \wedge d y_{1} .
$$

As $\prod_{g, E_{i-1}-\delta_{\epsilon}, E_{i}+\delta_{\epsilon}}^{\epsilon}$ is invariant for the time-periodic map $\Phi_{G_{\epsilon}}$ and $\Phi_{G_{\epsilon}}^{*} \omega=\omega$, one has

$$
\left(\left(\psi \circ \psi_{1}\right)^{-1} \circ \Phi_{G_{\epsilon}} \circ\left(\psi \circ \psi_{1}\right)\right)^{*} d x_{1} \wedge d y_{1}=d x_{1} \wedge d y_{1}
$$

namely, $\left(\psi \circ \psi_{1}\right)^{-1} \circ \Phi_{G_{\epsilon}} \circ\left(\psi \circ \psi_{1}\right)$ preserves the standard area. Each invariant circle $\Gamma_{\sigma} \subset \Pi_{g, E_{i-1}-\delta_{\epsilon}, E_{i}+\delta_{\epsilon}}^{\epsilon}$ is pulled back to the standard cylinder, denoted by $\Gamma_{\sigma}^{*}$ which is Lipschitz. The parameter $\sigma$ is set to be the algebraic area bounded by the circle and a prescribed one, $\left|\Gamma_{\sigma}^{*}-\Gamma_{\sigma^{\prime}}^{*}\right|_{C^{0}} \leq C^{*} \sqrt{\left|\sigma-\sigma^{\prime}\right|}$ (see [CY1]). As the maps $\psi, \psi_{0}$ are smooth, back to the current coordinate one has $\left|\Gamma_{\sigma}-\Gamma_{\sigma^{\prime}}\right|_{C^{0}} \leq C_{1} \sqrt{\left|\sigma-\sigma^{\prime}\right|}$. We notice that the cylinder $\Pi_{g, E_{i-1}-\delta_{\epsilon}, E_{i}+\delta_{\epsilon}}^{\epsilon}$ may be crumple and slanted, the constant $C_{1}$ might approach infinity if the crumpled cylinder extends to the homoclinic orbits. However, since we keep ourselves away from the double resonance for certain distance, $E_{0}>0$ is independent of $\epsilon$, the cylinders are moderately crumpled. The constant $C_{1}$ is therefore uniformly bounded for $\sigma$ if we are restricted on the cylinder $\Pi_{g, E_{i-1}-\delta_{\epsilon}, E_{i}+\delta_{\epsilon}}^{\epsilon}$.

It is a typical phenomenon that the invariant circles make up a Cantor set. Thus, the parameter $\sigma$ is defined on a bounder Cantor set $\Sigma_{i} \subset \mathbb{R}$. If the topology on $\Sigma_{i}$ is inherited from the Euclidean metric on $\mathbb{R}$, a continuous function $c: \Sigma_{i} \rightarrow \mathbb{C}_{g, \nu_{q}, \nu_{a}^{h}}$ is obtained such that $\Gamma_{\sigma}=\tilde{\mathcal{A}}(c(\sigma))$. As the channel $\mathbb{C}_{g, \nu_{g}, \nu_{g}^{h}}$ admits a foliation of intervals $\left\{I_{\nu, g}\right\}$, each $\sigma$ corresponds to an interval. To make it single-valued function, we choose a smooth curve lying in $\mathbb{C}_{g, \nu_{g}, \nu_{g}^{h}}$ which intersects every interval $I_{\nu, g}$ transversally. Let $c=c(\sigma)$ be the intersection point of the curve with the interval corresponding to $\sigma$.

Let $\check{\pi}: \check{M} \rightarrow \mathbb{T}^{2}$ be the covering space so that the lift of $\Pi_{g, E_{i-1}-\delta_{\epsilon}, E_{i}+\delta_{\epsilon}}^{\epsilon}$ has two components, denoted by $\Pi_{i, l}$ and $\Pi_{i, u}$, where the subscript $l, u$ are introduced to indicate lower and upper respectively. In suitable coordinates one has $g=(1,0)$ and then $\check{M}=\mathbb{T} \times 2 \mathbb{T}$, namely, $x_{1} \bmod 1$ and $x_{2} \bmod 2$.

The lift of the invariant circle also has two components $\Gamma_{\sigma, l}$ and $\Gamma_{\sigma, u}$. To get a special weak KAM solution $u_{\sigma, l}^{ \pm}$, we perturb the Lagrangian $L_{\epsilon}(\dot{x}, x) \rightarrow$ $L_{\epsilon}(\dot{x}, x)+\delta V_{\delta}(x)$ with $V_{\delta} \geq 0$ and $\operatorname{supp} V_{\delta}=\pi \Gamma_{\sigma, u}+d$, where $\pi: T \check{M} \rightarrow \mathbb{T}^{2}$ is the standard projection along the tangent fiber. For $\delta>0$, a unique weak KAM solution $u_{\sigma, l, \delta}^{ \pm}$exists for $c=c(\sigma)$ such that for almost $x \in$ $\check{M} \backslash\left(\pi \Gamma_{r, \sigma}+d\right)$, the initial condition $(x, y)$ determines a unique forward (backward) semi-static orbit which approaches the circle $\Gamma_{\sigma, l}$ as $k \rightarrow \infty$ 
$(k \rightarrow-\infty)$. Let $\delta \downarrow 0$, some function $u_{\sigma, l}^{ \pm}$exists such that $u_{\sigma, l, \delta}^{ \pm} \rightarrow u_{\sigma, l}^{ \pm}$in $C^{0}$-topology. Similarly, we get another function $u_{\sigma, u}^{ \pm}$. Both $u_{\sigma, l}^{ \pm}$and $u_{\sigma, u}^{ \pm}$are weak KAM solutions and one has

$$
\begin{aligned}
& h_{c(\sigma)}^{\infty}\left(x, x^{\prime}\right)=u_{\sigma, u}^{+}\left(x^{\prime}, 0\right)-u_{\sigma, u}^{+}(x, 0), \quad x^{\prime} \in \pi \Gamma_{\sigma, u} \\
& h_{c(\sigma)}^{\infty}\left(x^{\prime}, x\right)=u_{\sigma, l}^{-}(x, 0)-u_{\sigma, l}^{-}\left(x^{\prime}, 0\right), \quad x^{\prime} \in \pi \Gamma_{\sigma, l} .
\end{aligned}
$$

The normal hyperbolicity guarantees the smooth dependence of stable (unstable) fibers on the base points, the local stable (unstable) manifolds of $\Gamma_{\sigma, l}, \Gamma_{\sigma, u}$ are also parameterized by $\sigma$ with $\frac{1}{2}$-Hölder continuity. They are the time-0-section of the graph of $\partial u_{\sigma, l}^{ \pm}$and $\partial u_{\sigma, u}^{ \pm}$respectively. So, $\left|u_{\sigma, l}^{ \pm}-u_{\sigma^{\prime}, l}^{ \pm}\right|_{C^{0}} \leq C_{1} \sqrt{\left|\sigma-\sigma^{\prime}\right|}$ holds in a neighborhood $\pi \Gamma_{\sigma, \imath}+d$ of the circle $\Gamma_{\sigma, \imath}$ for $\imath=l, u$.

In the region $\check{M} \backslash\left(\pi \Gamma_{\sigma, l}+\delta\right) \cup\left(\pi \Gamma_{\sigma, u}+\delta\right)$ with $0<\delta<d$, the stable and unstable manifold may not keep horizontal any more, because of conjugate point. As a result, it seems not so clear that the Hölder modulus continuity hold in this region. However, due to Lemma 6.4 in [CY2] (substitute $h_{c}^{\infty}$ with the formulae (4.2)), one has

$$
\begin{aligned}
& \max _{x \in \bar{M} \backslash \pi \Gamma_{\sigma, u}+\delta}\left|u_{\sigma, l}^{ \pm}(x, 0)-u_{\sigma^{\prime}, l}^{ \pm}(x, 0)\right| \leq C_{2}\left(\left|\sigma-\sigma^{\prime}\right|^{\frac{1}{2}}+\left|c(\sigma)-c\left(\sigma^{\prime}\right)\right|\right), \\
& \max _{x \in \bar{M} \backslash \pi \Gamma_{\sigma, l}+\delta}\left|u_{\sigma, u}^{ \pm}(x, 0)-u_{\sigma^{\prime}, u}^{ \pm}(x, 0)\right| \leq C_{2}\left(\left|\sigma-\sigma^{\prime}\right|^{\frac{1}{2}}+\left|c(\sigma)-c\left(\sigma^{\prime}\right)\right|\right) .
\end{aligned}
$$

The rest of the proof is completed by applying the argument in Section 6 of [CY1]. Under the perturbation of bump function as designed there, the set of barrier function undergoes a translation $\left\{B_{c(\sigma)}^{*}\right\} \rightarrow\left\{B_{c(\sigma)}^{*}+\delta G\right\}$ when they are restricted on a disk $D$ where the minimal homoclinic curves pass through. We define a set $\mathfrak{Z} \subset C^{0}$. A function $U \in \mathfrak{Z}$ if the set $U^{-1}(\min U) \cap D$ is not totally disconnected. This set has infinite "codimensions" in $C^{0}\left(\mathbb{T}^{3}\right)$. Since the box dimension of $\left\{B_{c(\sigma)}^{*}\right\}$ is finite, there are abundant perturbations of shift $\left\{B_{c(\sigma)}^{*}\right\} \rightarrow\left\{B_{c(\sigma)}^{*}+\delta G\right\}$ so that $\left\{B_{c(\sigma)}^{*}+\delta G\right\} \cap \mathfrak{Z}=\varnothing$. It implies that all minimal homoclinic orbits are totally disconnected for the perturbed Lagrangian. Clearly, the perturbation can be arbitrarily small. For the details, one can refer to Section 6 of [CY1].

To study the (HA) condition for invariant circles in high energy level set, in virtue of the result in [C15], we only need to consider one cylinder which extends from certain energy level with large $E$ but independent of $\epsilon$ to the level where $E=O\left(\epsilon^{2 \kappa-1}\right)$.

To illustrate why there does not exist bifurcation point so that one cylinder extends for such a large scale, we notice that along any periodic orbit 
$(x(t), y(t))$ of $\Phi_{G}^{t}$ lying in high energy level set the quantity $|y(t)|$ is large. For $g=(1,0)$ and large $\nu \in \mathbb{Z}$, in the new coordinates $y \rightarrow y-y^{\prime}, x=x$ where $y^{\prime}=\nu A g$ the Hamiltonian $G$ appears to be (up to a constant)

$$
G=\nu y_{1}+\frac{1}{2}\left\langle A^{-1} y, y\right\rangle-V(x) .
$$

under further coordinate rescaling $\left(x_{1}, x_{2}, y_{1}, y_{2}\right) \rightarrow\left(\frac{x_{1}}{\nu}, x_{2}, \nu y_{1}, y_{2}\right)$ one obtains a time-periodic Hamiltonian with one degrees of freedom

$$
y_{1}=\frac{1}{2} a y_{2}^{2}+b E y_{2}-V\left(\nu x_{1}, x_{2}\right)+\frac{1}{\nu} R_{H}\left(\nu x_{1}, x_{2}, y_{2}, E\right)+\text { const. }
$$

which solves the equation $G\left(\frac{x_{1}}{\nu}, x_{2},-\nu y_{1}\left(\nu x_{1}, x_{2}, y_{2}\right), y_{2}\right)=\nu E, a$ and $b$ are constants with $a>0$ the remainder $\frac{1}{\nu} R_{H}$ is of order $\frac{1}{\nu}$ in $C^{r}$-topology in the variable $\left(\nu x_{1}, x_{2}, y_{2}\right)$. One gains a Lagrangian with one and half degrees of freedom from this Hamiltonian

$$
L^{\prime}=\frac{1}{2 a} \dot{x}_{2}^{2}+\frac{b E}{a} \dot{x}_{2}+V\left(\nu x_{1}, x_{2}\right)+\frac{1}{\nu} R_{L}\left(\nu x_{1}, x_{2}, \dot{x}_{2}, E\right) .
$$

The problem to find minimal periodic orbits of $L_{0}$ with the class $g$ and large energy is switched to the problem to find the minimizer of the function

$$
F\left(x_{2}, \nu, E\right)=\inf _{\gamma(0)=\gamma(1)=x_{2}} \int_{0}^{1} L^{\prime}(\nu \tau, \gamma(\tau), \dot{\gamma}(\tau)) d \tau
$$

The term $\frac{b E}{a} \dot{x}_{2}$ is a closed 1-form, does not contribute the function $F\left(x_{2}, E\right)$. Applying the technique to prove Riemann-Lebesgue lemma one obtains a decomposition of the function $F=F_{0}+\frac{1}{\mu} F_{R}$ where

$$
F_{0}\left(x_{2}, \nu, E\right)=\int_{0}^{2 \pi}\left(\frac{1}{2 a}\left(\dot{\gamma}_{\nu, E}\left(\tau, x_{2}\right)\right)^{2}+[V]\left(\gamma_{\nu, E}\left(\tau, x_{2}\right)\right)\right) d \tau,
$$

where $[V]=\int_{0}^{1} V\left(\nu \tau, x_{2}\right) d \tau, \gamma_{\nu, E}$ is the minimal curve of $F\left(x_{2}, \nu, E\right)$. Thus, the minimal curve is $C^{1}$-close to a straight line $\gamma(\tau)=x_{2} \in[V]^{-1}(\min [V])$. For generic $V$, the minimal point of $[V]$ is unique and non-degenerate. As the term $\frac{1}{2 a} \dot{x}_{2}^{2}+[V]\left(x_{2}\right)$ is independent of $\nu$ and $E$, such a cylinder exists and extends from certain energy level with large $E$ but independent of $\epsilon$ to the level set where $E=O\left(\epsilon^{2 \kappa-1}\right)$. One can refer to Section 3 of [C15] for the details.

Lemma 4.2. For $C^{5+}$-generic $V$ and $C^{4+}$-generic $R_{\epsilon}$ in Lagrangian $L_{\epsilon}$, the condition (HA) holds for each invariant circle in $\Pi_{g, E_{i_{0}}-\delta_{\epsilon}, \epsilon^{2 \kappa-1}}$. 
Proof. To check the condition (HA) for the cylinder $\Pi_{g, E_{i_{0}}-\delta_{\epsilon}, \epsilon^{2 \kappa-1}}^{\epsilon}$, we recall that the cylinder $\Pi_{g, E_{i_{0}}, \epsilon^{2 \kappa-1}}$ is composed by minimal periodic orbits of $G$ with high energy if $E_{i_{0}}>0$ is large. By Proposition 3.1 in [C15], the minimal periodic orbit in $G^{-1}(E)$ remains in a small neighborhood of the circle $\left\{(x, y):\left(x_{2}, y\right)=\right.$ const. $\}$ if $E>0$ is suitably large. Therefore, the cylinder $\Pi_{g, E_{i_{0}}-\delta_{\epsilon}, \epsilon^{2 \kappa-1}}^{\epsilon}$ is a slight deformation of standard cylinder, the map $\Phi_{\epsilon}$ is close to integrable when it is restricted on the cylinder. Thus, the constant $C_{1}$ in the parameterization so that $\left|\Gamma_{\sigma}-\Gamma_{\sigma^{\prime}}\right|_{C^{0}} \leq C_{1} \sqrt{\left|\sigma-\sigma^{\prime}\right|}$ is uniformly bounded for the whole cylinder. Although the quantity

$$
\max \left\{\left|\sigma-\sigma^{\prime}\right|: \Gamma_{\sigma}, \Gamma_{\sigma^{\prime}} \subset \Pi_{g, E_{i_{0}}-\delta_{\epsilon}, \epsilon^{2 \sigma-1}}^{\epsilon}\right\}
$$

approaches infinity as $\epsilon \rightarrow 0$, it is finite once $\epsilon>0$ is fixed. As we only need to show the density of (HA) i.e. for each $\sqrt{\epsilon} R_{\epsilon}$ some other $\sqrt{\epsilon} R_{\epsilon}^{\prime}$ exists such that $\left|\sqrt{\epsilon} R_{\epsilon}^{\prime}-\sqrt{\epsilon} R_{\epsilon}\right|$ can be arbitrarily small and (HA) holds for $\sqrt{\epsilon} R_{\epsilon}^{\prime}$. It implies the number $\epsilon>0$ is fixed when we handle this problem. So, the parameter $\sigma$ is restricted in a bounded set, and consequently, the argument for $\Pi_{g, E_{i-1}-\delta_{\epsilon}, E_{i}+\delta_{\epsilon}}^{\epsilon}$ applies here.

With Lemma 4.1, Lemma 4.2 and the annulus of cohomology equivalence we see that a generalized transition chain has been established to join a class $c \in \mathbb{C}_{g, \nu_{g}, \nu_{g}^{h}}$ and another class $c^{\prime} \in \mathbb{C}_{g^{\prime}, \nu_{g}^{\prime}, \nu_{g}^{\prime h}}$. It passes through the channel $\mathbb{C}_{g, \nu_{g}, \nu_{g}^{h}}$ down to lower energy level set, then turns around the double resonance along circles of cohomology equivalence before it reaches the channel $\mathbb{C}_{g^{\prime}, \nu_{g}^{\prime}, \nu_{g}^{\prime h}}$, through which it arrives at $c^{\prime}$.

As a matter of fact, the channel can be deeply extended to lower energy level set $\alpha_{\epsilon}^{-1}\left(O\left(\epsilon^{d}\right)\right)$ where $d<\vartheta \kappa$, the constant $\vartheta<\frac{1}{4}$ only depends on $L_{0}$, see Formula (2.29) in [C15].

For Lagrangian $L_{0}$ and each $\nu \in\left(0, \nu_{0}\right]$, it follows from Proposition 2.6 that the $\nu g$-minimal minimal periodic orbit lies on some NHIC. In terms of Poincaré return map, the normal hyperbolicity approaches infinity as $\nu \downarrow 0$ (see Lemma 2.3), meanwhile the return time approaches infinity also. The theorem of implicit function guarantees that certain NHIC extends from $\nu=+0$ to $\nu=\nu_{0}$. Thus, there are finitely many cylinders only, since hyperbolic fixed points are isolated. Denote by $\Pi_{g, 0, E_{0}}^{\ell}$ the cylinder where $E_{0}=\alpha_{0}\left(\mathscr{L}_{\beta_{0}}\left(\nu_{0} g\right)\right), \ell=1,2, \cdots, \ell_{0}$. One does not expect the whole cylinder survives the time-periodic perturbation $\sqrt{\epsilon} R_{\epsilon}$, but large part of $\Pi_{g, 0, \nu_{0}}^{\ell}$ survives. An invariant manifold with boundary is said to be overflowing if any point leaves or enters the manifold only by passing through its boundary.

Lemma 4.3. Let $\Phi_{\epsilon}^{t}$ be the Hamiltonian flow of $G_{\epsilon}$ obtained from the Lagrangian $L_{\epsilon}$ by the Legendre transformation, then 
1. The Hamiltonian flow $\Phi_{\epsilon}^{t}$ admits an overflowing invariant cylinder $\tilde{\Pi}_{g, \epsilon^{d}, E_{0}+\delta_{\epsilon}}^{\epsilon}$ in $T^{*} \mathbb{T}^{2} \times \varrho \sqrt{\epsilon} \mathbb{T}\left(\varrho=1, \frac{1}{\sqrt{\epsilon}}\right)$ which lies in an $\epsilon^{\kappa}$-neighborhood of $\Pi_{g, \epsilon^{d}, E_{0}+\delta_{\epsilon}} \times \varrho \sqrt{\epsilon} \mathbb{T}$, the point $(x, y, t) \in \tilde{\Pi}_{g, \epsilon^{d}, E_{0}+\delta_{\epsilon}}^{\epsilon, \ell}$ if $\frac{1}{2} \epsilon^{d} \leq G(x, y) \leq$ $E_{0}+\delta_{\epsilon}-\epsilon^{d}$;

2. There is a number $N>1$ such that for a class $c \in \mathbb{C}_{g}$ with $\alpha_{\epsilon}(c) \geq$ $N \epsilon^{d}$, the Aubry set $\tilde{\mathcal{A}}(c)$ lies in the set $\tilde{\Pi}_{g, \epsilon^{d}, E_{0}+\delta_{\epsilon}}^{\epsilon,}$ Indeed, each orbit in the Aubry set does not hit the energy level set $G_{\epsilon}^{-1}(E)$ with $E \leq \epsilon^{d}$.

Proof. The condition is not required that $\Pi_{g, 0, E_{0}}^{\ell}$ is completely foliated by $\nu g$-minimal periodic orbits of $L_{0}$. If the Aubry set $\tilde{\mathcal{A}}(c)$ is contained in $\tilde{\Pi}_{g, \epsilon^{d}, E_{0}+\delta_{\epsilon}}^{\epsilon, \ell}$ by perturbing the Lagrangian $L_{0} \rightarrow L_{0}^{\prime}$ to increase the action along curves on other cylinders, one can assume the condition holds for $L_{0}^{\prime}$ while $L_{0}^{\prime}=L_{0}$ when they are restricted in a neighborhood of $\tilde{\Pi}_{g, \epsilon^{d}, E_{0}+\delta_{\epsilon}}$. Therefore, one has $L_{0}^{\prime}+\sqrt{\epsilon} R_{\epsilon}=L_{\epsilon}$ in the neighborhood.

Since the Hamiltonian $G_{\epsilon}$ is obtained from $L_{\epsilon}$ by the Legendre transformation, it is a time-periodic $\epsilon^{\kappa}$-perturbation of $G$, namely, $G_{\epsilon}=G+\epsilon^{\kappa} R_{\epsilon}$.

To modify the Hamiltonian $G_{\epsilon}$ we choose a $C^{2}$-function $\rho$ so that $\rho(\nu)=$ 1 for $\nu \geq 1, \rho(\nu)=0$ for $\nu \leq 0$ and set $\rho_{2}=1-\rho\left(\frac{G(x, y)-\left(E_{0}+\delta_{\epsilon}-\epsilon^{d}\right)}{\epsilon^{d}}\right)$, $\rho_{1}(x, y)=\rho\left(2 \frac{G(x, y)-\epsilon^{3 d}}{\epsilon^{d}-2 \epsilon^{3 d}}\right)$. We set

$$
G_{\epsilon}^{\prime}= \begin{cases}G+\epsilon^{\sigma} \rho_{1} R_{\epsilon}, & \text { if } G(x, y) \in\left[\epsilon^{3 d}, \frac{1}{2} \epsilon^{d}\right], \\ G+\epsilon^{\sigma} \rho_{2} R_{\epsilon}, & \text { if } G(x, y) \in\left[E_{0}+\delta_{\epsilon}-\epsilon^{d}, E_{0}+\delta_{\epsilon}\right], \\ G_{\epsilon}, & \text { elsewhere. }\end{cases}
$$

Clearly, $\left\|G_{\epsilon}^{\prime}-G\right\|_{C^{2}} \ll 1$ if $d<\kappa$ and $\epsilon \ll 1$. It follows that the cylinder $\Pi_{g, \epsilon^{3 d}, E_{0}+\delta_{\epsilon}}$ persists the perturbation $\Phi_{G} \rightarrow \Phi_{G_{\epsilon}^{\prime}}$, the boundary of the cylinder remains unchanged. We use the notation $\Phi_{G_{\epsilon}^{\prime}}=\left.\Phi_{G_{\epsilon}^{\prime}}^{t, t+1}\right|_{t=0}$ where $\Phi_{G_{\epsilon}^{\prime}}^{t, t^{\prime}}(x, y)$ denotes a point on the orbit of the Hamiltonian flow that emanate from the point $(x, y)$ at the time $t$ and arrive at this point after the time $t^{\prime}-t$. The survived cylinder in the phase space $\mathbb{T}^{2} \times \mathbb{R}^{2} \times \mathbb{T}$ is denoted by $\tilde{\Pi}_{g, \epsilon^{3 d}, E_{0}+\delta_{\epsilon}}^{\epsilon}$. Since $G_{\epsilon}=G_{\epsilon}^{\prime}$ when they are restricted to $\tilde{\Pi}_{g, \epsilon^{3 d}, E_{0}+\delta_{\epsilon}}^{\epsilon} \cap$ $\left\{(x, y, t): G \in\left[\frac{1}{2} \epsilon^{d}, E_{0}+\delta_{\epsilon}-\epsilon^{d}\right]\right\}$, it completes the proof for the first part.

For the second part, we only need to prove it for the Hamiltonian $G_{\epsilon}^{\prime}$ of (4.4). So, each orbit in the Aubry set lies in the cylinder forever. If the lemma does not hold, there would be an orbit $z(t)=(x(t), y(t))$ lying in the Aubry set for $c \in \mathbb{C}_{g} \cap \alpha_{\epsilon}^{-1}\left(N \epsilon^{d}\right)$ hitting the energy level $G_{\epsilon}^{-1}\left(\epsilon^{d}\right)$ at the time $t_{0}$, namely, $G_{\epsilon}\left(z\left(t_{0}\right), t_{0}\right)=\epsilon^{d}$. It returns to a neighborhood of $z\left(t_{0}\right)$ 
after a time $t^{\prime}=O\left(\left|\ln \epsilon^{d}\right|\right)($ cf. Formula $(2.22)$ of $[\mathrm{C} 15])$. Because $\frac{d G}{d t}=\frac{\partial G}{\partial t}$, for time-1-periodic case, one has

$$
\left|G_{\epsilon}^{\prime}\left(z\left(t^{\prime}+t_{0}\right), t^{\prime}+t_{0}\right)-G_{\epsilon}^{\prime}\left(z\left(t_{0}\right), t_{0}\right)\right| \leq K t^{\prime} \epsilon^{\kappa} .
$$

For time- $\frac{1}{\varrho \sqrt{\epsilon}}$-periodic case, along an orbit $z(s)$ of the Hamiltonian flow, the variation of the energy is controlled by

$$
\frac{d}{d t} G_{\epsilon}(z(t), t)=\frac{\partial}{\partial t} G_{\epsilon}(z(t), t)=\epsilon^{\kappa-\frac{1}{2}} \frac{\partial R_{\epsilon}}{\partial \theta}
$$

Since $R_{\epsilon}$ is 1-periodic in $\frac{1}{\varrho \sqrt{\epsilon}}$, we expend $R_{\epsilon}$ into Fourier series of $\frac{t}{\varrho \sqrt{\epsilon}}$

$$
\frac{\partial G_{\epsilon}(z, t)}{\partial t}=\epsilon^{\kappa-\frac{1}{2}} \sum_{\ell \neq 0} \frac{i \ell}{\varrho} R_{\epsilon, \ell}(z) e^{i \ell \frac{t}{\varrho \sqrt{\epsilon}}}
$$

Integrating by parts, we have

$$
\begin{aligned}
\frac{i \ell}{\varrho} \epsilon^{\kappa-\frac{1}{2}} \int_{t_{0}}^{t_{1}} R_{\epsilon, \ell}(z(t)) e^{i \ell \frac{t}{\varrho \sqrt{\epsilon}}} d t & =\left.\epsilon^{\kappa} R_{\epsilon, \ell}(z(t)) e^{i \ell \frac{t}{\varrho \sqrt{\epsilon}}}\right|_{t_{0}} ^{t_{1}} \\
& -\epsilon^{\kappa} \int_{t_{0}}^{t_{1}}\left\langle\partial R_{\epsilon, \ell}, \dot{z}(t)\right\rangle e^{i \ell \frac{t}{\varrho \sqrt{\epsilon}}} d t .
\end{aligned}
$$

Because the perturbation term $R_{\epsilon}$ is $C^{4}$-smooth, one has $\left|\partial_{z} R_{\epsilon, \ell}\right| \leq \frac{\left\|\partial_{z} R_{\epsilon}\right\|_{C^{3}}}{2 \pi|\ell|^{3}}$. Since $z(t)$ solves the equation $\dot{z}(t)=J \nabla_{z} G_{\epsilon}(z(t), t)$, by setting

$$
K=\max _{(z, t) \in \tilde{\Pi}}\left\|\nabla_{z} G_{\epsilon}(z(t), t)\right\|\left\|\partial_{z} R_{\epsilon}\right\|_{C^{3}} \sum_{\ell \neq 0} \frac{1}{|\ell|^{4}},
$$

where the maximum is taken over the cylinder $\tilde{\Pi}_{g, \epsilon^{d}, E_{0}}^{\epsilon}, K$ is uniformly upper bounded for small $\epsilon$. It follows from (4.6) and (4.7) that

$$
\begin{aligned}
& \left|G_{\epsilon}\left(z\left(t_{1}\right), t_{1}\right)-G_{\epsilon}\left(z\left(t_{0}\right), t_{0}\right)\right|=\left|\int_{t_{0}}^{t_{1}} \frac{\partial}{\partial t} G_{\epsilon}(z(t), t) d t\right| \\
& \leq\left.\epsilon^{\kappa} R_{\epsilon}(z(t), t)\right|_{t_{0}} ^{t_{1}}+\epsilon^{\kappa}\left|\sum_{\ell \neq 0} \int_{t_{0}}^{t_{1}}\left\langle\partial R_{\epsilon, \ell}, \dot{z}(t)\right\rangle e^{i \ell \frac{t}{\varrho \sqrt{\epsilon}}} d t\right|
\end{aligned}
$$

the right hand side is bounded by $K\left(t_{1}-t_{0}\right) \epsilon^{\kappa}$, namely, the estimate (4.5) holds also in time- $\frac{1}{\varrho \sqrt{\epsilon}}$-periodic case.

As $G^{-1}(E) \cap \Pi_{g, \epsilon^{d}, E_{0}}$ is an invariant circle for $\Phi_{G}$, the perturbed cylinder is $O\left(\epsilon^{\kappa}\right)$-close to the original one in $C^{r-1}$-topology. The cylinder may be 
crumpled but at most up to the order $O\left(\epsilon^{d \varsigma}\right)$ see (4.10) below, so there is a time $T=O\left(\left|\ln \epsilon^{d}\right|\right)$ and a small number $\mu^{\prime}=d \varsigma>0$ such that $\kappa-\mu^{\prime}>0$ and

$$
\left.\| z\left(T+t_{0}\right)-z\left(t_{0}\right)\right) \| \leq C T \epsilon^{\kappa-\mu^{\prime}} .
$$

By decreasing $d \rightarrow d^{\prime}$ such that $d^{\prime}-d=O\left(|\ln \epsilon|^{-1}\right)$ one can assume $T$ is an integer in time-1-periodic case. In time- $\frac{1}{\varrho \sqrt{\epsilon}}$-periodic case, $T$ is very close to $\left[\frac{T}{\varrho \sqrt{\epsilon}}\right] \varrho \sqrt{\epsilon}$.

Since $x(t)$ is $c$-static, $\left|x\left(t_{0}\right)-x\left(t_{0}+T\right)\right| \leq C T \epsilon^{\kappa-\mu^{\prime}}$ the following holds in both cases

$$
\left|\int_{t_{0}}^{T+t_{0}}\left(L_{\epsilon}(x(t), \dot{x}(t), t)-\langle c, \dot{x}(t)\rangle+\alpha_{\epsilon}^{\prime}(c)\right) d t\right| \leq C^{\prime} T \epsilon^{\kappa-\mu^{\prime}} .
$$

However, by applying the following observation

1. the $\alpha_{\epsilon}$-function for $G_{\epsilon}$ is a small perturbation of $\alpha_{0},\left|\alpha_{\epsilon}-\alpha_{0}\right| \leq \epsilon^{\kappa}$ (see $[\mathrm{C} 11]$ );

2. for $c \in \mathbb{C}_{g}, \alpha_{0}$ is smooth and strictly convex in the direction of $g$, its derivative in the direction of $g$ is the frequency that is of the order $O\left(|\ln \epsilon|^{-1}\right)$;

3. for $c^{\prime} \in \mathbb{C}_{g} \cap \alpha_{0}^{-1}\left(\epsilon^{d}\right)$, each $c^{\prime}$-static orbit for $G$ keeps $|\ln \epsilon| \epsilon^{\kappa-\mu^{\prime}}$-close to the segment $\left.(x, \dot{x})\right|_{\left[t_{0}, t_{0}+T\right]}$, while $\alpha_{\epsilon}(c)-\alpha_{0}\left(c^{\prime}\right) \geq(N-1) \epsilon^{d}$ and $\kappa>4 d$.

we find that the left hand side of (4.9) is in fact bounded from below by $C \epsilon^{d}$ where $C>0$ is a constant. One can refer to the proof of Proposition 5.1 of [C15] for the details. As $d<\frac{1}{4} \kappa, \mu^{\prime}$ is small and $T=O(|\ln \epsilon|)$ it leads to a contradiction for small $\epsilon$.

Let $\Pi_{g, \epsilon^{d}, E_{0}+\delta_{\epsilon}}^{\epsilon, \ell}$ be the time-0-section, a 2-dimensional cylinder. All invariant circles on the cylinder are Lipschitz and are parameterized so that $\left|\Gamma_{\sigma}-\Gamma_{\sigma^{\prime}}\right|_{C^{0}} \leq C_{1}^{\prime} \sqrt{\left|\sigma-\sigma^{\prime}\right|}$ as shown before. However, the coefficient $C_{1}^{\prime}$ may be much larger than $C^{*}$ since the cylinder may be crumpled. Since the cylinder $\Pi_{g, \epsilon^{d}, E_{0}+\delta_{\epsilon}}^{\epsilon, \ell}$ is a $C^{2}$-perturbation of $\Pi_{g, \epsilon^{d}, E_{0}+\delta_{\epsilon}}^{\ell}$ of order $O\left(\epsilon^{\kappa}\right)$, we only need to consider the unperturbed one. As the homoclinic orbits in all relevant Aubry sets are assumed to approach the origin in the direction of $\Lambda_{1}$, if the cylinder is the image of the map $\left(x_{1}, y_{1}\right) \rightarrow\left(x_{1}, x_{2}, y_{1}, y_{2}\right)$, one has

$$
\left.\omega\right|_{\prod_{g, \epsilon^{\epsilon}, E_{0}+\delta_{\epsilon}}^{\epsilon, \ell}}=\left(1+\frac{\partial\left(x_{2}, y_{2}\right)}{\partial\left(x_{1}, y_{1}\right)}\right) d x_{1} \wedge d y_{1} .
$$

Since we are concerned about the cylinders not touching the region where $G_{\epsilon}<\epsilon^{d}$, the formula $(2.22)$ of [C15] shows 


$$
\left|\frac{\partial x_{2}}{\partial y_{1}}\right|,\left|\frac{\partial y_{2}}{\partial y_{1}}\right| \leq C E^{-\varsigma}, \quad \text { for }(x, y) \in G^{-1}(E)
$$

where $\varsigma>0$ is very small. Therefore, the coefficient $C_{1}^{\prime}$ is bounded by $O\left(\epsilon^{-d \varsigma}\right)$. To get an upper bound of $C_{1}^{\prime}$ in general case, we work in the covering space $\bar{M}=\bar{k}_{1} \mathbb{T} \times \bar{k}_{2} \mathbb{T}$ where $\bar{k}_{m}=k_{i} g_{i, m}+k_{i+1} g_{i+1, m}$ for $m=1,2$ if we write $g_{\ell}=\left(g_{\ell, 1}, g_{\ell, 2}\right)$ for $\ell=i, i+1$. By a coordinate transformation on $\bar{M}$ we can still assume the cylinder is the image of the map $\left(x_{1}, y_{1}\right) \rightarrow\left(x_{1}, x_{2}, y_{1}, y_{2}\right)$. Although there are $k_{i}+k_{i+1}$ fixed points, they are the lift of the hyperbolic fixed point. The analysis for upper bound of $C_{1}^{\prime}$ is the same.

To complete the proof, we emphasize that the large Hölder coefficient $C_{1}^{\prime}=O\left(\epsilon^{-d \varsigma}\right)$ does not damage the proof, since it is only crucial to have a positive Hölder exponent uniformly bounded away from zero. Although $\epsilon$ is small, it is fixed in the context. We only need to show some other $\sqrt{\epsilon} R_{\epsilon}^{\prime}$ exists such that $\left|\sqrt{\epsilon} R_{\epsilon}-\sqrt{\epsilon} R_{\epsilon}^{\prime}\right|$ is arbitrarily small and (HA) condition holds for $\sqrt{\epsilon} R_{\epsilon}^{\prime}$. To show the transversal intersection of the stable and unstable manifolds, we parameterize the classes by $\sigma$ such that (4.3) holds. A Hamiltonian produces a set of barrier functions $H \rightarrow\left\{B_{c(\sigma)}^{*}\right\}$. As $\sigma$ is defined on a Cantor set on line, the box dimension of $\left\{B_{c(\sigma)}^{*}\right\}$ is not larger than 2 in $C^{0}$-topology, due to the Hölder exponent in (4.3) which equals $\frac{1}{2}$.

As the conclusion of the subsection, Theorem 1.2 is proved.

Remark. We do not need to consider the case that cylinder extends to the lowest energy level $\alpha_{\epsilon}^{-1}\left(\min \alpha_{\epsilon}\right)$ even if $g=g_{i}$, namely, the cylinder $\Pi_{g_{i}, 0, E_{0}}$ takes a homoclinic orbit $z_{g_{i}}$ as its boundary. The precise quantitative analysis of cylinder around the fixed point appears to be complicated, but it is not required in our approach.

\subsection{Genericity in the sense of Mañé}

The method developed in [CY1] does not apply to prove the genericity if the perturbation $\sqrt{\epsilon} R_{\epsilon}$ is independent of $\dot{x}$. In this subsection, let us prove the genericity of transition chain in the category of potential perturbation, namely, in the sense of Mañé.

To check the condition (HA) for all invariant circles in the cylinder $\Pi_{g, E_{i-1}-\delta_{\epsilon}, E_{i}+\delta_{\epsilon}}^{\epsilon}$, we can assume $g=(1,0)$ and work in the covering space $\mathbb{T} \times 2 \mathbb{T}$. Then, the time-periodic section of Aubry set consists of two invariant circles $\Gamma_{\sigma, l}$ and $\Gamma_{\sigma, u}$ for each $\sigma \in \Sigma_{i}$. The subscript $l, u$ mean lower, upper respectively.

For convenience of notation, we work in the extended space $\tilde{x}=\left(x, x_{3}\right) \in$ $\mathbb{T} \times 2 \mathbb{T} \times \mathbb{T}$ where $\dot{x}_{3}=1$, each invariant circle $\Gamma_{\sigma, \imath}$ is expanded into a 2-torus $\tilde{\Gamma}_{\sigma, \imath}$ with $\imath=l, u$. Let $\tilde{\gamma}(t)=(\gamma(t), t)$ denote curve in the extended space. 
Due to the normal hyperbolicity, the circle $\tilde{\Gamma}_{\sigma, \imath}$ has its stable, unstable manifold $\tilde{W}_{\sigma, \imath}^{ \pm}$for $\imath=l, u$. There exists a neighborhood $U_{\sigma, \imath}$ of $\pi \tilde{\Gamma}_{\sigma, \imath}$, over which $\tilde{W}_{\sigma, \imath}^{ \pm}$keeps horizontal, i.e. there is a generating function $u_{\sigma, \imath}^{ \pm}$such that $\tilde{W}_{\sigma, \imath}^{ \pm}=\operatorname{Graph} d u_{\sigma, \imath}^{ \pm}$. This function is at least $C^{1,1}$-smooth when it is restricted on $U_{\sigma, \imath}$ and has its extension to the whole space, which is a weak KAM solution of the Hamilton-Jacobi equation.

Any $c(\sigma)$-minimal curve in the Mañé set has to pass through $U_{\sigma, l} \backslash\left(\pi \tilde{\Gamma}_{\sigma, l}+\right.$ $\delta$ ) when it approaches $\pi \tilde{\Gamma}_{\sigma, l}$, the duration approaches infinity as $\delta \rightarrow 0$. Let $\mathrm{T} \subset U_{\sigma, l} \backslash\left(\pi \tilde{\Gamma}_{\sigma, l}+\delta^{\prime}\right)$ be a 2-dim torus homological to $\pi \tilde{\Gamma}_{\sigma, l}$. To check the condition (HA) we only need to investigate the set

$$
\left.\arg \min \left(u_{\sigma, l}^{-}-u_{\sigma, u}^{+}\right)\right|_{\mathrm{T}}=\left\{\tilde{x} \in \mathrm{T}:\left(u_{\sigma, l}^{-}-u_{\sigma, u}^{+}\right)(\tilde{x})=\min \left(u_{\sigma, l}^{-}-u_{\sigma, u}^{+}\right)\right\}
$$

and prove

Theorem 4.2. There exists a set $\mathfrak{V}_{\infty}$ residual in $C^{r}\left(\mathbb{T}^{2} \times \mathbb{T}, \mathbb{R}\right)$ such that, for each $V \in \mathfrak{V}_{\infty}$, it holds simultaneously for all $\sigma \in \mathbb{I}_{\sigma}$ that the set $\left.\arg \min \left(u_{\sigma, l}^{-}-u_{\sigma, u}^{+}\right)\right|_{\mathrm{T}}$ is totally disconnected.

Proof. Dividing the torus $\mathrm{T}$ into squares with the side length $d$. Pick up one of them, we denote it by $D$. Under a local coordinate transformation, a neighborhood of $D$ can be assumed to be

$$
D+d^{\prime}=\left\{\tilde{x}:\left|x_{1}-x_{1,0}\right| \leq d+d^{\prime}, x_{2}=x_{2,0},\left|x_{3}-x_{3,0}\right| \leq d+d^{\prime}\right\},
$$

and $D=D+\left.d^{\prime}\right|_{d^{\prime}=0}$. For a subset $S \subset D$, let $\Pi_{i} S$ be its project to the $x_{i^{-}}$ coordinate, $\Pi_{i} S=\left\{x_{i}: \tilde{x} \in S,\left|x_{i}-x_{i, 0}\right| \leq d\right\}$ for $i=1,3$. If for each of the squares and for $i=1,3$, one has $\left.\Pi_{i} \arg \min \left(u_{\sigma, l}^{-}-u_{\sigma, u}^{+}\right)\right|_{D} \subsetneq\left[x_{i, 0}-d, x_{i, 0}+d\right]$, the diameter of each connected component of $\left.\arg \min \left(u_{\sigma, l}^{-}-u_{\sigma, u}^{+}\right)\right|_{\mathrm{T}}$ will not be larger than $2 d$.

To construct potential perturbation, we do some preparatory work. Let $\tilde{\gamma}_{\sigma, l}^{-}, \tilde{\gamma}_{\sigma, u}^{+}$be a backward and forward $c(\sigma)$-semi static curve approaching $\pi \tilde{\Gamma}_{\sigma, l}, \pi \tilde{\Gamma}_{\sigma, u}$ as $t \rightarrow-\infty$ and $t \rightarrow \infty$ respectively. Starting from a point on $\mathrm{T}$, a unique curve $\tilde{\gamma}_{\sigma, l}^{-}$will retreat back down to the torus $\pi \tilde{\Gamma}_{\sigma, l}$ as $t \rightarrow-\infty$. We denote by $\tilde{\gamma}_{\sigma, l}^{ \pm}(t, \tilde{x})$ the curve such that $\tilde{\gamma}_{\sigma, l}^{ \pm}(0, \tilde{x})=\tilde{x}$ for $\imath=l, u$. We write the curve $\tilde{\gamma}_{\sigma, l}^{ \pm}(\cdot, \tilde{x})$ in the coordinate form when it is restricted in the neighborhood

$$
\begin{aligned}
\tilde{\gamma}_{\sigma, l}^{-}(t, \tilde{x}) & =\left(x_{\sigma, 1}^{-}(t), x_{\sigma, 2}^{-}(t), x_{\sigma, 3}^{-}(t)\right), \\
\tilde{\gamma}_{\sigma, u}^{+}(t, \tilde{x}) & =\left(x_{\sigma, 1}^{+}(t), x_{\sigma, 2}^{+}(t), x_{\sigma, 3}^{+}(t)\right)
\end{aligned}
$$


where $x_{\sigma_{0}, 2}^{-}$can be assumed to increases monotonely for $t \in[-T, 0]$ and $x_{\sigma_{0}, 2}^{+}(t)>x_{2,0}$ for $t>0$. Since the unstable manifold is at least Lipschitz, these curves are Lipschitz in $\tilde{x}$. Since continuous function can be approximated by smooth functions, for small $\delta_{d}>0$, a tubular neighborhood of the semi-static curves $\left\{\left.\tilde{\gamma}_{\sigma_{0}, l}^{-}\right|_{[-T, 0]}\right\}$ admits smooth foliation of curves

$$
\tilde{\zeta}_{\tilde{x}}:(\tilde{x}, t) \in\left(D+d^{\prime}\right) \times[-T, 0] \rightarrow \mathbb{T}^{3}
$$

so that each semi-static curve $\tilde{\gamma}_{\sigma_{0}, l}^{-}(\cdot, \tilde{x})$ keeps $\delta_{d^{-}}$-close to $\zeta_{\tilde{x}}$ in the sense that $d\left(\zeta_{\tilde{x}}(t), \tilde{\gamma}_{\sigma_{0}, l}^{-}(t, \tilde{x})\right)<\delta_{d}$ for all $t \in[-T, 0]$. Here we use $\tilde{x}$ to emphasize that the point is on the disk $D+d^{\prime}$. The tubular neighborhood is defined by the form

$$
\mathbf{C}=\cup_{-T \leq t \leq 0}\left\{\tilde{\zeta}_{\tilde{x}}(t): \tilde{x} \in D+d_{1}\right\} .
$$

Let $\rho:\left(D+d^{\prime}\right) \times \mathbb{R} \rightarrow \mathbb{R}$ be a smooth function so that $\rho(\tilde{x}, t)=\rho\left(\tilde{x}^{\prime}, t\right)$ $\forall \tilde{x}, \tilde{x}^{\prime} \in D, \rho\left(\tilde{x}^{\prime}, t\right)=0$ if $t \notin[-T, 0]$ and $\rho(x, t)>0$ if $x \in D+d^{\prime}$ and $t \in(-T, 0)$. Since $\zeta_{\tilde{x}}$ is a smooth foliation of the tubular domain, it can be thought as a differeomorphism $\Psi:\left(D+d^{\prime}\right) \times[-T, 0] \rightarrow \mathbf{C}$, namely, for $\tilde{x}^{\prime} \in \mathbf{C}$ there exists a unique $(\tilde{x}, t) \in\left(D+d^{\prime}\right) \times[-T, 0]$ such that $\Psi(\tilde{x}, t)=\tilde{\zeta}_{\tilde{x}}(t)=\tilde{x}^{\prime}$. With a smooth function $V: D+d^{\prime} \rightarrow \mathbb{R}$ one obtains a smooth function $\tilde{V}$ supported on $\mathbf{C}$

$$
\tilde{V}\left(\tilde{x}^{\prime}\right)=\rho\left(\Psi^{-1}\left(\tilde{x}^{\prime}\right)\right) V(\tilde{x}) .
$$

By the construction of $\tilde{V}$, some constant $C_{3}>0$ exists such that

$$
\int_{-T}^{0} \tilde{V}\left(\tilde{\zeta}_{\tilde{x}}(t)\right) d t=C_{3} V(\tilde{x}), \quad \forall \tilde{x} \in D .
$$

The potential perturbation is constructed in the form of (4.12) where $V$ ranges over the function space spanned by

$$
\begin{aligned}
& \mathfrak{V}_{1}=\mu\left(\sum_{\ell=1,2} a_{\ell} \cos 2 \ell \pi\left(x_{1}-x_{1,0}\right)+b_{\ell} \sin 2 \ell \pi\left(x_{1}-x_{1,0}\right)\right), \\
& \mathfrak{V}_{3}=\mu\left(\sum_{\ell=1,2} c_{\ell} \cos 2 \ell \pi\left(x_{3}-x_{3,0}\right)+d_{\ell} \sin 2 \ell \pi\left(x_{3}-x_{3,0}\right)\right),
\end{aligned}
$$

each parameter of $\left(a_{\ell}, b_{\ell}, c_{\ell}, d_{\ell}\right)$ ranges over the unit interval [1,2].

Lemma 4.4. In the cube $[1,2]^{8}$ of the parameters, a set with Lebesgue measure not smaller than $1-O(\mu)$ exists so that for each $\left(a_{\ell}, b_{\ell}, c_{\ell}, d_{\ell}\right)$ in the set it holds simultaneously for all $\sigma \in \mathbb{I}_{\sigma_{0}}$ that 


$$
\left.\Pi_{i} \arg \min \left(u_{\sigma, l}^{-}-u_{\sigma, u}^{+}\right)\right|_{D} \subsetneq\left[x_{i, 0}-d, x_{i, 0}+d\right], \quad \forall, i=1,3,
$$

where $\mathbb{I}_{\sigma_{0}}$ is a neighborhood of $\sigma_{0}$, independent of $\mu$.

Proof. Recall that $u_{\sigma, u}^{+}$and $u_{\sigma, l}^{-}$denote the weak KAM solution generating forward semi-static orbits approaching $\tilde{\Gamma}_{\sigma, u}$ and backward semi-static orbits approaching $\tilde{\Gamma}_{\sigma, l}$ respectively. Let $u_{\sigma, u, \tilde{V}}^{+}$and $u_{\sigma, l, \tilde{V}}^{-}$denote the weak KAM solutions defined in the same way for the system under potential perturbation $L(\dot{x}, x) \rightarrow L(\dot{x}, x)+\tilde{V}(x)$ of $(4.12)$.

Under the potential perturbation, the cylinder remains unchanged. Restricted on the disk $D$, the function $u_{\sigma, r, \tilde{V}}^{+}$keeps the same as $u_{\sigma, r}^{+}$, but the function $u_{\sigma, \ell, \tilde{V}}^{-}$undergoes the perturbation $u_{\sigma, \ell, \tilde{V}}^{-} \neq u_{\sigma, \ell}^{-}$. If $\tilde{\gamma}_{\sigma, l, \tilde{V}}^{-}(\cdot, \tilde{x})$ is a semi-static curve produced by $u_{\sigma, \ell, \tilde{V}}^{-}$with $\tilde{\gamma}_{\sigma, l, \tilde{V}}^{-}(0, \tilde{x})=\tilde{x}$ and $\sigma \in \mathbb{I}_{\sigma_{0}}$, then

$$
\begin{aligned}
u_{\sigma, l, \tilde{V}}^{-}(\tilde{x})-u_{\sigma, l, \tilde{V}^{-}}^{-}\left(\tilde{\gamma}_{\sigma, l, \tilde{V}}^{-}(-t, \tilde{x})\right)= & \int_{-t}^{0}\left(L+\tilde{V}-\eta_{c(\sigma)}\right)\left(d \tilde{\gamma}_{\sigma, l, \tilde{V}^{-}}^{-}(-t, \tilde{x})\right) d t \\
& +\alpha(c(\sigma)) t \\
u_{\sigma, l, \tilde{V}^{\prime}}^{-}(\tilde{x})-u_{\sigma, l, \tilde{V}^{\prime}}^{-}\left(\tilde{\gamma}_{\sigma, l, \tilde{V}}^{-}(-t, \tilde{x})\right) \leq & \int_{-t}^{0}\left(L+\tilde{V}^{\prime}-\eta_{c(\sigma)}\right)\left(d \tilde{\gamma}_{\sigma, l, \tilde{V}^{-}}^{-}(-t, \tilde{x})\right) d t \\
& +\alpha(c(\sigma)) t .
\end{aligned}
$$

For large $t$ the curve $\tilde{\gamma}_{\sigma, l, \tilde{V}}^{-}(-t, \tilde{x})$ shall retreat into a small neighborhood of $\tilde{\Gamma}_{\sigma, l, \tilde{V}}$ where the function $u_{\sigma, l, \tilde{V}}^{-}$remains unchanged. So, it follows from the last two formulae that

$$
u_{\sigma, l, \tilde{V}}^{-}(\tilde{x})-u_{\sigma, l, \tilde{V}^{\prime}}^{-}(\tilde{x}) \geq \int_{-T}^{0}\left(\tilde{V}-\tilde{V}^{\prime}\right)\left(\tilde{\gamma}_{\sigma, l, \tilde{V}}^{-}(-t, \tilde{x})\right) d t
$$

In a similar way, we find

$$
u_{\sigma, l, \tilde{V}}^{-}(\tilde{x})-u_{\sigma, l, \tilde{V}^{\prime}}^{-}(\tilde{x}) \leq \int_{-T}^{0}\left(\tilde{V}-\tilde{V}^{\prime}\right)\left(\tilde{\gamma}_{\sigma, l, \tilde{V}^{\prime}}^{-}(-t, \tilde{x})\right) d t
$$

where $\tilde{\gamma}_{\sigma, l, \tilde{V}^{\prime}}^{-}$, denotes the backward semi-static curve produced by the function $u_{\sigma, l, \tilde{V}^{\prime}}^{-}$with $\tilde{\gamma}_{\sigma, l, \tilde{V}^{\prime}}^{-}(0, \tilde{x})=\tilde{x}$. Because $\tilde{x}$ lies in the region where $u_{\sigma, l, \tilde{V}}^{-}$ is differentiable, one has $\left|\tilde{\gamma}_{\sigma, l, \tilde{V}}^{-}(t, \tilde{x})-\tilde{\gamma}_{\sigma, l, \tilde{V}^{\prime}}^{-}(t, \tilde{x})\right| \rightarrow 0$ as $\left|\tilde{V}-\tilde{V}^{\prime}\right| \rightarrow 0$, guaranteed by the upper-semi continuity of semi-static curves. Therefore, for $\tilde{x} \in D$ one has 


$$
\begin{aligned}
u_{\sigma, l, \tilde{V}^{-}}^{-}(\tilde{x})-u_{\sigma, l, \tilde{V}^{\prime}}^{-}(\tilde{x}) & =\int_{-T}^{0}\left(\tilde{V}-\tilde{V}^{\prime}\right)\left(\tilde{\gamma}_{\sigma, l, \tilde{V}^{-}}^{-}(t, \tilde{x})\right) d t+o\left(\left\|\tilde{V}-\tilde{V}^{\prime}\right\|\right) \\
& =\left(\mathscr{K}_{\sigma}+\mathscr{R}_{\sigma}\right)\left(\tilde{V}-\tilde{V}^{\prime}\right)
\end{aligned}
$$

where the operator $\mathscr{K}_{\sigma}$ is linear

$$
\mathscr{K}_{\sigma} \tilde{V}(\tilde{x})=\int_{-T}^{0} \tilde{V}\left(\tilde{\gamma}_{\sigma, l, \tilde{V}}^{-}(t, \tilde{x})\right) d t=V(\tilde{x}) \int_{-T}^{0} \rho\left(\Psi^{-1}\left(\tilde{\gamma}_{\sigma, l, \tilde{V}}^{-}(t, \tilde{x})\right) d t\right.
$$

and $\mathscr{R}_{\sigma}\left(\tilde{V}-\tilde{V}^{\prime}\right)=o\left(\left\|V-V^{\prime}\right\|\right)$. As we introduce the perturbation in the way of (4.12), the operator $\mathscr{K}_{\sigma}$ can be thought as a linear map $V \rightarrow \mathscr{K}_{\sigma} V$, and one obtains from the formula just above that

$$
\mathscr{K}_{\sigma} V(\tilde{x})=A_{\sigma}(\tilde{x}) V(\tilde{x})
$$

where $\left|A_{\sigma}(\tilde{x})-C_{3}\right|$ is small if $\sigma$ is close to $\sigma_{0}$, and what is more important, the function $A_{\sigma}$ is $\frac{1}{2}$-Hölder continuous in $\sigma$, which follows from the $C^{r-2}$ smooth dependence of unstable fibers on base point, one of the results of the theorem of normally hyperbolic invariant manifold.

To defined a neighborhood $\mathbb{I}_{\sigma_{0}}$ of $\sigma_{0}$ such that Lemma 4.4 holds, we notice that there exists $B=B(d)<1$ such that for each

$$
\begin{aligned}
V= & \sum_{\ell=1,2}\left\{a_{\ell} \cos 2 \ell \pi\left(x_{1}-x_{1,0}\right)+b_{\ell} \sin 2 \ell \pi\left(x_{1}-x_{1,0}\right)\right. \\
& \left.+c_{\ell} \cos 2 \ell \pi\left(x_{3}-x_{3,0}\right)+d_{\ell} \sin 2 \ell \pi\left(x_{3}-x_{3,0}\right)\right\}
\end{aligned}
$$

one has

$$
\min _{\tilde{x} \in D}|V(\tilde{x})| \leq B \max _{\tilde{x} \in D}|V(\tilde{x})| .
$$

Let $B<B_{1}<1$, we defined a neighborhood $\mathbb{I}_{\sigma}$ of $\sigma_{0}$ such that for each $\sigma \in \mathbb{I}_{\sigma}$

1. the disk $D$ does not intersect $\pi \tilde{\Gamma}_{\sigma, \ell}+\delta$;

2. for $\tilde{\gamma}_{\sigma, l}^{-}(t, \tilde{x}), \tilde{\gamma}_{\sigma, u}^{+}(t, \tilde{x})$ in the coordinate presentation of (4.11), $x_{\sigma, 2}^{-}$ increases monotonely $\forall t \in[-T, 0]$ and $x_{\sigma, 2}^{+}(t)>x_{2,0} \forall t>0$;

3. $\min _{\tilde{x} \in D}\left|A_{\sigma}(\tilde{x})\right| \geq B_{1} \max _{\tilde{x} \in D}\left|A_{\sigma}(\tilde{x})\right|$ and $A_{\sigma}(\tilde{x})>\frac{3}{4} C_{3}$.

The number $B_{1}$ exists. When $\sigma=\sigma_{0}$, by choosing $\tilde{\zeta}_{\tilde{x}}$ close to $\tilde{\gamma}_{\sigma_{0}, l}^{-}(\cdot, \tilde{x})$ the quantity $\left|A_{\sigma}(\tilde{x})-C_{3}\right|$ can be arbitrarily small. The upper semi-continuity of semi-static curves guarantees the existence of this neighborhood. This neighborhood of $\sigma_{0}$ is associated with a neighborhood of $c(\sigma)$, denoted by $\mathbb{I}_{c}: c(\sigma) \in \mathbb{I}_{c} \Leftrightarrow \sigma \in \mathbb{I}_{\sigma}$. 
We construct a grid for the parameters $\left(a_{i}, b_{i}, c_{i}, d_{i}\right)$ by splitting the domain equally into a family of cubes and setting the size length by

$$
\Delta a_{i}=\Delta b_{i}=\Delta c_{i}=\Delta d_{i}=\mu
$$

there are as many as $\left[\mu^{-8}\right]$ cubes.

Since $\sigma \in \mathbb{I}_{\sigma_{0}}$, some constant $C_{4}>0$ exists such that for $V, V^{\prime} \in$ $\operatorname{Span}\left\{\mathfrak{V}_{1}, \mathfrak{V}_{3}\right\}$

$$
\begin{aligned}
\operatorname{Osc}_{\tilde{\in} D}\left(\mathscr{K}_{\sigma} V-\mathscr{K}_{\sigma} V^{\prime}\right) & =\max _{\tilde{x}, \tilde{x}^{\prime} \in D}\left|\mathscr{K}_{\sigma} V(\tilde{x})-\mathscr{K}_{\sigma} V^{\prime}(\tilde{x})\right| \\
& >\frac{3}{4} C_{3}\left(1-\frac{B}{B_{1}}\right) \operatorname{Osc}_{\tilde{x} \in D}\left(V-V^{\prime}\right)>C_{4} \mu \Delta .
\end{aligned}
$$

with $\Delta=\max \left\{\left|a_{\ell}-a_{\ell}^{\prime}\right|,\left|b_{\ell}-b_{\ell}^{\prime}\right|,\left|c_{\ell}-c_{\ell}^{\prime}\right|,\left|d_{\ell}-d_{\ell}^{\prime}\right|\right\}$.

We split the interval $\mathbb{I}_{\sigma}$ equally into $K_{\sigma}\left[\mu^{-2}\right]$ small intervals, denoted by $\left\{I_{\sigma, j}\right\}_{j \in \mathbb{J}_{\sigma}}$, where $K_{\sigma}=\left[L_{\sigma}\left(\frac{24 C_{2}}{C_{4}}\right)^{2}\right], L_{\sigma}$ is the length of $\mathbb{I}_{\sigma}, C_{2}$ is the constant appearing in (4.3) and $C_{4}$ appearing in (4.16). Let $\sigma_{j}$ be the middle point of $I_{j}$, corresponding to a barrier function $u_{\sigma_{j}, l}^{-}-u_{\sigma_{j}, u}^{+}$. We also split the interval $\mathbb{I}_{c}$ equally into $K_{\sigma}\left[\mu^{-1}\right]$ small intervals, denoted by $\left\{I_{c, j}\right\}_{j \in \mathbb{J}_{c}}$.

We assume that under a perturbation $L \rightarrow L+\tilde{V}_{j}$ with $\tilde{V}_{j}=\rho \Psi^{-1} V_{j}$ such that

$$
\operatorname{Osc}_{x \in D} \min _{x_{3}}\left(u_{\sigma_{j}, l, \tilde{V}_{j}}^{-}-u_{\sigma_{j}, u, \tilde{V}_{j}}^{+}\right)=0
$$

where $V_{j}=\mu\left(\sum_{\ell=1,2} a_{\ell, j} \cos 2 \ell \pi\left(x_{1}-x_{1,0}\right)+b_{\ell, j} \sin 2 \ell \pi\left(x_{1}-x_{1,0}\right)\right)$. Let us consider what change the barrier function undergoes if the Lagrangian is under further perturbation $\tilde{V}^{\prime}=\rho \Psi^{-1} V^{\prime}$ where $V^{\prime}$ is determined by the parameters $\left(a_{\ell}^{\prime}, b_{\ell}^{\prime}\right)$

$$
V^{\prime}=\mu\left(\sum_{\ell=1,2} a_{\ell}^{\prime} \cos 2 \ell \pi\left(x_{1}-x_{1,0}\right)+b_{\ell}^{\prime} \sin 2 \ell \pi\left(x_{1}-x_{1,0}\right)\right) .
$$

Using the formula (4.14) and noticing $u_{\sigma_{j}, u, \tilde{V}_{j}}^{+}=u_{\sigma_{j}, u, \tilde{V}^{\prime}}^{+}$we obtain the identity

$$
\begin{aligned}
& \left(u_{\sigma, l, \tilde{V}^{\prime}}^{-}-u_{\sigma, u, \tilde{V}^{\prime}}^{+}\right)-\left(u_{\sigma_{j}, l, \tilde{V}^{\prime}}^{-}-u_{\sigma_{j}, u, \tilde{V}^{\prime}}^{+}\right) \\
= & \left(u_{\sigma, l, \tilde{V}^{\prime}}^{-}-u_{\sigma_{j}, l, \tilde{V}^{\prime}}^{-}\right)-\left(u_{\sigma, u, \tilde{V}^{\prime}}^{+}-u_{\sigma_{j}, u, \tilde{V}^{\prime}}^{+}\right) \\
& +\left(u_{\sigma_{j}, l, \tilde{V}^{\prime}}^{-}-u_{\sigma_{j}, l, \tilde{V}_{j}}^{-}\right)-\left(u_{\sigma_{j}, u, \tilde{V}^{\prime}}^{+}-u_{\sigma_{j}, u, \tilde{V}_{j}}^{+}\right)
\end{aligned}
$$




$$
\begin{aligned}
= & \left(u_{\sigma, l, \tilde{V}^{\prime}}^{-}-u_{\sigma_{j}, l, \tilde{V}^{\prime}}^{-}\right)-\left(u_{\sigma, u, \tilde{V}^{\prime}}^{+}-u_{\sigma_{j}, u, \tilde{V}^{\prime}}^{+}\right) \\
& +\left(\mathscr{K}_{\sigma_{j}}+\mathscr{R}_{\sigma_{j}}\right)\left(\tilde{V}_{j}-\tilde{V}^{\prime}\right) .
\end{aligned}
$$

For each $\sigma \in I_{j}$, in virtue of the inequalities (4.3) the first term on the righthand-side of the identity is not bigger than $\frac{1}{3} C_{4} \mu^{2}$. For small $\left\|\tilde{V}_{j}-\tilde{V}^{\prime}\right\|$ one has $\left\|\mathscr{R}_{\sigma_{j}}\left(\tilde{V}_{j}-\tilde{V}^{\prime}\right)\right\|<\frac{1}{3}\left\|\mathscr{K}_{\sigma_{j}}\left(\tilde{V}_{j}-\tilde{V}^{\prime}\right)\right\|$. Note that $V^{\prime}, V_{j}$ are independent of $x_{3}$. If the parameters $\left(a_{\ell}^{\prime}, b_{\ell}^{\prime}\right)$ satisfy

$$
\max \left\{\left|a_{\ell, j}-a_{\ell}^{\prime}\right|,\left|b_{\ell, j}-b_{\ell}^{\prime}\right|\right\} \geq \mu
$$

we find from the identities, the condition (4.17) and the estimate (4.16) that

$$
\operatorname{Osc}_{\tilde{x} \in D} \min _{x_{3}}\left(u_{l, \sigma}^{-}-u_{u, \sigma}^{+}-\left(\mathscr{K}_{\sigma}+\mathscr{R}_{\sigma}\right) \tilde{V}^{\prime}\right) \geq \frac{1}{3} C_{4} \mu^{2}>0
$$

It implies that, for each small square of parameter $(\sigma, c(\sigma)) \in I_{\sigma, j} \times I_{c, j^{\prime}}$ we only need to cancel out at most $2^{4} \mu$-cubes from the grid for $\left\{\Delta a_{\ell}, \Delta b_{\ell}: \ell=\right.$ $1,2\}$ so that the formula (4.18) holds for the all other cubes. Let $j$ range over the set $\mathbb{J}_{\sigma}$ and $j^{\prime}$ range over the set $\mathbb{J}_{c}$ we obtain a set $\mathbf{S}_{1}^{c} \subset\left\{a_{\ell} \in\right.$ $\left.[1,2], b_{\ell} \in[1,2]: \ell=1,2\right\}$ with Lebesgue measure

$$
\operatorname{meas}_{1}^{c} \geq 1-2^{4} K_{\sigma} \mu
$$

such that the formula (4.18) holds for each $\left(a_{\ell}^{\prime}, b_{\ell}^{\prime}\right) \in \mathbf{S}_{1}^{c}$ and for each $\sigma \in \mathbb{I}_{\sigma}$.

Considering perturbations from $V^{\prime} \in \mathfrak{V}_{3}$, in the same way we can show that some set $\mathbf{S}_{3}^{c} \subset\left\{c_{\ell} \in[1,2], d_{\ell} \in[1,2]: \ell=1,2\right\}$ with Lebesgue measure

$$
\operatorname{meas}_{3}^{c} \geq 1-2^{4} K_{\sigma} \mu
$$

such that the formula

$$
\operatorname{Osc}_{x \in D} \min _{x_{1}}\left(u_{l, \sigma}^{-}-u_{u, \sigma}^{+}-\left(\mathscr{K}_{\sigma}+\mathscr{R}_{\sigma}\right) \tilde{V}^{\prime}\right)>0
$$

for each $\left(c_{\ell}^{\prime}, c_{\ell}^{\prime}\right) \in \mathbf{S}_{3}^{c}$ and each $\sigma \in \mathbb{I}_{\sigma}$.

Therefore, for each $\left(a_{\ell}, b_{\ell}, c_{\ell}, d_{\ell},\right) \in \mathbf{S}_{1}^{c} \times \mathbf{S}_{3}^{c}$, the formulae (4.18) and (4.19) implies

$$
\left.\Pi_{i} \arg \min \left(u_{\sigma, l}^{-}-u_{\sigma, u}^{+}\right)\right|_{D} \subsetneq\left[x_{i, 0}-d, x_{i, 0}+d\right], \quad \forall, i=1,3 .
$$

holds for all $\sigma \in \mathbb{I}_{\sigma_{0}}$. This completes the proof.

Notice the construction of potential perturbation, the function $\rho(\tilde{x})$ may approach infinity in $C^{r}$-topology with $r>1$. It does not make trouble be- 
cause the smallness of perturbation is guaranteed by setting arbitrarily small coefficient $\mu$.

The 2-torus $\mathrm{T}$ is divided into as many as $O\left(\left[d^{-2}\right]\right)$ squares like $D$. Notice that $\mu>0$ can be arbitrarily small. Applying Lemma 4.4 to each square, we find that there exists an open-dense set $\mathfrak{V}_{d}$ of potential perturbation, for each $V \in \mathfrak{V}_{d}$ that the diameter of each connected component of

$$
\left.\arg \min \left(u_{\sigma, l, \tilde{V}}^{-}-u_{\sigma, u, \tilde{V}}^{+}\right)\right|_{\mathrm{T}}
$$

is not larger than $2 d$.

Each section of torus $\mathrm{T}$ admits a hierarchy of partition into small disks $\cup_{j} D_{k j}$ so that the size length $d_{k} \rightarrow 0$ as $k \rightarrow \infty$, the intersection $\cap \cap_{k} \mathfrak{V}_{d_{k}}$ is a residual set. Therefore, we have proved Theorem 4.2 .

Since the section of torus $\mathrm{T}$ is chosen so that all semi-static curves $\tilde{\gamma}_{\sigma}^{ \pm}$ pass through the section, it follows from the lemma, the set $\arg \min \left(u_{\sigma, l}^{-}-\right.$ $\left.u_{\sigma, u}^{+}\right)(x, 0) \backslash(\mathcal{A}(c(\sigma))+\delta)$ is totally disconnected. Since the parameter $\sigma$ is restricted on a closed set in the line which can be covered by finitely many $\mathbb{I}_{\sigma_{i}}$, this property is also open-sense for all $\sigma$ under our consideration.

\section{Application to the problem of Arnold diffusion}

We apply Theorem 1.1 and 1.2 to the study of Arnold diffusion in nearly integrable Hamiltonian systems with three degrees of freedom, and establish a transition chain passing through a small neighborhood of double resonance. The Hamiltonian takes the form

$$
H(p, q)=h(p)+\epsilon P(p, q), \quad(p, q) \in \mathbb{R}^{n} \times \mathbb{T}^{n}
$$

where $\partial^{2} h(p)$ is positive definite, both $h$ and $P$ are $C^{r}$-differentiable with $r \geq 6$. In autonomous case, the positive definiteness is sufficient for the application of Mather theory. We may modify the Hamiltonian so that the condition of super-linear growth is satisfied while the energy level set $H^{-1}(E)$ remains in the domain where the Hamiltonian is unchanged. As each energy level set is compact, each solution of the Hamilton equation can be extended to the whole $t \in \mathbb{R}$.

For nearly integrable Hamiltonian systems with $n$-degrees of freedom, Arnold asked a notable question (cf. [A66])

Conjecture: The "general case" for a Hamiltonian system (5.1) with $n \geq 3$ is represented by the situation that for an arbitrary pair of neighborhood of tori $p=p^{\prime}, p=p^{\prime \prime}$, in one component of the level set $h(p)=h\left(p^{\prime}\right)$ there exists, for sufficiently small $\epsilon$, an orbit intersecting both neighborhoods. 
As the first step to answer the question, we search for normal hyperbolic invariant cylinder (NHIC) along resonant path. Once a NHIC is found, around which the system turns out to be a priori unstable, one can refer to [B08, CY1, CY2, DLS06, LC, Tr2] for the construction of diffusion orbits. In the system with three degrees of freedom, an irreducible integer vector $k^{\prime} \in \mathbb{Z}^{3} \backslash\{0\}$ determines a path

$$
\Gamma^{\prime}=\left\{p \in h^{-1}(E):\left\langle\partial h(p), k^{\prime}\right\rangle=0\right\} .
$$

To make things clearer, we introduce a symplectic coordinate transformation

$$
\mathfrak{M}: \quad q \rightarrow M^{t} q, \quad p \rightarrow M^{-t} p,
$$

where the matrix $M=\left(k^{\prime}, k_{2}, k_{3}\right)$ is made up by three integer vectors with $\operatorname{det} M=1$. In the new coordinates the frequency appears to be $\partial h(p)=$ $\left(0, \omega_{2}, \omega_{3}\right)$. If $\left(\omega_{2}, \omega_{3}\right)$ is a Diophantine frequency at a point $p$, around which there will be a piece of cylinder [Tr1, El] and for typical perturbation $P$, its size is independent of $\epsilon$ (cf. [B10]).

However, there are points along the path where another resonant condition exists. A point $p^{\prime \prime} \in \Gamma^{\prime}$ is called double resonant if $\exists$ another irreducible vector $k^{\prime \prime} \in \mathbb{Z}^{3} \backslash\{0\}$, independent of $k^{\prime}$, such that $\left\langle k^{\prime \prime}, \partial h\left(p^{\prime \prime}\right)\right\rangle=0$ holds as well. If we set $k_{2}=k^{\prime \prime}$ in the matrix $M$, the frequency in the new coordinates takes the form $\partial h\left(p^{\prime \prime}\right)=\left(0,0, \omega_{3}\right)$. There are many choices for $k_{3}$, we choose a $k_{3}$ so that $\left|k_{3}\right|$ is the smallest one.

In the following, the Hamiltonian of (5.1) is assumed to be under the coordinate transformation already. So one has $\partial h\left(p^{\prime \prime}\right)=\left(0,0, \omega_{3}\right)$.

To get the normal form around a double resonance, we introduce a coordinate transformation $\Phi_{\epsilon F}$ which is defined as the time- $2 \pi$-map $\Phi_{\epsilon F}=$ $\left.\Phi_{\epsilon F}^{t}\right|_{t=2 \pi}$ of the Hamiltonian flow generated by the function $\epsilon F(p, q)$. This function solves the homological equation

$$
\left\langle\frac{\partial h}{\partial p}\left(p^{\prime \prime}\right), \frac{\partial F}{\partial q}\right\rangle=-P(p, q)+Z(p, q)
$$

where

$$
Z(p, q)=\sum_{\ell \in \mathbb{Z}^{3}, \ell_{3}=0} P_{\ell}(p) e^{i\left(\ell_{1} q_{1}+\ell_{2} q_{2}\right)},
$$

in which $P_{\ell}$ represents the Fourier coefficient of $P, \ell=\left(\ell_{1}, \ell_{2}, \ell_{3}\right)$. Expanding $F$ into Fourier series and comparing both sides of the equation we obtain

$$
F(p, q)=\sum_{\ell \in \mathbb{Z}^{3}, \ell_{3} \neq 0} \frac{i P_{\ell}(p)}{\left\langle\ell, \partial h\left(p^{\prime \prime}\right)\right\rangle} e^{i\langle\ell, q\rangle} .
$$


Under the transformation $\Phi_{\epsilon F}$ we obtain a new Hamiltonian

$$
\begin{aligned}
\Phi_{\epsilon F}^{*} H= & h(p)+\epsilon Z(p, q)+\epsilon\left\langle\frac{\partial h}{\partial p}(p)-\frac{\partial h}{\partial p}\left(p^{\prime \prime}\right), \frac{\partial F}{\partial q}\right\rangle \\
& +\frac{\epsilon^{2}}{2} \int_{0}^{1}(1-t)\{\{H, F\}, F\} \circ \Phi_{\epsilon F}^{t} d t .
\end{aligned}
$$

Notice $\left|\left\langle\ell, \partial h\left(p^{\prime \prime}\right)\right\rangle\right|=\left|\ell_{3} \omega_{3}\right|, \omega_{3}=\partial_{3} h\left(p^{\prime \prime}\right) \neq 0$ since $h\left(p^{\prime \prime}\right)>\min h$. To solve Equation (5.3), we do not encounter the problem of small divisor.

The function $\Phi_{\epsilon F}^{*} H(p, q)$ determines its Hamiltonian equation

$$
\frac{d q}{d t}=\frac{\partial}{\partial p} \Phi_{\epsilon F}^{*} H, \quad \frac{d p}{d t}=-\frac{\partial}{\partial q} \Phi_{\epsilon F}^{*} H .
$$

For this equation we introduce another transformation (call it homogenization)

$$
\tilde{G}_{\epsilon}=\frac{1}{\epsilon} \Phi_{\epsilon F}^{*} H, \quad \tilde{y}=\frac{1}{\sqrt{\epsilon}}\left(p-p^{\prime \prime}\right), \quad \tilde{x}=q, \quad s=\sqrt{\epsilon} t,
$$

with $\tilde{x}=\left(x, x_{3}\right), \tilde{y}=\left(y, y_{3}\right), x=\left(x_{1}, x_{2}\right), y=\left(y_{1}, y_{2}\right)$. In the new canonical variables $(\tilde{x}, \tilde{y})$ and the new time $s$, Equation (5.4) turns out to be the Hamiltonian equation with the generating function as the following:

$$
\tilde{G}_{\epsilon}=\frac{1}{\epsilon}\left(h\left(p^{\prime \prime}+\sqrt{\epsilon} \tilde{y}\right)-h\left(p^{\prime \prime}\right)\right)-V(x)+\sqrt{\epsilon} \tilde{R}_{\epsilon}(\tilde{x}, \tilde{y}),
$$

where $V=-Z\left(p^{\prime \prime}, x\right)$ and

$$
\begin{aligned}
& \tilde{R}_{\epsilon}=\tilde{R}_{1}+\tilde{R}_{2}+\tilde{R}_{3}, \\
& \tilde{R}_{1}=\frac{1}{\sqrt{\epsilon}}\left[Z\left(p^{\prime \prime}+\sqrt{\epsilon} \tilde{y}, x\right)-Z\left(p^{\prime \prime}, x\right)\right], \\
& \tilde{R}_{2}=\frac{1}{\sqrt{\epsilon}}\left\langle\frac{\partial h}{\partial p}\left(p^{\prime \prime}+\sqrt{\epsilon} \tilde{y}\right)-\frac{\partial h}{\partial p}\left(p^{\prime \prime}\right), \frac{\partial F}{\partial q}\right\rangle, \\
& \tilde{R}_{3}=\frac{\sqrt{\epsilon}}{2} \int_{0}^{1}(1-t)\{\{H, F\}, F\} \circ \Phi_{\epsilon F}^{t} d t .
\end{aligned}
$$

To choose the neighborhood where we study the normal form, we notice the following two points.

1. there are finitely many double resonant points $\left\{p_{i}^{\prime \prime}\right\} \subset \Gamma^{\prime}$ such that $\Gamma^{\prime}$ is covered by the disks $\left\{\left\|p-p_{i}^{\prime \prime}\right\|<K_{i}^{-1} \epsilon^{\kappa^{\prime}}\right\}$, where $\kappa^{\prime}<\frac{1}{6}$, $K_{i} \leq K^{*} \epsilon^{-\frac{1}{3}\left(1-3 \kappa^{\prime}\right)}$ is the period of the double resonance at $p_{i}^{\prime \prime}$, namely, 
$K_{i} \partial h\left(p_{i}^{\prime \prime}\right) \in \mathbb{Z}^{3}$ and $K \partial h\left(p_{i}^{\prime \prime}\right) \notin \mathbb{Z}^{3}$ for any $K<K_{i}, K^{*}$ is a constant independent of $\epsilon$ (see Chapter 3 of [Lo]). Therefore, the size of each disk is between $O\left(\epsilon^{\frac{1}{7}}\right)$ and $O\left(\epsilon^{\frac{1}{3}}\right)$;

2. one is unable to apply the KAM technique in $K \sqrt{\epsilon}$-neighborhood of double resonance to obtain invariant cylinder, even with large $K>0$.

Therefore, we will study the normal form (5.6) in the domain

$$
\Omega_{\epsilon}=\left\{(\tilde{x}, \tilde{y}):|\tilde{y}| \leq \epsilon^{\kappa-\frac{1}{2}}, \tilde{x} \in \mathbb{T}^{3}\right\} \text {, with } 0<\kappa<\frac{1}{2}
$$

where the term $\left|\sqrt{\epsilon} \tilde{R}_{i}\right|_{C^{r-2}}$ is bounded by a small number of order $O(\sqrt{\epsilon})$ (for $i=1,2,3$ ). By introducing coordinate rescaling and translation

$$
\left(y, \frac{\sqrt{\epsilon}}{\omega_{3}} I\right)=\frac{1}{\sqrt{\epsilon}}\left(p-p^{\prime \prime}\right), \quad \theta=\frac{\sqrt{\epsilon}}{\omega_{3}} x_{3} .
$$

and expand $\tilde{G}_{\epsilon}$ in $O\left(\epsilon^{\kappa}\right)$ neighborhood of $p^{\prime \prime}$ we get a local expression

$$
\begin{aligned}
& \tilde{G}_{\epsilon}(x, y, I, \theta)=I+\frac{1}{2}\left\langle\tilde{B}\left(y, \frac{\sqrt{\epsilon}}{\omega_{3}} I\right),\left(y, \frac{\sqrt{\epsilon}}{\omega_{3}} I\right)\right\rangle \\
& \quad-V(x)+\sqrt{\epsilon} \tilde{R}_{h}\left(y, \frac{\sqrt{\epsilon}}{\omega_{3}} I\right)+\sqrt{\epsilon} \tilde{R}_{\epsilon}\left(x, \frac{\omega_{3}}{\sqrt{\epsilon}} \theta, p^{\prime \prime}+\left(\sqrt{\epsilon} y, \frac{\epsilon}{\omega_{3}} I\right)\right)
\end{aligned}
$$

where $\tilde{B}=\frac{\partial^{2} h}{\partial p^{2}}\left(p^{\prime \prime}\right)$ and term $\tilde{R}_{h}$ represents the following

$$
\frac{1}{\sqrt{\epsilon}^{3}}\left[h\left(p^{\prime \prime}+\left(\sqrt{\epsilon} y, \frac{\epsilon}{\omega_{3}} I\right)\right)-\left[h\left(p^{\prime \prime}\right)+\epsilon I+\frac{\epsilon}{2}\left\langle\tilde{B}\left(y, \frac{\sqrt{\epsilon}}{\omega_{3}} I\right),\left(y, \frac{\sqrt{\epsilon}}{\omega_{3}} I\right)\right\rangle\right]\right] .
$$

For $|y| \leq O\left(\epsilon^{\kappa-\frac{1}{2}}\right)$ and $|I| \leq O\left(\epsilon^{\kappa-1}\right)$, by direct calculation we see that both $\sqrt{\epsilon} \tilde{R}_{h}$ and $\sqrt{\epsilon} \tilde{R}_{\epsilon}$ are bounded by a quantity of order $O(\sqrt{\epsilon})$ in $C^{r-2}$-topology. By the expression of $\tilde{G}_{\epsilon}$ in (5.8) we find that $\partial_{I} \tilde{G}_{\epsilon}=1+O(\epsilon)$ holds in the region where $|y| \leq O\left(\epsilon^{\kappa-\frac{1}{2}}\right)$ and $|I| \leq O\left(\epsilon^{\kappa-1}\right)$ and get a solution of the equation $\tilde{G}_{\epsilon}(x, y, \bar{I}, \theta)=0$ by applying the implicit function theorem

$$
G_{\epsilon}\left(x, y, \frac{\omega_{3}}{\sqrt{\epsilon}} \theta\right)=\frac{1}{2}\langle B y, y\rangle-V(x)+\sqrt{\epsilon} R_{\epsilon}^{\prime}\left(x, y, \frac{\omega_{3}}{\sqrt{\epsilon}} \theta\right) .
$$

where $B$ is got from $\tilde{B}$ by eliminating the third row and the third column, the remainder $\sqrt{\epsilon} R_{\epsilon}^{\prime}$ is bounded by $O\left(\epsilon^{\kappa}\right)$ in $C^{r-2}$-topology with respect to the variable $\left(x, y, \frac{\omega_{3}}{\sqrt{\epsilon}} \theta\right)$ when it is restricted on the region $|y| \leq O\left(\epsilon^{\kappa-\frac{1}{2}}\right)$. 
Let $A=B^{-1}$, one obtains from the Hamiltonian $G_{\epsilon}$ the Lagrangian of (1.1) by the Legendre transformation. It allows us to apply Theorem 1.1 and 1.2 by keeping it in mind that all Mather, Aubry and Mañé sets are symplectic invariant [B07]. Let $\tilde{\alpha}_{H}, \tilde{\alpha}_{\Phi_{\epsilon F}^{*} H}$ and $\tilde{\alpha}_{\epsilon}$ be the $\alpha$-function for $H$, $\Phi_{\epsilon F}^{*} H$ and $\tilde{G}_{\epsilon}$ respectively.

The isoenergetic reduction from systems with three degrees of freedom to two and half induces a relation between $\tilde{\alpha}_{\epsilon}^{-1}(0)$ and the graph of $\alpha_{\epsilon}$ (cf. Theorem 3.3 and 3.4): if we regard the graph of $\alpha_{\epsilon}$ over $\mathbb{F}_{\epsilon} \cup \mathbb{A}_{\epsilon} \cup \mathbb{C}_{g} \cup \mathbb{C}_{g^{\prime}}$ as a set in $\mathbb{R}^{3}$,

$$
\left\{\left(\alpha_{\epsilon}(c), c\right): c \in \mathbb{F}_{\epsilon} \cup \mathbb{A}_{\epsilon} \cup \mathbb{C}_{g} \cup \mathbb{C}_{g^{\prime}}\right\}
$$

it precisely lies in the surface $\tilde{\alpha}_{\epsilon}^{-1}(0)$. We denote the corresponding parts by $\tilde{\mathbb{F}}_{\epsilon}, \tilde{\mathbb{A}}_{\epsilon}, \tilde{\mathbb{C}}_{g}$ and $\tilde{\mathbb{C}}_{g^{\prime}}$ respectively. Formula (5.2) induces a linear transformation in $H^{1}\left(\mathbb{T}^{3}, \mathbb{R}\right)$ under which the sphere $\tilde{\alpha}_{H}$ undergoes a linear transformation. Because of the rescaling (5.5) one has

$$
\int y d x-\Phi_{\epsilon F}^{*} H d t=\sqrt{\epsilon}\left(\int p d x-\tilde{G}_{\epsilon} d s\right)
$$

the rescaling (5.7) induces a rescaling from $\tilde{\alpha}_{\epsilon}^{-1}(0)$ to $\tilde{\alpha}_{\Phi_{\epsilon F}^{*} H}^{-1}(0)$

$$
c-c^{\prime} \rightarrow \sqrt{\epsilon}\left(c-c^{\prime}\right), \quad c_{3}-c_{3}^{\prime} \rightarrow \epsilon\left(c_{3}-c_{3}^{\prime}\right) .
$$

The Hamiltomorphism $\Phi_{\epsilon F}$ does not change the cohomology class.

Therefore, returning to the original coordinates, one has the following observation. The flat $\tilde{\mathbb{F}}_{\epsilon}$ is a disk with a size $O(\sqrt{\epsilon})$, the annulus $\tilde{\mathbb{A}}_{\epsilon}$ has a thickness of $\Delta_{V} \sqrt{\epsilon}$ and the channels $\tilde{\mathbb{C}}_{g}$ and $\tilde{\mathbb{C}}_{g^{\prime}}$ extend into the annulus, because of Theorem 4.1. Any two classes $\tilde{c} \in \tilde{\mathbb{C}}_{g}, \tilde{c}^{\prime} \in \tilde{\mathbb{C}}_{g^{\prime}}$ is connected by a transition chain if $c_{3}, c_{3}^{\prime} \leq \epsilon^{2 \kappa}$. See Figure 6 .

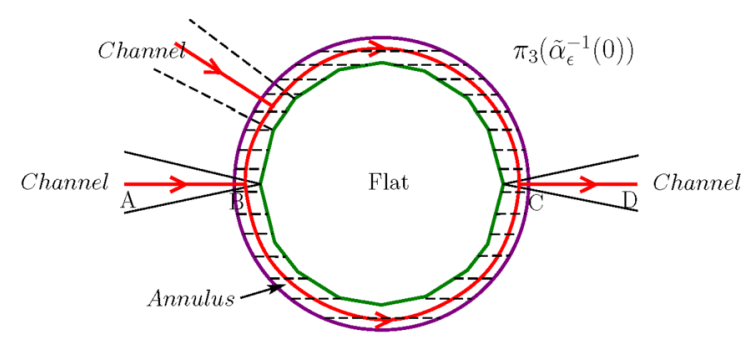

Figure 6: The transition chain under $\pi_{3}: \tilde{\alpha}_{\epsilon}^{-1}(0) \rightarrow \mathbb{R}^{2}$, represented by the thick solid red curve. Along the circle, $c_{3}$ keeps constant. 
Since the announcement of Mather (cf. [M03]), it has been widely known a difficult problem how to cross double resonance. Along the path suggested by Mather one has to consider the dynamics in the zero energy level ([KZ, Mar]). The path we figured out here is different, which does not touch the zero energy level. It turns out to be applicable even in the study of Arnold diffusion in nearly integrable systems with arbitrary degrees of freedom [CX]. In the available way to handle systems with more than three degrees of freedom, certain singularities arises at zero energy level when one makes reduction of order. It prevents one from touching the zero energy level.

Finally, let us consider the question raised by Arnold. Given any two $p, p^{\prime} \in h^{-1}(E)$ and any small $\delta>0$, there exist two irreducible integer vectors $k, k^{\prime} \in \mathbb{Z}^{3} \backslash\{0\}$ such that the circles of resonance $\Gamma_{k}=\left\{p \in \mathbb{R}^{3}\right.$ : $h(p)=E,\langle k, \partial h(p)\rangle=0\}, \Gamma_{k^{\prime}}=\left\{p \in \mathbb{R}^{3}: h(p)=E,\left\langle k^{\prime}, \partial h(p)\right\rangle=0\right\}$ passes through a $\delta$-neighborhood of $p$ and $p^{\prime}$ respectively. These two circles are either coincide or intersect at two points. In both cases, one obtains a reso-

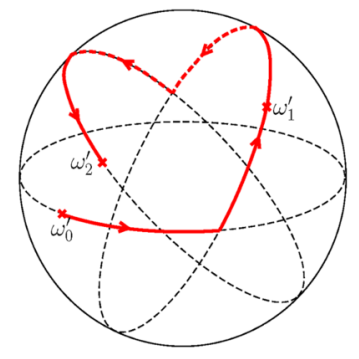

Figure 7: The resonant path in the surface of $h^{-1}(E)$.

nant path connecting the $\delta$-neighborhood of $p$ and $p^{\prime}$. This path is covered by finitely many disks $\left\{\left\|p-p_{i}^{\prime \prime}\right\|<K_{i}^{-1} \epsilon^{\kappa^{\prime}}\right\}$ where each $p_{i}^{\prime \prime}$ is a double resonant point the path. Although the number of the disks depends on $\epsilon$, the number of strong double resonance is independent of $\epsilon$ for generic perturbation $P$. A double resonance is called strong if there does not exist NHIC around. See Section 6 of [C15] for the quantitative criteria for distinguishing strong and weak double resonances.

To state the result we will obtain by applying Theorem 1.1 and 1.2 , we introduce some notations. For $E>0$, let $H^{-1}(E)=\{(p, q): H(p, q)=$ $E\}$ denote the energy level set, $B \subset \mathbb{R}^{3}$ denote a ball in $\mathbb{R}^{3}$ such that $\bigcup_{E^{\prime} \leq E+1} h^{-1}\left(E^{\prime}\right) \subset B$. Let $\mathfrak{S}_{a}, \mathfrak{B}_{a} \subset C^{r}\left(B \times \mathbb{T}^{3}, \mathbb{R}\right)(r \geq 6)$ denote a sphere and a ball, centered at the origin with radius $a>0$ respectively: $F \in \mathfrak{S}_{a}$ if and only $\|F\|_{C^{r}}=a$ and $F \in \mathfrak{B}_{a}$ if and only $\|F\|_{C^{r}} \leq a$. They inherit the topology from $C^{r}\left(B \times \mathbb{T}^{3}, \mathbb{R}\right)$. 
For perturbation $P$ independent of $p$ (classical mechanical system) we use the same notation $\mathfrak{S}_{a}, \mathfrak{B}_{a} \subset C^{r}\left(\mathbb{T}^{3}, \mathbb{R}\right)$ to denote a sphere and a ball with radius $a>0$

Let $\mathfrak{R}_{a}$ be a set residual in $\mathfrak{S}_{a}$, each $P \in \mathfrak{R}_{a}$ is associated with a set $R_{P}$ residual in the interval $\left[0, a_{P}\right]$ with $a_{P} \leq a$. A set $\mathfrak{C}_{a}$ is said to be cusp-residual in $\mathfrak{B}_{a}$ if

$$
\mathfrak{C}_{a}=\left\{\lambda P: P \in \mathfrak{R}_{a}, \lambda \in R_{P}\right\}
$$

Regarding the action variable $p$ and cohomology class $c$ as points in $\mathbb{R}^{3}$, one has

Theorem 5.1. Given any two small balls $B_{\delta}(p), B_{\delta}\left(p^{\prime}\right) \subset \mathbb{R}^{3}$, where $p, p^{\prime} \in$ $h^{-1}(E)$ with $E>0$ and small $\delta>0$, there exists a cusp-residual set $\mathfrak{C}_{\epsilon_{0}}$ such that for each $\epsilon P \in \mathfrak{C}_{\epsilon_{0}}$, there is a transition chain that connects the class $\tilde{c}$ to $\tilde{c}^{\prime}$ which satisfy the condition $\alpha(\tilde{c})=\alpha\left(\tilde{c}^{\prime}\right),|p-\tilde{c}|<\delta$ and $\left|p^{\prime}-\tilde{c}^{\prime}\right|<\delta$.

Proof. Let $\Gamma \subset \Gamma_{k} * \Gamma_{k^{\prime}} \subset h^{-1}(E)$ be a path that connects $B_{\delta}(p)$ to $B_{\delta}\left(p^{\prime}\right)$. For every point $p \in \Gamma_{k}, \Gamma_{k^{\prime}}$ the frequency satisfies the resonance relation $\langle\partial h(p), k\rangle=0$ with $k=k, k^{\prime}$ respectively. Let $p_{i} \in \Gamma$ be a double resonant point such that $K_{i} \partial h\left(p_{i}\right) \in \mathbb{Z}^{3}$ with $K_{i} \leq K^{*} \epsilon^{-\frac{1}{3}\left(1-3 \kappa^{\prime}\right)}, K^{*}>0$ is independent of $\epsilon$. One step of KAM iteration is carried out in the region $\mathbb{T}^{3} \times\left\|p-p_{i}\right\| \leq K_{i}^{-1} \epsilon^{\kappa^{\prime}}$, the potential $V$ at $p_{i}$ is obtained by averaging $P$ over resonant circle and the remainder $\tilde{R}_{\epsilon}$ loses two times of differentiablity. The whole path $\Gamma$ is covered by the disks centered at $p_{i}$ with radius $K_{i}^{-1} \epsilon^{\kappa^{\prime}}$.

Although the number of the points $\left\{p_{i}\right\}$ depends on $\epsilon$, the number of strong double resonant points is finite, independent of $\epsilon$ for $C^{r}$-generic $P$. We denote the point by $p_{i}^{\prime \prime}$ to specify the double resonance is strong. Indeed, if we expand $P$ into Fourier series, then

$$
Z(p, q)=Z_{k}(p,\langle k, q\rangle)+Z_{k, k_{i}}\left(p,\langle k, q\rangle,\left\langle k_{i}, q\right\rangle\right)
$$

where

$$
\begin{aligned}
Z_{k} & =\sum_{j \in \mathbb{Z} \backslash\{0\}} P_{j k}(p) e^{j\langle k, q\rangle i}, \\
Z_{k, k_{i}} & =\sum_{(j, l) \in \mathbb{Z}^{2}, l \neq 0} P_{j k+l k_{i}}(p) e^{\left(j\langle k, q\rangle+l\left\langle k_{i}, q\right\rangle\right) i} .
\end{aligned}
$$

As $\left|P_{k}\right|$ decrease fast as $|k|$ increases $\left|P_{k}\right| \leq O\left(|k|^{-r}\right)$, the term $Z_{k, k_{i}}$ is treated as a small perturbation to $Z_{k}$ for big $\left|k_{i}\right|$. That is why, for generic $Z_{k}$, the number of strong double resonances is independent of $\epsilon$. The double resonance at the point $\Gamma_{k} \cap \Gamma_{k^{\prime}}$ is strong. 
For a weak resonant point $p_{i}$, there exist NHICs and a channel

$$
\tilde{\mathbb{C}}_{i}=\tilde{\alpha}_{H}^{-1}(E) \cap \mathscr{L}_{\tilde{\beta}_{H}}\left(\cup \nu \partial h(p): \Gamma \cap\left\{\left|p-p_{i}\right| \leq K_{i}^{-1} \epsilon^{\kappa^{\prime}}\right\},|\nu-1| \leq O(\epsilon)\right)
$$

foliated by flats (intervals) such that for each $\tilde{c} \in \tilde{\mathbb{C}}_{i}$ the Aubry set $\tilde{\mathcal{A}}(\tilde{c})$ lies on the cylinders. It is in the situation of a priori unstable case. For $C^{r-2}$ generic remainder the condition $\left(\right.$ HA) holds for all $\tilde{c} \in \tilde{\mathbb{C}}_{i}$ so that $\tilde{\mathcal{A}}(\tilde{c})$ is a 2-dimensional torus. It shall be specified later what generic condition one has.

Around a strong double resonant point $p_{i}^{\prime \prime}$, one obtains a classical system $\frac{1}{2}\langle A \dot{x}, \dot{x}\rangle+V_{i}(x)$ where $x=\left(\langle k, q\rangle,\left\langle k_{i}, q\right\rangle\right)$ and $V_{i}=Z\left(p_{i}^{\prime \prime},\langle k, q\rangle,\left\langle k_{i}, q\right\rangle\right)$. For $C^{r}$-generic $V_{i}$ the conditions $(\mathbf{H 1}, \mathbf{2}, \mathbf{3})$ are satisfied. Then, there is an annulus of cohomology equivalence

$$
\tilde{\mathbb{A}}_{i}=\tilde{\alpha}_{H}^{-1}(E) \cap \mathscr{L}_{\tilde{\beta}_{H}}\left\{\cup \omega: 0<\left|\omega-\nu \partial h\left(p_{i}^{\prime \prime}\right)\right| \leq \Delta_{V_{i}} \sqrt{\epsilon},|\nu-1| \leq O(\epsilon)\right\}
$$

where $\Delta_{V_{i}}>0$ is the number obtained in Theorem 1.1. It connects two channels of single resonance

$$
\tilde{\mathbb{C}}_{i}^{-} \cup \tilde{\mathbb{C}}_{i}^{+}=\tilde{\alpha}_{H}^{-1}(E) \cap \mathscr{L}_{\tilde{\beta}_{H}}\left\{\cup \nu \partial h(p): \Gamma \cap\left\{\epsilon^{\frac{1}{2}+d} \leq\left|p-p_{i}^{\prime \prime}\right| \leq K_{i}^{-1} \epsilon^{\kappa^{\prime}}\right\}\right\} .
$$

Corresponding to the channels there are NHICs such that for each $\tilde{c} \in \tilde{\mathbb{C}}_{i}^{-} \cup$ $\tilde{\mathbb{C}}_{i}^{+}$the Aubry set lies on the NHICs (the main result of [C15]). Under further $C^{r-2}$-generic perturbation, the condition $(\mathbf{H A})$ holds for those $\tilde{c} \in \tilde{\mathbb{C}}_{i}^{-} \cup \tilde{\mathbb{C}}_{i}^{+}$ so that the Aubry set is a 2-dimensional torus. It is guaranteed by Theorem 1.2 .

In this way we obtain a transition chain passing through the channels and skirting around the strong double resonant points along circles of cohomology equivalence

$$
\Gamma_{c} \subset\left(\tilde{\alpha}_{H}^{-1}(E) \cap \tilde{\mathbb{C}}_{i}\right) \cup \tilde{\mathbb{A}}_{i} .
$$

where $\tilde{\mathbb{C}}_{i}=\mathscr{L}_{\tilde{\beta}_{H}}\left(\cup \nu \partial h(p): p \in \Gamma \backslash \cup\left\{\left|p-p_{i}^{\prime \prime}\right| \leq \epsilon^{\frac{1}{2}+d}\right\},|\nu-1| \leq O(\epsilon)\right)$.

Let us specify the generic condition on the perturbation $\epsilon P$. The path $\Gamma_{k}$ induces a decomposition of functions $P(p, q)=Z_{k}(p,\langle k, q\rangle)+P^{\prime}(p, q)$ according to the Fourier expansion. Since $Z_{k}$ is periodic in $q,\langle k, q\rangle$ can be treated as a scalar variable $x$ defined on $\mathbb{T}$. As $Z_{k}$ is $C^{r}$-smooth in $(p, q)$, regarding $Z_{k}$ as a function defined on $\mathbb{R}^{n} \times \mathbb{T}$, there is a residual set $\mathfrak{Z}_{k}$ of $C^{r}(B \times \mathbb{T}, \mathbb{R})$ such that for each $Z_{k} \in \mathfrak{Z}_{k}$ it holds simultaneously for all $p \in \Gamma$ that the maximal point of $Z_{k}$ in $\langle k, q\rangle$ is non-degenerate [CZ1]. In this case, the number of strong resonant points is independent of $\epsilon$. Each $Z_{k} \in \mathfrak{Z}_{k}$ is 
associated with a set $\mathfrak{P}_{Z_{k}}^{\prime}$ residual in the ball $\mathfrak{B}_{\epsilon_{Z_{k}}} \in C^{r}\left(B \times \mathbb{T}^{3}, \mathbb{R}\right) / C^{r}(B \times$ $\mathbb{T}, \mathbb{R}$ ) with radius $\epsilon_{Z_{k}}>0$. For each $\epsilon P^{\prime} \in \mathfrak{P}_{Z_{k}}^{\prime}$, the condition (HA) holds for all channels $\tilde{\mathbb{C}}_{i}$ of weak double resonance.

For each strong resonant condition, one obtains further decomposition of the perturbation $P(p, q)=V_{i}(q)+P^{\prime \prime}(p, q)$ where $V_{i}=Z\left(p_{i}^{\prime \prime},\langle k, q\rangle,\left\langle k_{i}, q\right\rangle\right)$ which is defined on $\mathbb{T}^{2}$. There exists a set $\mathfrak{V}_{i}$ residual in the unit ball $C^{r}\left(\mathbb{T}^{2}, \mathbb{R}\right)$ so that hypotheses $(\mathbf{H 1}, \mathbf{2}, \mathbf{3})$ hold for each $V_{i} \in \mathfrak{V}_{i}$, which is then associated with a set $\mathfrak{P}_{V_{i}}^{\prime \prime}$ residual in the ball $\mathfrak{B}_{\epsilon_{V_{i}}} \in C^{r}\left(B \times \mathbb{T}^{3}, \mathbb{R}\right) / C^{r}\left(\mathbb{T}^{2}, \mathbb{R}\right)$ with radius $\epsilon_{V_{i}}>0$. For each $\epsilon P^{\prime \prime} \in \mathfrak{P}_{V_{i}}^{\prime \prime}$, the condition (HA) holds for all channels around the strong double resonant points.

To get a set $\mathfrak{P}_{i}$ cusp-residual in $\mathfrak{B}_{1} \subset C^{r}\left(B \times \mathbb{T}^{3}, \mathbb{R}\right)$ such that each $\epsilon P \in \mathfrak{P}_{i}$ is associated with certain transition chain passing through $\tilde{\mathbb{C}}_{i}^{-} \cup$ $\tilde{\mathbb{A}}_{i} \cup \tilde{\mathbb{C}}_{i}^{+}$, we notice the decomposition $C^{r}\left(B \times \mathbb{T}^{3}, \mathbb{R}\right)=C^{r}\left(\mathbb{T}^{2}, \mathbb{R}\right) \oplus\left(C^{r}(B \times\right.$ $\left.\left.\mathbb{T}^{3}, \mathbb{R}\right) / C^{r}\left(\mathbb{T}^{2}, \mathbb{R}\right)\right)$. Let $\pi_{i}, \pi_{i}^{\perp}$ be the projection $C^{r}\left(B \times \mathbb{T}^{3}, \mathbb{R}\right) \rightarrow C^{r}\left(\mathbb{T}^{2}, \mathbb{R}\right)$, $C^{r}\left(B \times \mathbb{T}^{3}, \mathbb{R}\right) \rightarrow C^{r}\left(B \times \mathbb{T}^{3}, \mathbb{R}\right) / C^{r}\left(\mathbb{T}^{2}, \mathbb{R}\right)$ respectively.

For each $\pi_{i} P \in \mathfrak{V}_{i}$, there is a set $\mathfrak{P}_{\pi_{i} P}^{\prime \prime}$ residual in $\mathfrak{B}_{\epsilon_{\pi_{i} P}} \subset C^{r}(B \times$ $\left.\mathbb{T}^{3}, \mathbb{R}\right) / C^{r}\left(\mathbb{T}^{2}, \mathbb{R}\right)$ with radius $\epsilon_{\pi_{i} P}>0$ such that for each $\epsilon \pi_{i}^{\perp} P \in \mathfrak{P}_{\pi_{i} P}^{\prime \prime}$ there is a transition chain to pass through $\tilde{\mathbb{C}}_{i}^{-} \cup \tilde{\mathbb{A}}_{i} \cup \tilde{\mathbb{C}}_{i}^{+}$. We extend $\mathfrak{P}_{\pi_{i} P}^{\prime \prime}$ to a set $\overline{\mathfrak{P}}_{\pi_{i} P}^{\prime \prime}$ residual in the unit ball $\mathfrak{B}_{1}$ of the quotient space $C^{r}(B \times$ $\left.\mathbb{T}^{3}, \mathbb{R}\right) / C^{r}\left(\mathbb{T}^{2}, \mathbb{R}\right)$ in whatever a way provided its restriction on the ball $\mathfrak{B}_{\epsilon}$ is the same as the original one. Let $\overline{\mathfrak{P}}_{i}=\left\{V_{i}+P^{\prime \prime}: V_{i} \in \mathfrak{V}_{i}, P^{\prime \prime} \in \overline{\mathfrak{P}}_{\pi_{i} P}^{\prime \prime}\right\}$, which is obviously residual in $\mathfrak{B}_{1} \subset C^{r}\left(B \times \mathbb{T}^{3}, \mathbb{R}\right)$. As $\mathfrak{B}_{1}=[0,1] \times \mathfrak{S}_{1}$, by applying the Kuratowski-Ulam theorem (categorical analogue of the Fubini theorem, c.f. Chapter 12 of $[\mathrm{Ox}]$ ), there exists a set $\mathfrak{R}$ residual in $\mathfrak{S}_{1}$ such that for each $P \in \Re$, there is set of numbers $R_{P}$ residual in $[0,1]$ such that $\lambda P \in \overline{\mathfrak{P}}_{i}$ for all $\lambda \in R_{P}$.

Indeed, $P \in \mathfrak{R}$ implies $\pi_{i} P \in \mathfrak{V}_{i}$. The set $\mathfrak{V}_{i}$ is invariant for the rescaling $V \rightarrow \lambda V$. Under the rescaling $y \rightarrow \sqrt{\nu} y, t \rightarrow \sqrt{\nu}^{-1} t$ in the classical system, the Hamiltonian equation for $\frac{1}{2}\langle A y, y\rangle+\nu V(x)$ is the same as it for $\frac{1}{2}\langle A y, y\rangle+$ $V(x)$. It follows from the way to extend $\mathfrak{P}_{\pi_{i} P}^{\prime \prime}$ that some number $a_{P}>0$ exists such that $\lambda \pi_{i}^{\perp} P \in \mathfrak{P}_{\pi_{i} P}^{\prime \prime}$ if $\lambda \in R_{P} \cap\left(0, a_{P}\right]$.

The generic property of transition chain passing through channel of weak double resonance is obtained in similar arguments.

With a transition chain connecting $\tilde{c}$ to $\tilde{c}^{\prime}$, one is able to construct orbit which connects $\tilde{\mathcal{A}}(\tilde{c})$ to $\tilde{\mathcal{A}}\left(\tilde{c}^{\prime}\right)$ by the variational method developed in [CY1, CY2, LC]. For nearly integrable Hamiltonian systems, along each orbit $(p(t), q(t))$ in $\tilde{\mathcal{A}}(\tilde{c})$ (the counterpart of the Aubry set in the cotangent bundle) one has $|p(t)-\tilde{c}| \ll 1$.

The conjecture of Arnold diffusion for positive definite Hamiltonian turns out be a theorem for $n=3$. 


\section{Construction of topological transitive orbits for $L_{0}$}

The main task of this section is to prove Theorem 1.3. Let $\delta_{i} \downarrow 0$ be a sequence of numbers. Since $L_{0}$ is autonomous, the set $\left\{\tilde{\mathcal{A}}(c): \alpha_{0}(c)=E\right\}$ is compact, for each $\delta_{i}>0$ there are finitely many cohomology classes $\left\{c_{i, j}\right\}_{1 \leq j \leq j_{i}}$ so that the set $\cup_{j} \tilde{\mathcal{A}}\left(c_{i, j}\right)$ is $\delta_{i}$-dense in the set $\left\{\tilde{\mathcal{A}}(c): \alpha_{0}(c)=E\right\}$ : for each $c \in \alpha_{0}^{-1}(E)$ there exists some $c_{i, j}$ such that $d\left(\tilde{\mathcal{A}}(c), \tilde{\mathcal{A}}\left(c_{i, j}\right)\right)<\delta_{i}$. As $\alpha_{0}^{-1}(E)$ is a circle, for each $i$, the classes $\left\{c_{i, j}\right\}$ are arranged in clockwise order. The $\delta_{i}$ is chosen so small such that $\tilde{\mathcal{A}}\left(c_{i, j}\right)$ is dynamically connected to $\tilde{\mathcal{A}}\left(c_{i, j+1}\right)$ modulo $j_{i}$ by the orbit constructed in Lemma 3.1.

As we are working on $\mathbb{T}^{2}$, the Fenchel-Legendre dual $\omega(c)=\mathscr{L}_{\beta_{0}}^{-1}(c)$ of $c \in \alpha_{0}^{-1}(E)$ is a point if $E>\min \alpha_{0}$, and there are coordinates so that $\omega_{1}(c)>0$ if we write it in coordinates $\omega(c)=\left(\omega_{1}(c), \omega_{2}(c)\right)$. Indeed, one can divide the circle $\alpha_{0}^{-1}(E)$ into three parts $\left\{\Gamma_{m}\right\}_{m=1,2,3}$, each $\Gamma_{m}$ is associated with a coordinate system $x \rightarrow M_{m} x$ where $\omega_{1}(c)>0$ holds $\forall c \in \Gamma_{m}$. For each $i \geq 0$, the set $\left\{c_{i, j}\right\}_{1<j \leq j_{i}}$ is automatically divided into three parts $\mathbb{E}_{m}$, $\mathbb{E}_{1}=\left\{c_{i, j}\right\}_{1 \leq j \leq j_{1}}, \mathbb{E}_{2}=\left\{c_{i, j}\right\}_{j_{1}+1 \leq j \leq j_{2}}$ and $\mathbb{E}_{3}=\left\{c_{i, j}\right\}_{j_{2}+1 \leq j \leq j_{i}}$. Since $c_{i, j}$ is assumed close to $c_{i, j+1}$, it is reasonable to assume $\omega_{1}\left(c_{i, j_{m}+1}\right)>0$ in both coordinates $M_{m} x$ and $M_{m+1} x$ for $m=1,2$.

Let $\Sigma_{a}=\left\{x \in \mathbb{T}^{2}, x_{1}=a\right\}$. For $c \in \alpha_{0}^{-1}(E)$ with $E>\min \alpha_{0}$, if $\mathcal{N}(c) \subsetneq$ $\mathbb{T}^{2}$ and the coordinate system is chosen such that $\omega_{1}>0$, then $\mathcal{N}(c) \cap \Sigma_{a}$ is topologically trivial, finitely many disjoint closed intervals $\left\{I_{c, a, \ell}\right\} \subset \mathbb{T}$ exist so that $\mathcal{N}(c) \cap \Sigma_{a} \subset \operatorname{int} \cup_{\ell} I_{c, a, \ell}$ by regarding each interval $I_{c, a, \ell}$ as an embedded segment in the circle $\Sigma_{a}$.

For each $c_{i, j}$ and in the associated coordinate system so that $\omega_{1}\left(c_{i, j}\right)>0$ we work in the covering space $\mathbb{R} \times \mathbb{T}$. The Aubry and Mañé sets have their natural lift to the covering space, for which we use the same notation. Let $\mu_{i, j}$ be a closed 1-form such that $\left[\mu_{i, j}\right]=c_{i, j+1}-c_{i, j}$ and its support does not touch the set $[-d, d] \times \cup I_{c_{i, j}, 0, \ell}$. The number $d>0$ is chosen suitably small so that $\mathcal{N}\left(c_{i, j}\right) \cap\left\{\left|x_{1}\right| \leq d\right\} \subset[-d, d] \times \cup I_{c_{i, j}, 0, \ell}$.

Recall what we did in the proof of Lemma 3.1. We regard the Lagrangian $L_{0}$ and the 1-form as that defined on the covering space, just think it is periodic in $x_{1}$. As before, let $\bar{x}=\left(\bar{x}_{1}, \bar{x}_{2}\right)$ denote a point in $\mathbb{R} \times \mathbb{T}$. Let $\rho_{i, j}$ : $\bar{M} \rightarrow \mathbb{R}$ be a smooth function such that $\rho_{i, j}=1$ for $\bar{x}_{1} \geq d, \rho_{i, j}=0$ for $\bar{x}_{1} \leq-d$ and let $\eta_{i, j}$ be a closed 1-form such that $\left[\eta_{i, j}\right]=c_{i, j}$. We define the Lagrangian $\bar{L}_{i, j}: T(\mathbb{R} \times \mathbb{T}) \rightarrow \mathbb{R}$

$$
\bar{L}_{i, j}=L_{0}+E-\eta_{i, j}-\rho_{i, j} \mu_{i, j} .
$$

The lift of the section $\Sigma_{0}$ to the covering space contains infinitely components, denoted by $\left\{\Sigma_{i}: \bar{x}_{1}=i\right\}$. Let $\bar{M}^{+}=\left\{\bar{x}: \bar{x}_{1} \geq 1\right\}$ and $\bar{M}^{-}=\left\{\bar{x}: \bar{x}_{1} \leq\right.$ 
$-1\}$. For $\bar{x} \in \bar{M}^{-}$and $\bar{x}^{\prime} \in \bar{M}^{+}$, let $\bar{\gamma}_{i, j}\left(\cdot, \bar{x}, \bar{x}^{\prime}\right):\left[-t\left(\bar{x}, \bar{x}^{\prime}\right), t\left(\bar{x}, \bar{x}^{\prime}\right)\right] \rightarrow \mathbb{R} \times \mathbb{T}$ be the minimal curve of the action

$$
A_{i, j}\left(\left.\bar{\gamma}_{i, j}\left(\cdot, \bar{x}, \bar{x}^{\prime}\right)\right|_{\left[-t\left(\bar{x}, \bar{x}^{\prime}\right), t\left(\bar{x}, \bar{x}^{\prime}\right)\right]}\right)=\inf _{t>0} \inf _{\substack{\bar{\gamma}(-t)=\bar{x} \\ \bar{\gamma}(t)=\bar{x}^{\prime}}} A_{i, j}(\bar{\gamma}),
$$

where

$$
A_{i, j}\left(\left.\bar{\gamma}\right|_{[-t, t]}\right)=\int_{-t}^{t} L_{i, j}(\bar{\gamma}(s), \dot{\bar{\gamma}}(s), s) d s .
$$

One has $t\left(\bar{x}, \bar{x}^{\prime}\right) \rightarrow \infty$ as $\bar{x}_{1}^{\prime}-\bar{x}_{1} \rightarrow \infty$, guaranteed by the super-linear growth of $L_{0}$ in $\dot{x}$ and $\omega_{1}>0$ (Lemma 2.1 in [LC]).

Lemma 6.1. Assume $\mathcal{N}\left(c_{i, j}\right) \subsetneq \mathbb{T}^{2}$ and $c_{i, j+1}$ is sufficiently close to $c_{i, j}$. Given $a>0$, there exists large $a^{\prime}>$ a such that, for the points $\bar{x}, \bar{x}^{\prime} \in \mathbb{R} \times \mathbb{T}$ with $\bar{x}_{1}<-a^{\prime}$ and $\bar{x}_{1}^{\prime}>a^{\prime}$, the minimal curve $\bar{\gamma}_{i, j}\left(\cdot, \bar{x}, \bar{x}^{\prime}\right)$ crosses the section $\Sigma_{a^{*}}=\left\{\bar{x} \in \mathbb{R} \times \mathbb{T}: \bar{x}_{1}=a^{*} \leq a\right\}$ by passing through the interior of $\cup I_{c_{i, j}, a^{*}, \ell}$ which are thought to be segments embedded in the circle $\Sigma_{a^{*}}$.

Proof. Recall the construction of $\mathscr{C}_{\bar{L}_{i, j}}$. Let $\bar{x}_{1} \rightarrow-\infty, \bar{x}_{1}^{\prime} \rightarrow \infty$, as it was shown in the proof of Lemma 3.1, each accumulation point of these minimal curves precisely falls into the set $\mathscr{C}_{\bar{L}_{i, j}}$, with which the set $\mathcal{C}_{\bar{L}_{i, j}}$ is defined in (3.2). Because the Lagrangian is autonomous, we eliminate the component $t$, namely, $\mathcal{C}_{\bar{L}_{i, j}}=\cup_{\bar{\gamma} \in \mathscr{C}_{\bar{L}_{i, j}}} \bar{\gamma}(t)$. Because of the upper semi-continuity $\bar{L}_{i, j} \rightarrow$ $\mathcal{C}_{\bar{L}_{i, j}}$, one has $\mathcal{C}_{\bar{L}_{i, j}} \subset \mathcal{N}\left(c_{i, j}\right)+\varepsilon$ provided $c_{i, j+1}$ is sufficiently close to $c_{i, j}$.

Restricted on the set $\left\{\bar{x}: \bar{x}_{1} \in[-a, a]\right\}$, all curves in $\mathscr{C}_{\bar{L}_{i, j}}$ are approximated by the minimal curves $\left\{\left.\bar{\gamma}_{i, j}\left(\cdot, \bar{x}, \bar{x}^{\prime}\right)\right|_{\bar{x}_{1} \in[-a, a]}\right\}$ as $-\bar{x}_{1}, \bar{x}_{1} \rightarrow \infty$, and the restriction of curves $\left\{\bar{\gamma}_{i, j}\left(\cdot, \bar{x}, \bar{x}^{\prime}\right)\right\}$ on the set $\left\{\bar{x}: \bar{x}_{1} \in[-a, a]\right\}$ take the restriction of curves in $\mathscr{C}_{\bar{L}_{i, j}}$ as their accumulation points. Therefore, for sufficiently large $a^{\prime}>a$ the lemma holds.

As the first step to construct transitive orbits, let us construct orbits which visit $\delta_{i}$-neighborhood of $\tilde{\mathcal{A}}\left(c_{i, j}\right)$ for all $c_{i, j} \in \Gamma_{1}$. In this case, there is a coordinate system where one has $\omega_{1}\left(c_{i, j}\right)>0$ for all classes $c_{i, j} \in \Gamma_{1}$, where $j=1,2, \cdots j_{1}$. In the covering space $T(\mathbb{R} \times \mathbb{T})$, we introduce a modified Lagrangian

$$
\tilde{L}_{i, 1}=L_{0}+E-\eta_{i, 1}-\sum_{j=1}^{j_{1}-1}\left(k_{i, j}^{*} \rho_{i, j}\right) \mu_{i, j}
$$

where $k_{i, 1}<\cdots<k_{i, j_{1}}$ is a sequence of positive integers, each $k_{i, j}$ induces an operation on functions $k_{i, j}^{*} \rho(\bar{x})=\rho\left(\bar{x}_{1}-k_{i, j}, \bar{x}_{2}\right)$. Let $\bar{x}, \bar{x}^{\prime}$ be the points such that $\bar{x}_{1}<-k_{i, 1}$ and $\bar{x}_{1}^{\prime}>k_{i, j_{1}}$ and let $\bar{\gamma}_{i, 1}\left(\cdot, \bar{x}, \bar{x}^{\prime}\right):\left[-t\left(\bar{x}, \bar{x}^{\prime}\right), t\left(\bar{x}, \bar{x}^{\prime}\right)\right] \rightarrow$ $\mathbb{R} \times \mathbb{T}$ be the minimal curve of the action 


$$
A_{\tilde{L}_{i, 1}}\left(\bar{\gamma}_{i, 1}\left(\cdot, \bar{x}, \bar{x}^{\prime}\right)\right)=\inf _{\substack{t>0 \\ \inf _{\bar{\gamma}(-t)=\bar{x}}(t)=\bar{x}^{\prime}}} A_{\tilde{L}_{i, 1}}(\bar{\gamma})
$$

where

$$
A_{\tilde{L}_{i, 1}}(\bar{\gamma})=\int_{-t}^{t} \tilde{L}_{i, 1}(\bar{\gamma}(s), \dot{\bar{\gamma}}(s), s) d s .
$$

We claim that for sufficiently large $k_{i, 1}>0$ and $k_{i, j+1}-k_{i, j}>0$ with $j=2, \cdots, j_{1}-1$,

1. the curve $\bar{\gamma}_{i, 1}\left(\cdot, \bar{x}, \bar{x}^{\prime}\right)$ crosses the section $\Sigma_{k_{i, j}}$ by passing a collection of disjoint intervals $\cup I_{c_{i, j}, 0, \ell} \subset \Sigma_{k_{i, j}}$ and as an immediate consequence, it solves the Euler-Lagrange equation determined by $L_{0}$;

2. for any small $\delta_{i}>0$, the orbit $\left(\bar{\gamma}_{i, 1}, \dot{\bar{\gamma}}_{i, 1}\right)$ generated by the curve $\bar{\gamma}_{i, 1}\left(\cdot, \bar{x}, \bar{x}^{\prime}\right)$ visits the $\delta_{i}$-neighborhood of every Aubry set $\tilde{\mathcal{A}}\left(c_{i, j}\right)$ for $j=1,2, \cdots, j_{1}$.

To verify the first one, we apply Lemma 6.1 . Let $\bar{x}^{1}$ be the intersection point of the curve $\bar{\gamma}_{i, 1}\left(\cdot, \bar{x}, \bar{x}^{\prime}\right)$ with the section $\Sigma_{k_{1}}$. The restriction of $\bar{\gamma}_{i, 1}\left(\cdot, \bar{x}, \bar{x}^{\prime}\right)$ to the segment joining the point $\bar{x}$ with $\bar{x}^{1}$ is obviously a minimal curve. By applying Lemma 6.1 we find that the term $\rho_{i, 1} \mu_{i, 1}$ does not contribute to the Euler-Lagrange equation, namely, the conclusion holds for $j=1$ if $k_{i, 1}$ is sufficiently large. The same argument applies to $j=2,3, \cdots, j_{1}-1$ if all numbers $\left\{k_{i, j+1}-k_{i, j}\right\}$ are sufficiently large.

To check the second, we notice that, restricted on the cylinder $\{\bar{x}$ : $\left.\bar{x}_{1} \in\left[k_{i, j}+1, k_{i, j+1}\right]\right\}$, the projection of the minimal curve $\bar{\gamma}_{i, 1}\left(\cdot, \bar{x}, \bar{x}^{\prime}\right)$ is indeed $c_{i, j+1}$-minimal for the Lagrangian $L_{0}$ with given boundary condition. So, along the segment of this orbit, there must be points approaching $\tilde{\mathcal{A}}(c)$ provided $k_{i, j+1}-k_{i, j}>0$ is sufficiently large. Otherwise this orbit would approach the support of some invariant measure which is not $c_{i, j+1}$-minimal.

As the second step, we study the problem whether there are orbits of the Lagrangian flow $\phi_{L_{0}}^{t}$ which visit $\delta_{i}$-neighborhood of each Aubry set $\tilde{\mathcal{A}}\left(c_{i, j}\right)$ for $j=1,2, \cdots, j_{i}$. Apparently, the result we just obtained also applies for all $c_{i, j} \in \Gamma_{m}$ with $m=2,3$. We need to handle the problem how to match the orbits we obtained in different coordinate systems.

Assume the Lagrangian $L_{0}$ takes the form in the coordinate system so that $\omega_{1}(c)>0$ for all $c \in \Gamma_{1}$. For $c \in \Gamma_{m}$ with $m=2,3$ we need to introduce a linear coordinate transformation $\Phi_{m}: x \rightarrow M_{m} x$. Let $\Phi_{m}^{*} L_{0}$ denote the Lagrangian $L_{0}\left(M_{m} \dot{x}, M_{m} x\right)$, i.e. $\Phi_{m}^{*} L_{0}(\dot{x}, x)=L_{0}\left(M_{m} \dot{x}, M_{m} x\right)$.

For $\mathbb{T}^{2}$, let $\Sigma_{0}=\left\{x_{1}=0\right\}$ be a section in the original coordinates. To the covering space $\mathbb{R} \times \mathbb{T}$ in the new coordinates $M_{2} x$, the lift of $\Sigma_{0}$ looks like a spiral line, extending to $\bar{x}_{1} \rightarrow \pm \infty$. Let $\bar{\Sigma}_{0}^{n}$ be a segment of the spiral line in the new coordinates such that the projection from $\bar{\Sigma}_{0}^{n} \rightarrow \Sigma_{0}$ is one 
to one. Let $\bar{\Sigma}_{0}^{o}$ be a connected component in the lift of $\Sigma_{0}$ in the original coordinates. The superscript $n$, o means new, old respectively.

Since $\omega_{1}\left(c_{i, j_{1}}\right)>0$ in both coordinates and $\mathcal{N}\left(c_{i, j_{1}}\right) \subsetneq \mathbb{T}^{2}$, the set $\mathcal{N}\left(c_{i, j_{1}}\right) \cap \Sigma_{0}$ is covered by finitely many disjoint closed intervals $\cup I_{c_{i, j_{1}}, 0, \ell}$. Denoted by $\cup \bar{I}_{c_{i, j_{1}}, 0, \ell}^{n}$ as a part in the lift of $\cup I_{c_{i, j_{1}}, 0, \ell}$ to $\mathbb{R} \times \mathbb{T}$ in the new coordinates and by $\cup \bar{I}_{c_{i, j_{1}}, 0, \ell}^{o}$ as a part in the lift in the older coordinates such that

1. the projection from $\cup \bar{I}_{c_{i, j_{1}}, 0, \ell} \subset \bar{\Sigma}_{0}^{\imath}$ to $\cup I_{c_{i, j_{1}+1}, 0, \ell}$ is one to one for $\imath=n, o$;

2. $\bar{x}_{1}<-k_{i, j_{1}} \forall \bar{x} \in \cup \bar{I}_{c_{i, j_{1}}, 0, \ell}^{n}$ and $\bar{x}_{1} \geq k_{i, j_{1}} \forall \bar{x} \in \cup \bar{I}_{c_{i, j_{1}}, 0, \ell}^{o}$.

The same principle applies to the transition from $\mathbb{E}_{2}$ to $\mathbb{E}_{3}$. In the coordinates $M_{2} x$, one also has $\mathcal{N}\left(c_{i, j_{2}+1}\right) \cap \Sigma_{0} \subset \cup I_{c_{i, j_{2}+1}, 0, \ell}$ where $\Sigma_{0}=\left\{x_{1}=0\right\}$. These two properties hold if the subscript $j_{1}$ is replaced with $j_{2}$.

To construct orbits of $\phi_{L_{0}}^{t}$ visiting $\delta_{i}$-neighborhood of $\tilde{\mathcal{A}}\left(c_{i, j}\right)$ for all $j=$ $1,2, \cdots, j_{i}$, we introduce the Lagrangian

$$
\tilde{L}_{i, 2}=\Phi_{2}^{*} L_{0}+E-\eta_{i, j_{1}}-\sum_{j=j_{1}}^{j_{2}-1}\left(\left(k_{i, j}-k_{i, j_{1}}\right)^{*} \rho_{i, j}\right) \mu_{i, j},
$$

defined in the covering space $\bar{\pi}: \mathbb{R} \times \mathbb{T} \rightarrow \mathbb{T}^{2}$ in the coordinates $M_{2} x$ and the Lagrangian

$$
\tilde{L}_{i, 3}=\Phi_{3}^{*} L_{0}+E-\eta_{i, j_{2}}-\sum_{j=j_{2}}^{j_{i}-1}\left(\left(k_{i, j}-k_{i, j_{2}}\right)^{*} \rho_{i, j}\right) \mu_{i, j} .
$$

defined in the covering space $\bar{\pi}: \mathbb{R} \times \mathbb{T} \rightarrow \mathbb{T}^{2}$ in the coordinates $M_{3} x$.

Let $\gamma$ be a curve starting from the point $x_{i, 1}$ passing successively through the points $x_{i, j_{1}}, x_{i, j_{2}}$ and arriving at the point $x_{i, j_{i}}$ such that

1. $x_{i, j_{1}} \in \cup I_{c_{i, j_{1}}, 0, \ell}$ and $x_{i, j_{2}} \in \cup I_{c_{i, j_{2}}, 0, \ell}$

2. to the covering space $\mathbb{R} \times \mathbb{T}$ in the original coordinates, the lift of the segment joining $x_{i, 1}$ to $x_{i, j_{1}}$ is a curve minimizing the action of $\tilde{L}_{i, 1}$ along any curve connecting $\bar{x}_{i, 1}$ to $\bar{x}_{i, j_{1}}$, where $\bar{\pi} \bar{x}_{i, \imath}=x_{i, \imath}$ for $\imath=1, j_{1}$, $\bar{x}_{i, 1,1}<-k_{i, 1}$ and $\bar{x}_{i, j_{1}, 1}>k_{i, j_{1}}$ if we write $\bar{x}_{i, l}=\left(\bar{x}_{i, l, 1}, \bar{x}_{i, l, 2}\right)$ for $\imath=1, j_{1}$

3. to the covering space $\mathbb{R} \times \mathbb{T}$ in the coordinates $M_{2} x$, the lift of the segment joining $x_{i, j_{1}}$ to $x_{i, j_{2}}$ is a curve minimizing the action of $\tilde{L}_{i, 2}$ along any curve connecting $\bar{x}_{i, j_{1}}$ to $\bar{x}_{i, j_{2}}$, where $\bar{\pi} \bar{x}_{i, \imath}=x_{i, \imath}$ for $\imath=j_{1}, j_{2}$, $\bar{x}_{i, j_{1}, 1}<-k_{i, j_{1}}$ and $\bar{x}_{i, j_{2}, 1}>k_{i, j_{2}}$; 
4. to the covering space $\mathbb{R} \times \mathbb{T}$ in the coordinates $M_{3} x$, the lift of the segment joining $x_{i, j_{2}}$ to $x_{i, j_{i}}$ is a curve minimizing the action of $\tilde{L}_{i, 3}$ along any curve connecting $\bar{x}_{i, j_{2}}$ to $\bar{x}_{i, j_{i}}$, where $\bar{\pi} \bar{x}_{i, \imath}=x_{i, \imath}$, for $\imath=j_{2}, j_{i}$, $\bar{x}_{i, j_{2}, 1}<-k_{i, j_{2}}$ and $\bar{x}_{i, j_{i}, 1}>k_{i, j_{i}}$;

5. for $m=1,2$, when $x_{i, j_{m}}$ ranges over the set $\cup I_{c_{i, j_{m}}, 0, \ell}$, it is required that the corresponding $\bar{x}_{i, j_{m}}$ varies in the connected set $\Sigma_{0}^{n}, \bar{\Sigma}_{0}^{o}$ respectively.

Finally, we consider the quantity

$$
\begin{aligned}
A_{i}\left(x_{i, 1}, x_{i, j_{i}}\right) & =\min _{\substack{x_{i, j_{1}} \in \cup I_{c_{i, j_{1}, 0, \ell}} \\
x_{i, j_{2}} \in \cup I_{c_{i, j_{2}}, 0, \ell}}}\left\{A_{\tilde{L}_{i, 1}}\left(\bar{\gamma}_{i, 1}\left(\cdot, \bar{x}_{i, 1}, \bar{x}_{i, j_{1}}\right)\right)\right. \\
& \left.+A_{\tilde{L}_{i, 2}}\left(\bar{\gamma}_{i, 2}\left(\cdot, \bar{x}_{i, j_{1}}, \bar{x}_{i, j_{2}}\right)\right)+A_{\tilde{L}_{i, 3}}\left(\bar{\gamma}_{i, 3}\left(\cdot, \bar{x}_{i, j_{2}}, \bar{x}_{i, j_{i}}\right)\right)\right\}
\end{aligned}
$$

and denote by $\gamma_{i}\left(x_{i, 1}, x_{i, j_{i}}\right)$ the minimal curve. Clearly, the minimum is reached in the interior of $\cup I_{c_{i, j_{1}}, 0, \ell}$ and $\cup I_{c_{i, j_{2}}, 0, \ell}$ guaranteed by Lemma 6.1 if both $k_{i, j_{1}}-k_{i, j_{1}-1}$ and $k_{i, j_{2}}-k_{i, j_{2}-1}$ are sufficiently large, and it follows that the curve is smooth at both $x_{i, j_{1}}$ and $x_{i, j_{2}}$. The curve $\gamma_{i}\left(x_{i, 1}, x_{i, j_{i}}\right)$ obviously solves the Euler-Lagrange equation, it generates an orbit visiting $\delta_{i}$-neighborhood of $\tilde{\mathcal{A}}\left(c_{i, j}\right)$ for all $j=1,2, \cdots, j_{i}$

Proof of Theorem 1.3. For a sequence of $\delta_{1}>\delta_{2}>\cdots>\delta_{i}$, we consider the quantity

$$
A_{1, i}\left(x_{1,1}, x_{i, j_{i}}\right)=\min \sum_{l=1}^{i} A_{l}\left(x_{l, 1}, x_{l, j_{l}}\right),
$$

where the minimum is taken over the set $\left\{x_{l, j_{l}}=x_{l+1,1} \in \cup I_{c_{l+1,1}, 0, \ell}: l=\right.$ $1,2, \cdots, i\}$. Let $\gamma_{1, i}$ be the minimal curve, it is smooth at each point $x_{l, 1}$ for $l=2,3, \cdots, i-1$ provided $k_{l, j_{l}}-k_{i, j_{l}-1}$ is sufficiently large. By setting $k_{i, j}-k_{i, j-1} \rightarrow \infty$ as $i \rightarrow \infty$, the orbit generated by the minimal curve will visit the $\delta_{i}$-neighborhood of each $\tilde{\mathcal{A}}\left(c_{i, j}\right)$ with $j=1,2, \cdots, j_{i}$. By a timetranslation, we assume the curve $\gamma_{1, i}$ is defined on $t \in\left[0, T_{i}\right]$. Apparently, $T_{i} \rightarrow \infty$ as $i \rightarrow \infty$. Let $i \rightarrow \infty, \delta_{i} \rightarrow 0$, the curves $\left\{\gamma_{1, i}\right\}$ of $A_{1, i}\left(x_{1,1}, x_{i, j_{i}}\right)$ approaches a curve $\mathbb{R}^{+} \rightarrow \mathbb{T}^{2}$ which generates an orbit which is obviously transitive for $\cup_{c \in \alpha_{0}^{-1}(E)} \tilde{\mathcal{A}}(c)$.

By the proof, this theorem holds for suitably small $E>\min \alpha_{0}$. It is interesting to ask whether it holds for larger $E$. Apparently, it is not true if $E>0$ is sufficiently large. Indeed, by rescaling the time $t \rightarrow \sqrt{\lambda} t$, the Lagrangian is equivalent to

$$
L_{0, \lambda}=\frac{1}{2}\langle A \dot{x}, \dot{x}\rangle+\frac{1}{\lambda} V(x) .
$$


It reduces the energy from $E$ to $E / \lambda$. When $\lambda \rightarrow \infty$ it approaches an integrable one. For sufficiently large $\lambda>0$, there exist a lot of KAM invariant tori which block the transitivity.

Acknowledgement. The referee's comments are greatly appreciated, without which the article would not be in the present form. In particular, it is the referee's suggestion that the author add Theorem 1.3 to this version.

The main content of this paper is from the preprint [C13] which seems quite long. The author splits it into several parts with modifications, this article is one of them. The author is greatly appreciated for the discussion with Jinxin Xue, he also thanks Min Zhou for her patience to check the details. The author also thanks IMS at The Chinese University of Hong Kong for its hospitality, where the revised version was finished.

\section{References}

[A64] Arnold V. I., Instability of dynamical systems with many degrees of freedom, Sov. Math. Dokl., 5 (1964), 581-585; translation from Dokl. Akad. Nauk SSSR, 156 (1964), 9-12. MR0163026

[A66] Arnold V. I., The stability problem and ergodic properties for classical dynamical systems. Proceedings of International Congress of Mathematicians, Moscow (1966) 5-11, in V. I. Arnold-Collected Works. Springer Berlin Heidelberg (2014) 107-113. MR2640495

[AABZ] Arcostanzo M., Arnaud M., Bolle P. \& Zavidovique M., Tonelli Hamiltonians without conjugate points and $C^{0}$ integrability, Math. Z. 280 (2015) 165-194. MR3343902

[B00] Bernard P., Homoclinic orbit to invariant sets of quasi-integrable exact maps, Ergod. Theory Dynam. Syst. 20 (2000) 1583-1601. MR1804946

[B02] Bernard P., Connecting orbits of time dependent Lagrangian systems, Ann. Inst. Fourier, Grenoble 52 (2002) 1533-1568. MR1935556

[B07] Bernard P., Symplectic aspects of Mather theory, Duke Math. J. 136 (2007) 401-420. MR2309170

[B08] Bernard P., The dynamics of pseudographs in convex Hamiltonian systems, J. Amer. Math. Soc. 21 (2008) 615-669. MR2393423

[B10] Bernard P., Large normally hyperbolic cylinders in a priori stable Hamiltonian systems. Ann. H. Poincaré 11 (2010) 929-942. MR2736527 
[BC] Bernard P. \& Contreras G., A generic property of families of Lagrange systems, Annals of Math. 167 (2008) 1099-1108. MR2415395

[Bir] Birkhoff G. D., Dynamical Systems, Colloquium Publications of AMS, 9 (1927). MR0209095

[BI] Burago D. \& Ivanov S., Riemannian tori without conjugate point are flat, GAFA 4 (1994) 259-269. MR1274115

[CC] Cannarsa P. \& Cheng W., Homoclinic orbits and critical points of barrier functions, Nonlinearity 28 (2015) 1823-1840. MR3350611

[C11] Cheng C.-Q., Non-existence of KAM torus, Acta Math. Sinica, 27 (2011) 397-404. MR2754043

[C13] Cheng C.-Q., Arnold diffusion in nearly integrable Hamiltonian systems, arXiv:1207.4016v2 (2013).

[C15] Cheng C.-Q., Uniform hyperbolicity of invariant cylinder, arXiv 1510.08754 (2015), to appear in J. Differential Geometry.

[CY1] Cheng C.-Q. \& Yan J., Existence of diffusion orbits in a priori unstable Hamiltonian systems, J. Differential Geometry, 67 (2004) 457-517. MR2153027

[CY2] Cheng C.-Q. \& Yan J., Arnold diffusion in Hamiltonian Systems: a priori Unstable Case, J. Differential Geometry, 82 (2009) 229-277. MR2520793

[CX] Cheng C.-Q. \& Xue J., Arnold diffusion in nearly integrable Hamiltonian systems with arbitrary degrees of freedom, arXiv:1503.04153 (2015).

[CZ1] Cheng C.-Q. \& Zhou M., Non-degeneracy of extremal points in multi-dimensional space, Science China Math. 58 (2015) 22552260. MR3426129

[CZ2] Cheng C.-Q. \& Zhou M., Global normally hyperbolic cylinders in Lagrangian systems, Math. Res. Lett. 23 (2016) 685-705. MR3533191

[DLS06] Delshams A., de la Llave R. \& Seara T. M., A geometric mechanism for diffusion in Hamiltonian systems overcoming the large gap problem: heuristics and rigorous verification on a model. $\mathrm{Mem}$. Amer. Math. Soc 179 (2006) 844. MR2184276 
[DLS08] Delshams A., de la Llave R. \& Seara T. M., Geometric properties of the scattering map of a normally hyperbolic invariant manifold. Advances in Mathematics 217 (2008) 1096-1153. MR2383896

[El] Eliasson H., Biasymptotic solution of perturbed integrable Hamiltonian systems, Bol. Soc. Brasil. Mat. (N.S.) 25 (1994) 57-76. MR1274762

[Fa] Fathi A., Weak KAM Theorem in Lagrangian Dynamics, preprint (7-th preliminary version) (2005).

[KZ] Kaloshin V. \& Zhang K., A strong form of Arnold diffusion for two and a half degrees of freedom. arXiv:1212.1150v2 (2013).

[LC] Li X. \& Cheng C.-Q., Connecting orbits of autonomous Lagrangian systems, Nonlinearity 23 (2009) 119-141. MR2576377

[Lo] Lochak P., Canonical perturbation theory via simultaneous approximation, Russian Math. Surveys 47 (1992) 57-133. MR1209145

[Man] Mañé R., Generic properties and problems of minimizing measures of Lagrangian systems, Nonlinearity 9 (1996) 273-310. MR1384478

[M91] Mather J., Action minimizing invariant measures for positive definite Lagrangian systems, Math. Z., 207(2) (1991) 169-207. MR1109661

[M93] Mather J., Variational construction of connecting orbits, Ann. Inst. Fourier (Grenoble), 43(5) (1993) 1349-1386. MR1275203

[M03] Mather J., Arnold diffusion, I: Announcement of results, J. Mathematical Sciences, 124(5) (2004) 5275-5289.(Russian translation in Sovrem. Mat. Fundam. Napravl, 2 (2003) 116-130). MR2129140

[M11] Mather J., Shortest curve associated to a degenerate Jacobi metric on $\mathbb{T}^{2}$, Progress in Variational Methods, Nankai Ser. Pure Appl. Math. Theoret. Phys, 7 (2011) 126-168. MR2766806

[Mar] Marco J. P., Generic hyperbolic properties of classical systems on the torus $\mathbb{T}^{2}$, preprint (2013).

[Mas] Massart D., On Aubry sets and Mather's action functional, Israel J. Math. 134 (2003) 157-171. MR1972178

[MS] Massart D. \& Sorrentino A., Differentiability of Mather's average action and integrability on closed surfaces, Nonlinearity 24 (2011) 1777-1793. MR2793898 
[Mo] Moser J. K., On the volume elements on a manifold, Trans. Amer. Math. Soc. 120 (1965) 286-294. MR0182927

[Ox] Oxtoby J. C., Measure and category: A survey of the analogies between topological and measure spaces. Vol. 2. Springer Science 63 Business Media, 2013. MR0584443

[Tr1] Treschev D., The mechanism of destruction of resonant tori of Hamiltonian systems, Math. USSR Sbornik 68 (1991) 181-203. MR1025685

[Tr2] Treschev D., Evolution of slow variables in a priori unstable Hamiltonian systems, Nonlinearity, 17 (2004) 1803-1841. MR2086152

[Zhe] Zheng Y. \& Cheng C.-Q., Homoclinic orbits of positive definite Lagrangian systems, J. Diff. Eqns. 229 (2006) 297-316. MR2265629

[Zm1] Zhou M., Hölder regularity of weak KAM solutions in a priori unstable systems, Math. Res. Lett. 18 (2011) 75-92. MR2770583

[Zm2] Zhou M., Infinity of minimal homoclinic orbits, Nonlinearity, 24 (2011) 931-939. MR2772629

[Zm3] Zhou M., Non-degeneracy of Extremal Points, Chinese Annals of Math. B, 36 (2015) 45-50. MR3285679

Chong-Qing Cheng

DEPARTMENT OF MATHEMATICS

NANJING UNIVERSITY

NANJING 210093

CHINA

E-mail address: chengcq@nju.edu.cn

Received September 28, 2015 